\title{
Emergent Memory and Kinetic Hysteresis in Strongly Driven Networks
}

\author{
David Hartich $\odot^{*}$ and Aljaž Godec $\odot^{\dagger}$ \\ Mathematical bioPhysics Group, Max Planck Institute for Biophysical Chemistry, \\ 37077 Göttingen, Germany
}

(Received 7 April 2021; revised 20 August 2021; accepted 4 October 2021; published 8 December 2021)

\begin{abstract}
Stochastic network dynamics are typically assumed to be memoryless. Involving prolonged dwells interrupted by instantaneous transitions between nodes, such Markov networks stand as a coarse-graining paradigm for chemical reactions, gene expression, molecular machines, spreading of diseases, protein dynamics, diffusion in energy landscapes, epigenetics, and many others. However, as soon as transitions cease to be negligibly short, as often observed in experiments, the dynamics develops a memory. That is, state changes depend not only on the present state but also on the past. Here, we establish the first thermodynamically consistent-dissipation-preserving-mapping of continuous dynamics onto a network, which reveals ingrained dynamical symmetries and an unforeseen kinetic hysteresis. These symmetries impose three independent sources of fluctuations in state-to-state kinetics that determine the "flavor of memory." The hysteresis between the forward- or backward-in-time coarse graining of continuous trajectories implies a new paradigm for the thermodynamics of active molecular processes in the presence of memory, that is, beyond the assumption of local detailed balance. Our results provide a new understanding of fluctuations in the operation of molecular machines as well as catch bonds involved in cellular adhesion.
\end{abstract}

DOI: $10.1103 /$ PhysRevX.11.041047

Subject Areas: Biological Physics, Complex Systems Statistical Physics

\section{INTRODUCTION}

In the presence of a timescale separation, the coarse graining of continuous-space dynamics to transitions on a network yields memoryless Markovian kinetics. Such Markov networks are routinely used for the description of chemical reactions [1-3], gene expression [4,5], molecular machines [6], spreading of diseases [7], protein dynamics [8-12], diffusion in energy landscapes [13], epigenetics [14], and many others. Markov networks with only a few discrete states are useful for the modeling of a physical systems at large times in, for example, molecular machines [6] and proteins [8-12]. One inherent feature of memoryless dynamics is that the waiting time between consecutive state changes is exponentially distributed [15] as captured, e.g., by the Gillespie algorithm $[1,12]$.

To highlight how memoryless state-to-state transitions arise microscopically, we depict in Fig. 1(a) a realization of a continuous-space diffusion in a double-well potential as a

\footnotetext{
*david.hartich@mpibpc.mpg.de

†agodec@mpibpc.mpg.de
}

Published by the American Physical Society under the terms of the Creative Commons Attribution 4.0 International license. Further distribution of this work must maintain attribution to the author(s) and the published article's title, journal citation, and DOI. Open access publication funded by the Max Planck Society. function of time, which may represent, e.g., the extension of a protein molecule interconverting between two conformational states [17]. As soon as the barrier between the two wells is high enough, the system locally equilibrates within each well before transiting to the other, which renders the probability density of the exit time from either well [exits from well 2 are highlighted in Fig. 1(a)] to a good approximation exponentially distributed [see right panel in Fig. 1(a)]. More generally, two conditions must be satisfied for memoryless kinetics between metastable states to emerge [18]. To provide an understanding of these two conditions, it is useful to dissect each exit time into a transition period [19-21] (see black bars) and the rest that we call the dwell time (see orange bars). The first condition requires that the system, once it leaves any of the metastable states (e.g., state 2), quickly transits to the next state (i.e., the transition-path time is negligibly short) or rapidly returns to the initial state. In Fig. 1(a), the latter condition is visible as short excursions within the long "dwell-time" periods highlighted in orange. The second condition requires dwell periods to be long enough for the system to reach a local equilibrium in the initial well, which guarantees that any potentially hidden degree of freedom has also reached equilibrium. Memoryless kinetics thus involves the interplay of long dwells and short "instantaneous" transitions.

A two-state Markov-jump process-representing the minimum-to-minimum hopping in Fig. 1(a)-inherently 
(a) 1

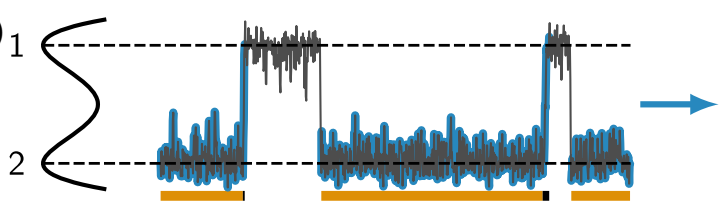

(b) 1

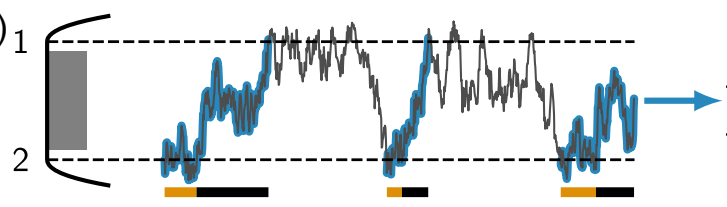

(c) 1

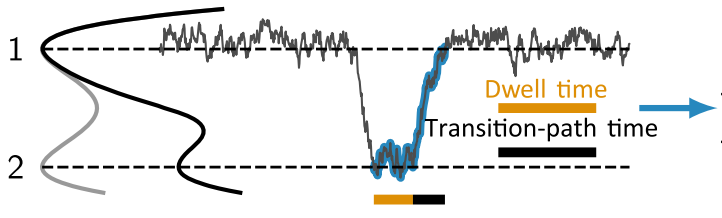

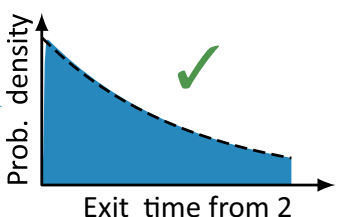
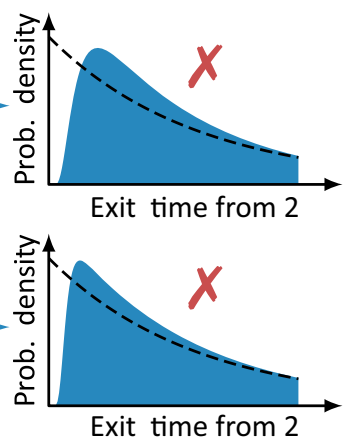

(d)

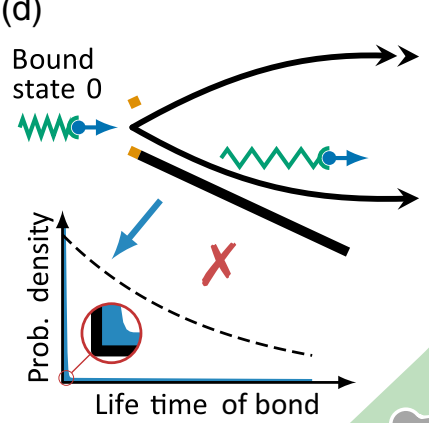

Pathway 1

MuK $\rightarrow$

Pathway 2

Whe $\rightarrow$

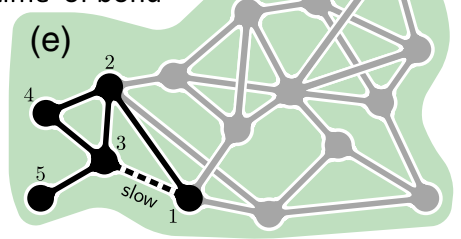

FIG. 1. Breakdown of Markovian and emergence of non-Markovian kinetics. (a) Left: diffusion in a double-well potential as a reduced model of the dynamics of a protein molecule transitioning between an unfolded (state 1) and a folded (state 2) conformation. Each exit event from state 2 to state 1 is highlighted in blue. Right: the histogram (shaded region) of the exit time from state 2 is well approximated by a memoryless single exponential decay (dashed line). Orange bars below the trajectory highlight dwell periods in the reduced state 2 , and black bars the duration of transitions from state 2 to state 1. (b) Left: diffusion in a potential with a diffusive barrier. Right: the histogram (shaded region) of the exit time from state 2 alongside a single exponential decay with the same mean exit time (dashed line) that, however, only poorly approximates the statistics of exit. (c) Double-well potential from panel (a) "tilted" by an additional pulling force that destabilizes the (folded) state 2. (d) Schematic of rupture pathways of a "catch bond" under force. The bond can rupture along two possible pathways: a fast pathway 1 (double arrow) and a slow pathway 2 that involves an intermediate conformational change. As before, the orange and black bars denote the dwell and transition periods, respectively. The probability density of the lifetime of the bond is shown below, whereby the probability densities depicted by the histogram (shaded region) and dashed line have the same mean. The stark disagreement between the two reflects that the rupture is not memoryless. (e) Schematics of a general network with a subnetwork with five states highlighted in black. Transitions between states 1 and 3 (dashed line) are assumed to be slow.

neglects a finite duration of transitions that can nowadays be probed in single-molecule fluorescence $[22,23]$ or force [24-26] spectroscopy experiments. Even when they are short, random transition times encode important information about the topological shape of the free-energy barriers [27]. This implies that a non-Markovian network theory that explicitly incorporates transition-path times, which is the main aim of this work, is desirable even in the presence of timescale separation.

More importantly, prolonged transition-path times [22-26,28-30] resulting, e.g., from spatial transport of molecules in chemical reactions under imperfect mixing [31,32], in the presence of a rugged energy landscape [33] as shown in Fig. 1(b), or external forces that destabilize local minima as in Fig. 1(c), are bound to cause "mild" violations of Markovianity. Moreover, dynamics in higher dimensions allows for the coexistence of parallel transition paths [27,29]. Parallel transition paths as depicted in Fig. 1(d) allow for the coexistence of fast and slow timescales that can cause "strong" violations of Markovianity manifested, e.g., as so-called catch bonds in cellular adhesion [34-36] which we discuss below in more detail.

The idea to account for nonexponential waiting-time distributions is not new and is, in fact, at the heart of the generalized master equation [37] (see also Refs. [38-42] with numerous applications that go beyond the scope of this article). While these models were constructed and applied phenomenologically to unravel interesting phenomena such as anomalous diffusion [43-45], their microscopic physical underpinning remains elusive. Moreover, the phenomenological construction of the generalized master equation [37-41] assures only that it is kinetically consistent, whereas it remains unclear under which conditions the resulting renewal dynamics is thermodynamically consistent. The latter turns out to be essential; we show that coarse graining and time reversal, in fact, do not commute giving rise to a phenomenon we coin kinetic hysteresis. This has important consequences for the quantification of dissipation.

To account for transitions with a finite duration in complex networks as shown in Fig. 1(e), here we develop a theory embodying an exact projection of continuous dynamics on a graph onto a network with discrete states. Diffusion on a graph arises quite generally from the averaging of fast degrees of freedom in Hamiltonian dynamics weakly coupled to a heat bath [46] (see also Ref. [47]) and includes both a position-dependent force and a position-dependent diffusion coefficient $[48,49]$. The coarse-grained dynamics evolves as jumps between the nodes. A state change occurs once the trajectory enters a 

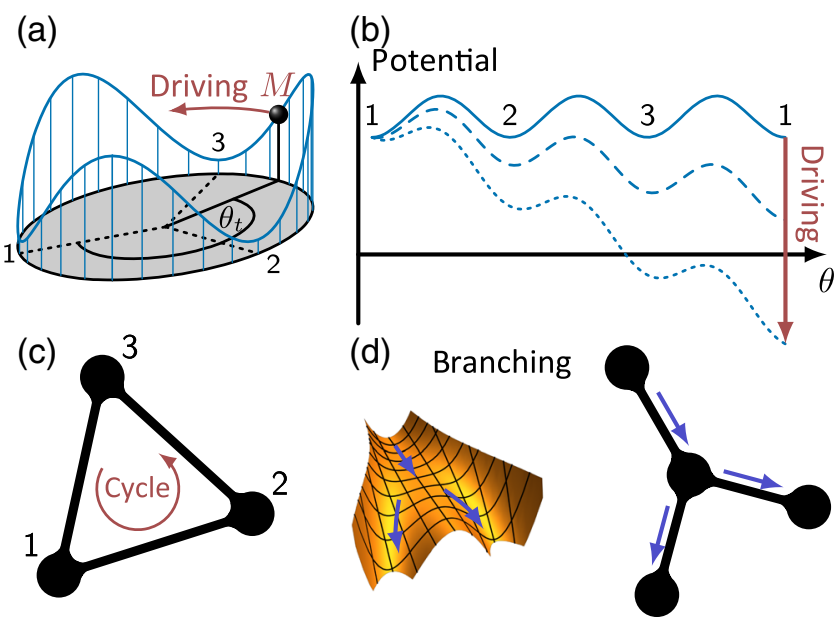

FIG. 2. Rationale and building blocks of coarse graining. (a)(c) Unicyclic (driven) systems and (d) branching point. (a) Rotation angle $\theta_{t}$ describing a system or observable driven by a nonequilibrium torque $M$ along a periodic potential with three metastable states. (b) The driving tilts the potential (dashed lines) which leads to work exchange along a cycle $1 \rightarrow 2 \rightarrow 3 \rightarrow 1$. (c) Network representation of (a) as in Fig. 1(e) (for corresponding trajectories, see Fig. 12). (d) Multiple cycles require branching points (blue arrows), for example, generated by a multidimensional force field with a local pitchfork bifurcation [14] (left panel depicts the corresponding local potential).

new node for the first time (see also Ref. [41], where the process is referred to as "milestoning").

Let us highlight two elementary "building blocks" of a network, which we call cycles and branching points (see Fig. 2), which are in fact not considered in Ref. [41]. The presence of cycles is required to enable a strongly driven system to constantly exchange (free) energy with the environment, thereby facilitating e.g., a biased transport. Consider an adenosine triphosphatase (ATPase) which has a threefold rotational symmetry [see Fig. 2(a)]. An external mechanical torque applied to the system causes a rotation [50], which in turn effectively gives rise to a nonconservative force field as illustrated in Fig. 2(b). In other words, upon closing a cycle $1 \rightarrow 2 \rightarrow 3 \rightarrow 1$ [see Fig. 2(c)], the system has made a $360^{\circ}$ turn while dissipating $M \times 360^{\circ}$ of free energy. We say that a network is strongly driven if the energy exchange substantially exceeds the thermal energy $k_{B} T$, which prevents the emergence of an equilibrium Boltzmann distribution. Note that the continuous dynamics on the graph shown in Fig. 2(c) inter alia takes into account possible intermediate metastable states observed, e.g., in Ref. [51].

Networks may have a genuinely multidimensional underlying topology that in turn allows for branching points as illustrated in Fig. 2(d). Branching points allow for the existence of multiple cycles; i.e., they account for multiple dissipative mechanisms. We consider the dynamics along the blue arrows in Fig. 2(d) to be effectively one dimensional, which assumes that the degrees perpendicular to the arrows are quickly relaxing [41,49]. More precisely, by considering graph networks as in Fig. 1(e), we assume that the dynamics is effectively concentrated along "tubes" connecting metastable states and/or branches. For example, complex topological free-energy landscapes can display such tubelike structures [52]. Later, we relax the assumption of paths concentrating along tubes and consider more general types of microscopic dynamics. In the following, we first outline how one can utilize dynamics on a graph to understand the emergence of non-Markovian dynamics on networks.

\section{A. Coarse graining}

We first consider a subgraph with five states highlighted in Fig. 1(e). A continuous trajectory on the graph is depicted in Fig. 3(a), where the time runs from bright to dark. Consider a gedanken experiment in which we record a "blinking" whenever the continuous trajectory enters a node that changes color upon each state change [Figs. 3(a) and 3(b)]. The time series of state changes arising from such a forward-in-time coarse graining is shown in Fig. 3(b) alongside recurrences, i.e., revisitations of nodes (see solid line and crosses, respectively). We measure the (local) joint probability density to exit state $i$ after a time $t$ and enter state $j, \wp_{j \mid i}^{\text {loc }}(t)$. Its marginal over time-the socalled splitting probability-defined as

$$
\phi_{j \mid i}^{\mathrm{loc}}=\int_{0}^{\infty} \wp_{j \mid i}^{\mathrm{loc}}(t) d t
$$

and is normalized according to $\sum_{j} \phi_{j \mid i}^{\text {loc }}=1$. Whenever $\wp_{j \mid i}^{\text {loc }}(t)$ deviates appreciably from an exponential function as in Fig. 3(c), the continuous trajectory does not locally equilibrate in $i$ before changing state to $j$, giving rise to memory in the coarse-grained dynamics. The reduced state change depicted in Fig. 3(c) forms a semi-Markov process [53,54] (see also Refs. [37,40]).

In the following, we explain the salient features of memory and the constraints that it imposes on the construction of thermodynamically consistent network dynamics.

\section{B. Summary of the main results}

As our first main result, we prove that the splitting probability obeys a reflection identity-the generalization of local detailed balance (see Sec. III):

$$
k_{B} T \ln \left(\phi_{j \mid i}^{\mathrm{loc}} / \phi_{i \mid j}^{\mathrm{loc}}\right)=g(i)-g(j)+\int_{i}^{j} \boldsymbol{F}(\boldsymbol{x}) \cdot d \boldsymbol{x},
$$

where $k_{B} T$ is the thermal energy, the quantity $g(i)-g(j)$ [defined in Eq. (15)] is strictly conservative, and the last term denotes the force integrated along the link from node $i$ to node $j$. Equation (2) connects the mesoscopic dynamics 
(a)

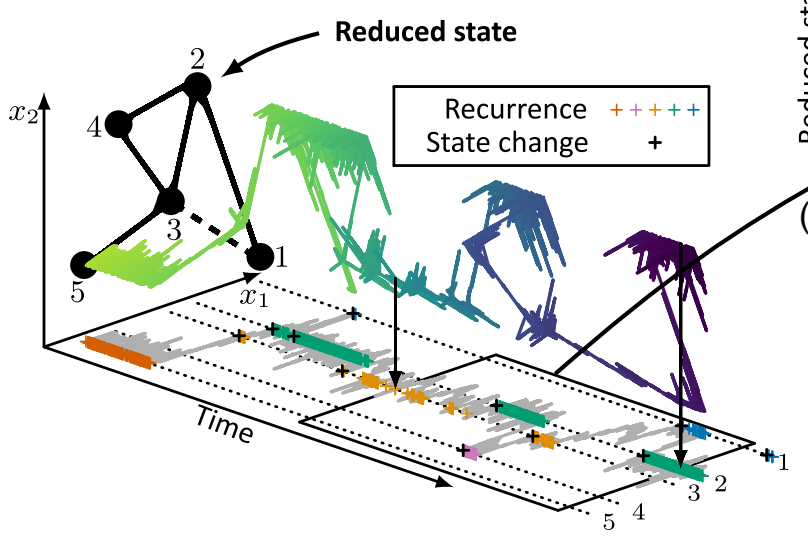

(b)

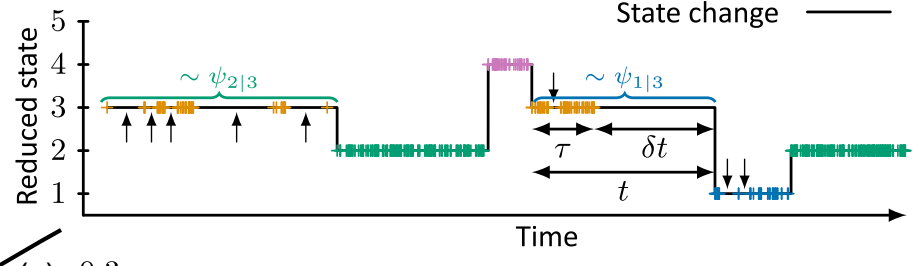

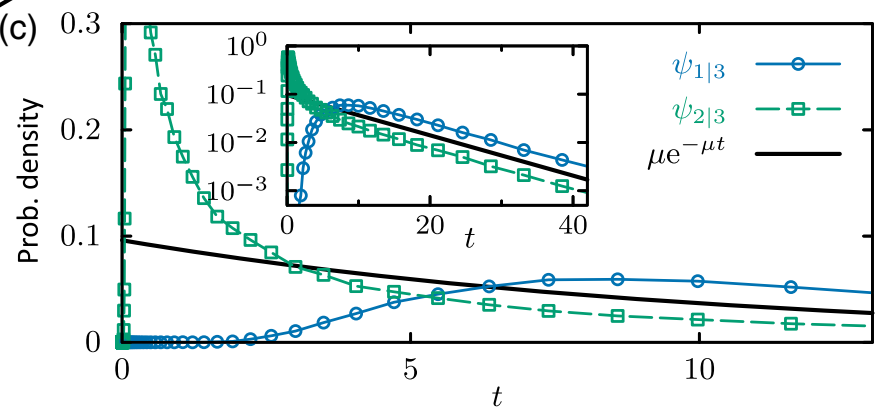

FIG. 3. Coarse-grained dynamics. (a) Projection of a trajectory of the full dynamics on the subgraph in Fig. 1(e) onto a plane (spanned by $x_{1} x_{2}$ ). Time runs from bright to dark. The network is represented by black lines with each reduced state $1, \ldots, 5$. A second projection onto the $x_{2}=0$ plane (gray line) reveals recurrences (colored crosses) and state changes (black crosses). (b) Coarse graining yields a time series of state changes (solid line); the time interval corresponds to the box in (a). One dwell interval " $\tau$ " and one transition-pathtime interval " $\delta t$ " are highlighted; their sum $t=\delta t+\tau$ is the local first-passage time from state $i$ (here $i=3$ ) to a neighboring state $j$ (here $j=1$ ). Long recurrence times are highlighted by vertical arrows. (c) Normalized probability density of local first-passage time $\psi_{j \mid i} \equiv \wp_{j \mid i}^{\text {loc }}(t) / \phi_{j \mid i}^{\text {loc }}$ [see also (b)] from $i=3$ to $j=1,2$. Details of the model are given in the Appendix E 1 .

in the presence of the memory embodied in $\phi_{j \mid i}^{\text {loc }}$ to the microscopic dissipative force $\boldsymbol{F}$ on the underlying graph. The force $\boldsymbol{F}$ may have a globally nonconservative contribution and alone encodes any violation of microscopic reversibility (i.e., detailed balance). The last term in Eq. (2) allows for an exchange of mechanical or (electro) chemical energy. Equation (2) ceases to hold if the coarse graining hides cycles [55,56], which we address in the dedicated Sec. VI A. Crucially, in both cases, the coarse graining into a discrete-state dynamics in the presence of memory must not commute with the time reversal, which gives rise to a phenomenon we refer to as kinetic hysteresis that is explained in Sec. III and illustrated in Fig. 6. In addition, we explain how the kinetic hysteresis resolves a puzzling conflict between two mutually contradicting views on irreversibility [53,54].

By means of the gedanken experiment in Fig. 3(a), we dissect each waiting time $t$ between two consecutive state changes as depicted in Fig. 3(b) into a dwell period $\tau$ spanning the time between the last state change until the last recurrence before the next state change, and the transition-path time $\delta t$, which is the time between the last recurrence and the next state change. The waiting time becomes the sum $t=\tau+\delta t$. This decomposition is in fact the key step toward understanding the emergence and manifestations of memory in network dynamics. As our second main result, we prove the statistical independence of local dwell and transition-path times emerging from an exact coarse graining of the underlying continuous dynamics (proof shown in Appendix B), i.e.,

$$
\psi_{j \mid i}(t) \equiv \frac{\wp_{j \mid i}^{\mathrm{loc}}(t)}{\phi_{j \mid i}^{\mathrm{loc}}}=\int_{0}^{t} \wp_{j \mid i}^{\mathrm{tr}}(t-\tau) \wp_{i}^{\mathrm{dwell}}(\tau) d \tau,
$$

where $\wp_{j \mid i}^{\mathrm{tr}}$ and $\wp_{i}^{\mathrm{dwell}}$ are the probability densities of the transition path and dwell time, respectively. Using Eq. (1), one finds that the independence holds if the new state $j$ is already known. Equation (3) embodies the following symmetries: (i) The dwell time is a state variable-it does not depend on the final state $j$ - and (ii) the transition-path time is reflection symmetric, $\wp_{i \mid j}^{\mathrm{tr}}(t)=\wp_{j \mid i}^{\mathrm{tr}}(t)$ (see also Ref. [57]). We prove both symmetries in Appendix B and illustrate symmetry (i) in Fig. 11 while symmetry (ii) is demonstrated in Fig. 13(c) as well as Table VII. Equation (3) is somewhat surprising since we find that slow transition kinetics, i.e., $\wp_{j \mid i}^{\mathrm{tr}}(t) \neq \delta(t)$, in fact (seemingly paradoxically) affect the statistics of dwell time $\wp_{i}^{\text {dwell }}$.

As our third and main practical result, we derive explicit formulas for the moments of transition-path time and dwell time, which are given in Eqs. (11)-(13). While moments of transition-path times are found to obey recursion integral formulas [58], we identify redundant integrals in the first two moments of the transition-path time that can be omitted and, interestingly, lead to an independent proof of the main finding in Ref. [27]. Moreover, we derive, for the first time, analytical formulas for the moments of the dwell time. The main consequence of this result is that transitions dictate the amplitude of fluctuations of the waiting time between any consecutive state change (see Sec. VA). We apply our main finding to two opposing scenarios. First, we show that large 
"super-Markovian" fluctuations in the waiting time (lifetime) observed in experiments with catch bonds [34,36] are unique signature parallel transitions between states that are unequally fast. Second, we show that symmetric stopping times of the entropy production (here called waiting time) in stationary driven systems [59-61] automatically imply equally fast transition times, which in turn yields "subMarkovian" dynamics, i.e., suppressed waiting-time fluctuations. Our theoretical results are directly applicable to the analysis of experimental time series.

\section{Relation to previous works and nomenclature}

Before we derive our results, let us briefly explain how the three main quantities, transition-path time $\delta t$, dwell time $\tau$, and waiting time $t$ [see Fig. 3(b)], relate to, and appeared in, previous works. The following paragraph summarizes the different terminologies used across the disciplines, which in turn helps us to identify and clarify the core of the conflict between Refs. [53,54].

First, the term waiting time [53] frequently appears under the terms (conditional) first-passage time [62,63], lifetime [63], stopping time [59], residence time, folding time [23], and even dwell time $[30,64,65]$ or cycle time [65]. Note that the waiting and dwell time coincide once the transition paths become instantaneous as in Refs. [64,65], and the inverse of the mean waiting time is also called Kramer's reaction velocity (rate) [66]. As illustrated in Fig. 1(d), the lifetime of a (catch) bond [34-36,67,68] represents a waiting time in the bound state. Second, the transitionpath time $[22,26,28,29,69]$ is sometimes also referred to as transition-event duration [58], translocation time [57], and occasionally, transition time [24,29] or transit time [26]. Third, the dwell time is also referred to as residence time [70] or "loops" [20,21]. The distinction among these three quantities is important for understanding the following puzzling conflict concerning the fundamental notion of "irreversibility" [53,54].

A trajectory obeying Hamilton's equations of motion is physically reversible, yet mathematically irreversible. That is, if we naively mathematically revert time along such a phase-space trajectory (i.e., we simply read it backward), the resulting trajectory will violate the equations of motion unless we take into account the well-known physical fact that momenta change sign under time reversal (that is, unless we "physically" revert time). In thermodynamics, this reversibility translates into the concept of detailed balance, which implies (at equilibrium) that the probability of any path is identical to the probability of the physically time-reverted path.

A network with a clear timescale separation, as depicted, e.g., in Fig. 1(a), that violates detailed balance may nevertheless "locally equilibrate" with all connected reservoirs prior to a transition to the consecutive state. In this case, the local detailed balance relation relates the forward and backward transition probabilities (or rates) to the entropy flux [71,72]. If states connected to different reservoirs are connected by links, the network can sustain probability fluxes between these states in a nonequilibrium steady state. This in turn breaks physical time-reversal symmetry [6]. Once a clear timescale separation, and thereby, a local equilibrium, cease to exist, the connection between the forward or backward path probabilities and the entropy flux becomes more subtle. In particular, the deep connection between the breaking of detailed balance and the breaking of (mathematical) time-reversal symmetry in semi-Markov processes [as depicted in Fig. 3(b)] [54] has been put into question in Ref. [53]. In fact, the example in Fig. 3 turns out to invalidate the main conclusion of Ref. [54]. In order to restore the view put forward in Ref. [54], we find that transition paths must be "odd" under time reversal, which gives rise to a phenomenon we call kinetic hysteresis. The kinetic hysteresis, in fact, restores the logical connection between the breaking of timereversal symmetry and the breaking of detailed balance.

\section{Structure of the article}

The remainder of this article is structured as follows: In Sec. II, we define diffusion on a graph depicted in Fig. 3 along with the precise coarse graining into digitized states. We discuss the limitations of the coarse graining, define transition-path time and dwell-time functionals, and explain their independence and symmetries that follow from Eq. (3). In Sec. II D, we present the main practical result. In Sec. III, we derive Eq. (2) and prove the thermodynamic consistency of the coarse graining. Surprisingly, we find in Sec. IV that the coarse graining must not commute with time reversal, which gives rise to a kinetic hysteresis. The kinetic hysteresis turns out to reconcile two contradicting views on the thermodynamics of irreversibility, namely, those between Refs. [53,54]. The central implications of Eqs. (10)-(13) are discussed in Sec. V, where we identify three fundamental sources of noise in the waiting time and explain the practical implications of deviations from Markovianity, in particular, the emergence of sub-Markovian fluctuations in driven periodic systems in Sec. V C and super-Markovian fluctuations in the presence of parallel transition paths in Sec. VB. Section VI provides a broader perspective of our results including the relation between the coarse graining and milestoning [18,63] (see Refs. [3,73] for a more elaborate exposé). We conclude in Sec. VII.

The derivations are rather involved and therefore relegated to a series of appendixes. Details about stochastic differential equations on a graph and their numerical implementation are given in Appendix A. The proof of Eq. (3) along with the entailed symmetries are shown in Appendix B. Our results are derived on the basis of a novel decomposition of paths shown in Appendix B 6, which represents a generalization of the renewal theorem [74]. The quite lengthy and tedious derivation of 
Eqs. (10)-(13) is explained in the Supplemental Material [75]. Equation (2) is proven in Appendix D, and the symmetries are tested in Appendix E.

\section{MODEL}

\section{A. Diffusion on a graph}

The full system's dynamics is assumed to evolve as piecewise continuous space-time Markovian diffusion on a graph as shown in Fig. 3(a) with potential (weak) discontinuities at the set of all nodes $i \in \Omega$. We denote all neighbor nodes of $i$ by $\mathcal{N}_{i} \subset \Omega$. For example, in Fig. 3(a), the set of neighboring states of state 2 are $\mathcal{N}_{2}=\{1,3,4\}$. At any time $t$ between the last passage by $i$ in the direction of $j$ at time $t_{\text {ini }}$ and the next visit of a node $j \in \mathcal{N}_{i}$ or the return to $i$ at time $t_{\text {fin }}$, i.e., $t_{\text {ini }}<t<t_{\text {fin }}$, the system is assumed to satisfy the anti-Itô (or Hänggi-Klimontovich $[76,77])$ Langevin equation

$$
\dot{x}_{t}=\beta D_{j \mid i}\left(x_{t}\right) F_{j \mid i}\left(x_{t}\right)+\sqrt{2 D_{j \mid i}\left(x_{t}\right)} \circledast \xi_{t},
$$

where $x_{t}$ [see Fig. 4(a)] denotes the instantaneous distance from node $i$ in the leg $i-j$ with $0<x_{t}<l_{j \mid i}, D_{j \mid i}(x)$ and $F_{j \mid i}(x)$ are the diffusion landscape and force field along the leg directed from $i$ to $j$, respectively, $\beta \equiv 1 /\left(k_{B} T\right)$, and $\xi_{t}$ is standard Gaussian white noise with zero mean, i.e., $\left\langle\xi_{t}\right\rangle=$ 0 and $\left\langle\xi_{t} \xi_{t^{\prime}}\right\rangle=\delta\left(t-t^{\prime}\right)$. The symbol "®” denotes the antiItô product (see Appendix A 1), and $l_{j \mid i}=l_{i \mid j}$ denotes the length of the path connecting nodes $i$ and $j$ (see Fig. 4).

Once a node $i$ is reached from within a leg, the consecutive leg is chosen, without loss of generality, randomly from the set of all neighbors $j^{\prime} \in \mathcal{N}_{i}$ with equal probability; i.e., the microstate $\boldsymbol{x}=\left(x_{t}, j, i\right)$ in Fig. 4(a) changes $(0, j, i) \rightarrow\left(0, j^{\prime}, i\right)$. Thereupon, the dynamics again evolves according to Eq. (4) until the next visit of a node. Similarly, as soon as the node $j$ is reached, the microstate changes to $\left(l_{j \mid i}, j, i\right) \rightarrow(0, k, j)$ with $k$ randomly chosen among the neighbors of node $j\left(k \in \mathcal{N}_{j}\right)$ with
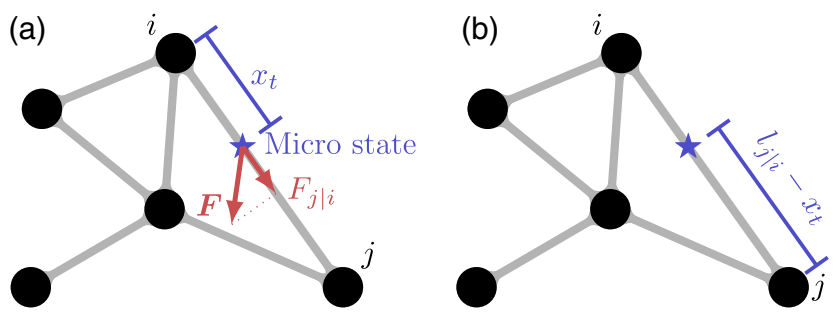

FIG. 4. Microstate versus network state on the path between nodes $i$ and $j$ separated by a distance $l_{j \mid i}$. (a) The microstate $\boldsymbol{x} \equiv\left(x_{t}, j, i\right)$ at distance $x_{t}$ from node $i$ in the direction toward node $j$ is driven by a force $\boldsymbol{F}$, where $F_{j \mid i}$ denotes the parallel component of the force. (b) The microstate measured by the distance $\tilde{x}_{t}=l_{j \mid i}-x_{t}$ from node $j$ is equivalent to the one from (a); i.e., $\boldsymbol{x}=\left(x_{t}, j, i\right)$ and $\tilde{\boldsymbol{x}}=\left(\tilde{x}_{t}, i, j\right)$ are equivalent. equal probability. This procedure fully specifies the full system's dynamics. In Appendix A 6, we translate the Langevin equation (4) into a Fokker-Planck equation, and in Appendix D 3 we explain in detail how one can account for discontinuities in the diffusion landscape and force field.

Three remarks are in order. First, in what follows we assume the Langevin equation (4) to determine the time evolution of the microstate along any link depicted by the gray lines in Fig. 4. It is shown that such a dynamics naturally emerges when possibly hidden degrees of freedom (perpendicular to the gray lines) are quickly relaxing [49]; that is, the full system's trajectories concentrate along "tubes." Strikingly, ignoring a possibly higher-dimensional embedding renders the diffusion coefficient spatially dependent [49] due to entropic effects. Diffusion on a graph also emerges from Hamiltonian dynamics weakly coupled to a heat bath [46].

Second, the Langevin equation (4) can globally violate detailed balance. Nevertheless, for any $i \in \Omega$ and $j \in \mathcal{N}_{i}$, the force translates along any link into the local potential

$$
U_{j \mid i}(x) \equiv-\int_{0}^{x} F_{j \mid i}(y) d y
$$

for $0<x<l_{i \mid j}$. The representation of the microstate by design entails a redundancy, meaning that $\boldsymbol{x}=\left(x_{t}, j, i\right)$ and $\tilde{\boldsymbol{x}}=\left(l_{j \mid i}-x_{t}, i, j\right)$ are the same microstate (see Fig. 4). This imposes the following reflection symmetries: $D_{i \mid j}(x)=$ $D_{j \mid i}\left(l_{j \mid i}-x\right)$ as well as $F_{i \mid j}(x)=-F_{j \mid i}\left(l_{j \mid i}-x\right)$, and hence, $U_{i \mid j}(x)=U_{j \mid i}\left(l_{j \mid i}-x\right)-U_{j \mid i}\left(l_{j \mid i}\right)$. If a global potential $\mathcal{U}$ exists, that is, $U_{j \mid i}\left(l_{j \mid i}\right)=\mathcal{U}_{j}-\mathcal{U}_{i}, \forall i, j \in \Omega$ with $j \in \mathcal{N}_{i}$ the dynamics is said to obey detailed balance. Conversely, if no such global potential exists, microscopic reversibility is said to be broken (see also Appendix D 1).

Third, we propagate Eq. (4) numerically using the Milstein scheme provided in Appendix A 3 whenever the diffusion coefficient is nonconstant " $D_{j \mid i}(x) \neq$ const." Otherwise, we use the stochastic Runge-Kutta scheme [78] described in Appendix A 4.

\section{B. Coarse graining to state changes on a network}

According to the gedanken experiment outlined in Fig. 3, the continuous trajectory is coarse grained into a time series of recurrences and state changes on a network. Consecutive visits of the continuous trajectory of the same node correspond to recurrences [see colored crosses in Figs. 3(a) and 3(b)], whereas transitions between distinct nodes yield state changes [see black crosses in Fig. 3(a) and line in Fig. 3(b)]. In between two consecutive state changes, the reduced network state remains in the initial state [see Fig. 3(b)]. This exactly specifies the coarse-grained trajectory on the network.

The dwell time $\tau$ corresponds to the sum of all consecutive recurrence times $t_{r}$ since the last state change. 
One dwell period is highlighted in Fig. 3(b). The transition time $\delta t$ corresponds to the time between the last recurrence and the instance of the state change. The local waiting time $t$ for a transition $i \rightarrow j$ is formally the sum of the dwell time and transition-path time $t=\delta t+\tau$ and corresponds to the time interval between two consecutive first entrances of nodes. Since the complete dynamics is stochastic, these quantities correspond to random variables. The joint probability density of a waiting time at $i$ and consecutive transition to $j$ corresponds to $\wp_{j \mid i}^{\text {loc }}(t)$, and the dwell and transition-path time are distributed according to $\wp_{i}^{\text {dwell }}(\tau)$ and $\wp_{j \mid i}^{\mathrm{tr}}(\delta t)$, respectively. Precise formal definitions of the waiting-, dwell-, and transition-path-time functionals entering Eq. (3) are given in the following subsection. In Appendix A 5, we explain the numerical evaluation of both dwell and transition-path time.

Let us briefly discuss the strengths and the limitations of the coarse graining. First, as we show Sec. III the coarse graining preserves the thermodynamic entropy production. Second, the coarse graining preserves node-to-node currents $[79,80]$ and its fluctuations, which are discussed in Sec. V C. Third, first passage functionals of the full microscopic model are preserved (see Appendix C 3). These are, for instance, crucial for catch-bond rupture experiments carried out in Refs. [34-36,67,68] (see Sec. V B). Fourth, the coarse graining retains vital information [27] encoded in transition-path times, which are the key to understanding the emergence of memory in the network kinetics that we discuss in Sec. V. However, the coarse graining has one shortcoming. Because of the aforementioned redundancy (see Fig. 4), the statistics of occupation time [81] (also known as "local time" or "empirical density" [82]) is not preserved. Occupationtime statistics on graphs were studied, for example, in Ref. [62]. We note that in the presence of a timescale separation, transitions become effectively instantaneous [see Fig. 1(a)], and in this limit, occupation times within the metastable regions are preserved, while concurrently the kinetics becomes memoryless (see Sec. III C).

\section{Definition of transition-path-time and dwell-time functionals}

Using the gedanken experiment depicted in Fig. 3(b), we define the dwell time $\tau$ as the time between the first "blinking" and the last blinking (last recurrence) of the same color (state), while the transition-path time denotes the time span between the last recurrence and the first following change of color. While the gedanken experiment allows for an intuitive definition of the dwell and transitionpath time, we now provide precise formal definitions that allow us to relate the gedanken experiment to existing definitions of transition-path times [19].

The transition path from node $i$ to node $j$ starts with the last recurrence to node $i$ and ends with the first visit of another node $j$ conditioned that $i$ has not been visited in between. Suppose that $x_{t}$ denotes the distance from node $i$ toward node $j$ satisfying the Langevin equation (4) between said nodes. Then the transition-path time is defined as the random variable [19]

$\delta t=\liminf _{y \rightarrow 0}\left\{t \mid x_{t}=l_{j \mid i} \wedge x_{0}=y \wedge\left(0<x_{\tau} \quad \forall 0 \leq \tau \leq t\right)\right\}$,

whose probability density function is denoted by $\gamma_{j \mid i}^{\mathrm{tr}}(\delta t)$. Note that an unsuccessful transition attempt terminates as soon as $x_{t}=0$, whereas a transition is successfully completed once $x_{t}=l_{j \mid i}$. Since transitions correspond to successful attempts only, we need to discard all unsuccessful attempts by introducing the transition Green's function $G_{j \mid i}^{\mathrm{tr}}$ defined as follows.

The probability density starting from $x_{0}=y$ to be found after time $t$ at distance $x$ from node $i$ in direction to node $j$, while never having either returned to state $i$ or reached state $j$, is denoted by $G_{j \mid i}^{\mathrm{tr}}(x, t \mid y)$. The probability density satisfies the initial condition $G_{j \mid i}^{\mathrm{tr}}(x, 0 \mid y)=$ $\delta(x-y)$. We translate the Langevin equation (4) into a Fokker-Planck equation [83] (see also Appendix A 6) $\partial_{t} G_{j \mid i}^{\mathrm{tr}}(x, t \mid y)=-\partial_{x} \hat{\mathcal{J}}_{j \mid i}^{F}(x) G_{j \mid i}^{\mathrm{tr}}(x, t \mid y)$, where $\hat{\mathcal{J}}_{j \mid i}^{\mathrm{F}}(x) \equiv$ $\beta D_{j \mid i}(x) F_{j \mid i}(x)-D_{j \mid i}(x) \partial_{x}$ is the current operator, and the boundary conditions are absorbing $G_{j \mid i}^{\mathrm{tr}}(0, t \mid y)=$ $G_{j \mid i}^{\mathrm{tr}}\left(l_{j \mid i}, t \mid y\right)=0$. The absorbing boundaries effectively terminate the process once either of the nodes $i$ or $j$ is reached. The transition-path time statistics are determined by taking the limit of successful trajectories in Eq. (6), that is, $y \rightarrow 0$ (starting from node $i$ ) and $x \rightarrow l_{j \mid i}$ (ending in node $j$ ). The corresponding probability density of transition-path time reads

$$
\wp_{j \mid i}^{\mathrm{tr}}(\delta t)=\lim _{y \rightarrow 0} \lim _{x \rightarrow l_{j \mid i}} \frac{\hat{\mathcal{J}}_{j \mid i}^{F}(x) G_{j \mid i}^{\mathrm{tr}}(x, \delta t \mid y)}{\int_{0}^{\infty} \hat{\mathcal{J}}_{j \mid i}^{F}(x) G_{j \mid i}^{\mathrm{tr}}(x, t \mid y) d t} .
$$

The dwell time is defined as follows: First, we define in node $i$ the state- $j$-dependent conditional first-passage time $t_{j}=\inf \left\{t \mid x_{0}=0 \wedge x_{t}=l_{j_{t} \mid i}\right\}$, where the index $j=j_{t}$ denotes the randomly chosen state following state $i$, which in turn can be used to define the dwell time as

$$
\tau=\sup _{t}\left\{t \mid x_{0}=0 \wedge x_{t}=0 \wedge t \leq t_{j}\right\} .
$$

The probability density of dwell time is denoted by $\wp_{j \mid i}^{\mathrm{dwell}}(\tau)$. We prove in Appendix B that the dwell time $\tau$ has in fact the same distribution for all final states $j$, which is manifested in the property $\wp_{j \mid i}^{\text {dwell }}(\tau)=\wp_{i}^{\text {dwell }}(\tau)$ symmetry (i) in Eq. (3). Moreover, we prove in Appendix B the independence of dwell and transition-path times, which 
allows us to represent the probability density of the conditional waiting time as a convolution as in Eq. (3).

\section{Main practical result}

A straightforward translation of Eq. (4) into a FokkerPlanck equation with appropriate boundary and internal continuity conditions allows us to obtain explicit results for the splitting probability and the statistics of dwell and transition-path time, which follow from some quite tedious algebra (see Supplemental Material [75]).

More precisely, in the Supplemental Material Sec. I in Ref. [75], we show that the path decomposition from Appendix C can be "inverted" to conveniently derive the statistics of waiting time $\wp_{j \mid i}^{\text {loc }}(t)$, which after insertion of the results derived in the Supplemental Material Sec. II in Ref. [75] — so-called unconditioned first-passage timesfinally yields the main practical result, Eqs. (10)-(13), as shown in the Supplemental Material Sec. III in Ref. [75].

For convenience, we introduce the following essential auxiliary integrals:

$$
I_{j \mid i}^{(k)} \equiv \int_{0}^{l_{j \mid i}} d y_{1} \int_{0}^{y_{1}} d y_{2}, \ldots, \int_{0}^{y_{k-1}} d y_{k} g_{j \mid i}^{(k)}
$$

where $g_{j \mid i}^{(k)}$ are depicted in Table I with the local potential $U_{j \mid i}$ defined in Eq. (5). In the following, we require only the first five integrals $I_{j \mid i}^{(k)}(k=1, \ldots, 5)$. Using the auxiliary integrals in Eq. (9), the splitting probabilities read

$$
\phi_{j \mid i}^{\mathrm{loc}}=\left(\sum_{k \in \mathcal{N}_{i}} I_{j \mid i}^{(1)} / I_{k \mid i}^{(1)}\right)^{-1},
$$

and the first two moments of the transition-path time become

$$
\langle\delta t\rangle_{j \mid i}^{\mathrm{tr}}=\frac{I_{j \mid i}^{(3)}}{I_{j \mid i}^{(1)}} \quad \text { and } \quad\left\langle\delta t^{2}\right\rangle_{j \mid i}^{\mathrm{tr}}=2\left(\langle\delta t\rangle_{j \mid i}^{\mathrm{tr}}\right)^{2}-2 \frac{I_{j \mid i}^{(5)}}{I_{j \mid i}^{(1)}},
$$

where the second moment is generally sub-Markovian, i.e., $\left\langle\delta t^{2}\right\rangle_{j \mid i}^{\mathrm{tr}} \leq 2\left(\langle\delta t\rangle_{j \mid i}^{\mathrm{tr}}\right)^{2}$. See also Ref. [27] for an alternative proof, where $\left\langle\delta t^{2}\right\rangle_{j \mid i}^{\mathrm{tr}} \leq 2\left(\langle\delta t\rangle_{j \mid i}^{\mathrm{tr}}\right)^{2}$ corresponds to a coefficient of variation being smaller than 1 . Some further extended algebra yields the first two moments of the average local dwell time

$$
\begin{aligned}
\langle\tau\rangle_{i}^{\mathrm{dwell}}= & \sum_{k \in \mathcal{N}_{i}} \phi_{k \mid i}^{\mathrm{loc}}\left[I_{k \mid i}^{(2)}-\langle\delta t\rangle_{k \mid i}^{\mathrm{tr}}\right] \\
\left\langle\tau^{2}\right\rangle_{i}^{\mathrm{dwell}}= & 2\left(\langle\tau\rangle_{i}^{\mathrm{dwell}}\right)^{2} \\
& +\sum_{k \in \mathcal{N}_{i}} \phi_{k \mid i}^{\mathrm{loc}}\left[2 I_{k \mid i}^{(2)}\langle\delta t\rangle_{k \mid i}^{\mathrm{tr}}-2 I_{k \mid i}^{(4)}-\left\langle\delta t^{2}\right\rangle_{k \mid i}^{\mathrm{tr}}\right]
\end{aligned}
$$

wherefrom follows the variance of the dwell time $\sigma_{\mathrm{dwell}, i}^{2}=\left\langle\tau^{2}\right\rangle_{i}^{\mathrm{dwell}}-\left(\langle\tau\rangle_{i}^{\mathrm{dwell}}\right)^{2}$. The independence of dwell and transition-path times in Eq. (3) immediately yields the binomial sum for the $n$th moment of the local first-passage time

$$
\left\langle t^{n}\right\rangle_{j \mid i}^{\mathrm{loc}}=\sum_{l=0}^{n}\left(\begin{array}{l}
n \\
l
\end{array}\right)\left\langle\delta t^{l}\right\rangle_{j \mid i}^{\mathrm{loc}}\left\langle\tau^{n-l}\right\rangle_{i}^{\mathrm{loc}}
$$

where the forward or backward symmetry implies $\left\langle\delta t^{l}\right\rangle_{j \mid i}^{\text {loc }}=\left\langle\delta t^{l}\right\rangle_{i \mid j}^{\text {loc }}$. The $n$th moment of the exit time is then simply given by $\left\langle t^{n}\right\rangle_{i}^{\text {exit }}=\sum_{k} \phi_{k \mid i}^{\text {loc }}\left\langle t^{n}\right\rangle_{k \mid i}^{\text {loc }}$ yielding the variance $\sigma_{\text {exit, } i}^{2}=\left\langle t^{2}\right\rangle_{i}^{\text {exit }}-\left(\langle t\rangle_{i}^{\text {exit }}\right)^{2}$. Note that $\langle t\rangle_{i}^{\text {exit }}$ is given in Eq. (S47) and $\left\langle t^{2}\right\rangle_{i}^{\text {exit }}$ can be found in Eq. (S50) in the Supplemental Material [75]. According to Eq. (13), the latter can be decomposed into three noise contributions $\sigma_{\mathrm{exit}, i}^{2}=\sigma_{\mathrm{dwell}, i}^{2}+\sigma_{\mathrm{tr}, \mathrm{int}, i}^{2}+\sigma_{\mathrm{tr}, \mathrm{ext}, i}^{2}$, where $\sigma_{\mathrm{dwell}, i}^{2}=\left\langle\tau^{2}\right\rangle_{i}^{\mathrm{dwell}}-$ $\left(\langle\tau\rangle_{i}^{\mathrm{dw} w e l l}\right)^{2}$, the intrinsic noise due to transition-path time is given by $\sigma_{\mathrm{tr}, \text { int }, i}^{2}=\sum_{k} \phi_{k \mid i}^{\mathrm{loc}}\left[\left\langle\delta t^{2}\right\rangle_{k \mid i}^{\mathrm{tr}}-\left(\langle\delta t\rangle_{k \mid i}^{\mathrm{tr}}\right)^{2}\right]$, and the extrinsic noise among different transition paths is given by $\sigma_{\mathrm{tr}, \mathrm{ext}, i}^{2}=\sum_{k} \phi_{k \mid i}^{\mathrm{loc}}\left(\langle\delta t\rangle_{k \mid i}^{\mathrm{tr}}\right)^{2}-\left(\sum_{k} \phi_{k \mid i}^{\mathrm{loc}}\langle\delta t\rangle_{k \mid i}^{\mathrm{tr}}\right)^{2}$.

Equations (10)-(13) are the main practical result of this paper. Notably, in Eq. (12), we determine for the first time the moments of dwell time. We emphasize that the results Eqs. (10)-(13) contain no redundant integrals that were eliminated in a quite tedious calculation shown in the Supplemental Material Secs. II.D and III in Ref. [75]. This final step is crucial for the derivation of the main result in

\begin{tabular}{|c|c|}
\hline$k$ & $g_{j \mid i}^{(k)}$ \\
\hline 1 & $e^{\beta U_{j \mid i}\left(y_{1}\right)} / D_{j \mid i}\left(y_{1}\right)$ \\
\hline 2 & $e^{\beta U_{j \mid i}\left(y_{1}\right)-\beta U_{j \mid i}\left(y_{2}\right)} / D_{j \mid i}\left(y_{1}\right)$ \\
\hline 3 & $e^{\beta U_{j \mid i}\left(y_{1}\right)-\beta U_{j \mid i}\left(y_{2}\right)+\beta U_{j \mid i}\left(y_{3}\right)} / D_{j \mid i}\left(y_{1}\right) D_{j \mid i}\left(y_{3}\right)$ \\
\hline 4 & $e^{\beta U_{j \mid i}\left(y_{1}\right)-\beta U_{j i \mid}\left(y_{2}\right)+\beta U_{j i \mid}\left(y_{3}\right)-\beta U_{j i \mid}\left(y_{4}\right)} / D_{j \mid i}\left(y_{1}\right) D_{j \mid i}\left(y_{3}\right)$ \\
\hline 5 & $e^{\beta U_{j i}\left(y_{1}\right)-\beta U_{j i}\left(y_{2}\right)+\beta U_{j i}\left(y_{3}\right)-\beta U_{j i}\left(y_{4}\right)+\beta U_{j \mid i}\left(y_{5}\right)} /\left[D_{j \mid i}\left(y_{1}\right) D_{j \mid i}\left(y_{3}\right)\right] D_{j \mid i}\left(y_{5}\right)$ \\
\hline
\end{tabular}
Sec. V. Moreover, due to the positivity of the surviving auxiliary integrals (9), Eq. (11) provides an independent proof of the main finding of Ref. [27]. If the network contains infinitely long legs $\left(l_{j-\mid i} \rightarrow \infty\right)$, the auxiliary integrals diverge. This leads to diverging moments of

TABLE I. Integrands entering Eq. (9) at a glance. 
dwell time that in turn may trigger interesting phenomena such as anomalous diffusion and "weak ergodicity breaking" [43-45,84]. Even in this case, the independence between transition and dwell time in Eq. (3) is expected to hold. We now address the thermodynamic consistency of the coarse graining.

\section{THERMODYNAMIC CONSISTENCY OF THE COARSE GRAINING}

\section{A. Splitting probability encodes thermodynamics}

In this section, we derive our first main result, Eq. (2), and explain its implications. In particular, we show that the coarse graining into the reduced-state dynamics preserves the dissipation (entropy production) of the underlying microscopic continuous dynamics in the presence of memory. The emergence of a kinetic hysteresis is discussed in the following section. Using Eq. (10), one obtains

$$
\ln \frac{\phi_{j \mid i}^{\mathrm{loc}}}{\phi_{i \mid j}^{\mathrm{loc}}}=\ln \left[\sum_{k \in \mathcal{N}_{j}} \frac{1}{I_{k \mid j}^{(1)}}\right]-\ln \left[\sum_{k \in \mathcal{N}_{i}} \frac{1}{I_{k \mid i}^{(1)}}\right]+\ln \frac{I_{i \mid j}^{(1)}}{I_{j \mid i}^{(1)}},
$$

where $I_{j \mid i}^{(1)}=\int_{0}^{l_{j \mid i}} D_{j \mid i}(x)^{-1} e^{\beta U_{j \mid i}(x)} d x \quad$ [cf. Eq. (9) and Table I]. To derive Eq. (2), we multiply Eq. (14) by the thermal energy and define

$$
g(\alpha) \equiv-k_{B} T \ln \left[\sum_{k \in \mathcal{N}_{\alpha}} \frac{1}{I_{k \mid \alpha}^{(1)}}\right],
$$

with $\alpha=i, j$. It remains to be shown that the last term in Eq. (14) is in fact the force integrated along the path starting from node $i$ and ending in node $j$ as in Eq. (2), which we prove in the following paragraph.

Using the auxiliary integrals from Eq. (9), we find

$$
\begin{aligned}
\ln \frac{I_{i \mid j}^{(1)}}{I_{j \mid i}^{(1)}} & =\ln \left[\frac{\int_{0}^{l_{j \mid i}} \frac{e^{\beta U_{i j}(x)}}{D_{i \mid j}(x)} d x}{\int_{0}^{l_{j \mid i}} \frac{e^{\beta U}}{D_{j \mid i}(x)} d x}\right]=\ln \left[\frac{\int_{0}^{l_{j \mid i}} \frac{e^{\beta U_{j \mid i}\left(l_{j \mid i}-x\right)-\beta U_{j \mid i}\left(l_{j \mid i}\right)}}{D_{j \mid i}\left(l_{j \mid i}-x\right)} d x}{\int_{0}^{l_{j \mid i}} \frac{e^{\beta U}}{D_{j \mid i}(x)} d x}\right] \\
& =-\beta U_{j \mid i}\left(l_{j \mid i}\right),
\end{aligned}
$$

where in the second step we use the symmetries $D_{i \mid j}(x)=$ $D_{j \mid i}\left(l_{j \mid i}-x\right)$ and $U_{i \mid j}(x)=U_{j \mid i}\left(l_{j \mid i}-x\right)-U_{j \mid i}\left(l_{j \mid i}\right)$, which are discussed in the paragraph following Eq. (5) in Sec. II A, and in the last step we use the fact that the integrals are identical up to the constant $e^{-\beta U_{j \mid i}\left(l_{j \mid i}\right)}$. We use $\beta=1 /\left(k_{B} T\right)$ and insert the definition of the local potential in Eq. (5) $U_{j \mid i}\left(l_{j \mid i}\right)=-\int_{0}^{l_{j \mid i}} F_{j \mid i}(x) d x$ into Eq. (16) to obtain

$$
k_{B} T \ln \frac{I_{i \mid j}^{(1)}}{I_{j \mid i}^{(1)}}=\int_{0}^{l_{j \mid i}} F_{j \mid i}(x) d x=\int_{i}^{j} \boldsymbol{F}(\boldsymbol{x}) \cdot d \boldsymbol{x},
$$

where in the last step we identify $F_{j \mid i}$ as the component of the force $\boldsymbol{F}$ along the link $i \rightarrow j$ [see Fig. 4(a)]. Inserting Eqs. (15) and (17) into Eq. (14) finally yields Eq. (2), which completes the proof of the first main result.

\section{B. Entropy production rate}

It is important to understand why Eq. (2) in fact encodes thermodynamic consistency, that is, why the coarse graining preserves the total entropy production rate of the underlying system at long times. During a long-time interval of length $t$, we observe $n_{j \mid i}(t)$ transitions from state $i$ to state $j$, which is a random number that in the limit of long times displays a non-negative stationary probability flow $\dot{n}_{j \mid i} \equiv \lim _{t \rightarrow \infty} n_{j \mid i}(t) / t \geq 0$. This stationary probability flow (see, e.g., Refs. [54,85]) can be calculated from $\dot{n}_{j \mid i}=\phi_{j \mid i}^{\text {loc }} \pi_{i} / \sum_{k}\langle t\rangle_{k}^{\text {exit }} \pi_{k}$, where $\pi$ is the unit eigenvector of the splitting matrix, i.e., $\pi_{j}=\sum_{i} \phi_{j \mid i}^{\text {loc }} \pi_{i}$. While detailed balance implies $\dot{n}_{j \mid i}-\dot{n}_{i \mid j}=0$, the violation of this equality, i.e., $\dot{n}_{j \mid i} \neq \dot{n}_{i \mid j}$, reflects a genuine breaking of detailed balance. Using the force field along the continuous graph, one can conveniently express the entropy production of the microscopic dynamics as

$$
\dot{S}^{\mathrm{SS}} \equiv \sum_{i, j} \dot{n}_{i j} \int_{i}^{j} d \boldsymbol{x} \cdot \boldsymbol{F} / T
$$

where $\int_{i}^{j} d \boldsymbol{x} \cdot \boldsymbol{F}$ is the dissipated "work" along a transition $i \rightarrow j$ at temperature $T$. Inserting Eq. (2) into Eq. (18) we obtain

$$
\begin{aligned}
\dot{S}^{\mathrm{SS}} & =k_{B} \sum_{i, j} \dot{n}_{j \mid i}\left[\frac{g(j)}{T}-\frac{g(i)}{T}+\ln \frac{\phi_{j \mid i}^{\mathrm{loc}}}{\phi_{i \mid j}^{\mathrm{loc}}}\right] \\
& =k_{B} \sum_{i, j} \dot{n}_{j \mid i} \ln \frac{\phi_{j \mid i}^{\mathrm{loc}}}{\phi_{i \mid j}^{\mathrm{loc}}}
\end{aligned}
$$

where in the final step we use Kirchhoff's law stating that all incoming flows and outgoing flows are conserved, i.e., $\sum_{j} \dot{n}_{i \mid j}=\sum_{j} \dot{n}_{j \mid i}$. We emphasize that Eq. (19) allows us to express the entropy production of the underlying microscopic dynamics in Eq. (18) solely in terms of the coarsegrained network dynamics $\left(\phi_{j \mid i}\right.$ and $\left.\dot{n}_{j \mid i}\right)$. This finding renders the coarse graining (see Fig. 3) thermodynamically consistent. Moreover, Eq. (19) explicitly does not require the underlying microscopic force field $\boldsymbol{F}$ entering the righthand side of Eq. (18) to be known.

A few additional remarks are in order. First, the coarse graining preserves the stationary entropy production rate since it does not hide cycles [55]. The preservation of cycles by the coarse graining is explicitly explained in Appendix D 1. Possible extensions to the theory including hidden cycles will be discussed in Sec. VI. In the presence of hidden cycles, Eq. (19) is expected to underestimate the 
entropy production rate. Second, in the limit of a timescale separation, Eq. (19) coincides with the entropy production in Markov networks [86], whereby Eq. (18) encapsulates the local detailed balance relation [6]. In the following subsection, we briefly address this limit, which arises in the presence of high local free-energy barriers that in turn yield memoryless kinetics.

\section{The peculiar limit of local detailed balance}

In the case of high local (free-) energy barriers corresponding to $B_{j \mid i} \rightarrow \infty$ and $B_{i \mid j} \rightarrow \infty$ in Fig. 5 for each pair $i, j$, the full microscopic trajectory locally equilibrates in each well prior to any transition. In this limit, the transition rate to jump from node $i$ to node $j$ becomes $w_{i \rightarrow j} \equiv \phi_{j \mid i}^{\text {loc }} /\langle t\rangle_{i}^{\text {exit }}$, and in turn Eq. (2) implies (see proof in Appendix D 2)

$$
k_{B} T \ln \frac{w_{i \rightarrow j}}{w_{j \rightarrow i}} \simeq \underbrace{\int_{i}^{j} \boldsymbol{F}(\boldsymbol{x}) \cdot d \boldsymbol{x}+\mathcal{U}_{j}-\mathcal{U}_{i}}_{\text {"work" along transition } i \rightarrow j}+\overbrace{\mathcal{F}_{i}-\mathcal{F}_{j}}^{\text {free-energy difference }},
$$

where $\mathcal{U}_{i}$ denotes the potential energy of node $i$, the first term on the right denotes the external free-energy input along the transition (i.e., "dissipated work"), and the free energy of state $i$ is defined by $\mathcal{F}_{i}=-k_{B} T \ln \mathcal{Z}_{i}$ with partition function $\mathcal{Z}_{i} \equiv \sum_{k \in \mathcal{N}_{i}} \int_{0}^{x_{k \mid i}^{*}} e^{-\beta\left[\mathcal{U}_{i}+U_{k \mid i}(x)\right]} d x$. The symbol " $\simeq$ " denotes asymptotic equality " $=$," here taken in the limit of high local (free-) energy barriers $\left(B_{j \mid i} \rightarrow \infty\right.$ and $B_{i \mid j} \rightarrow \infty$ ). Equation (20) reflects the so-called local

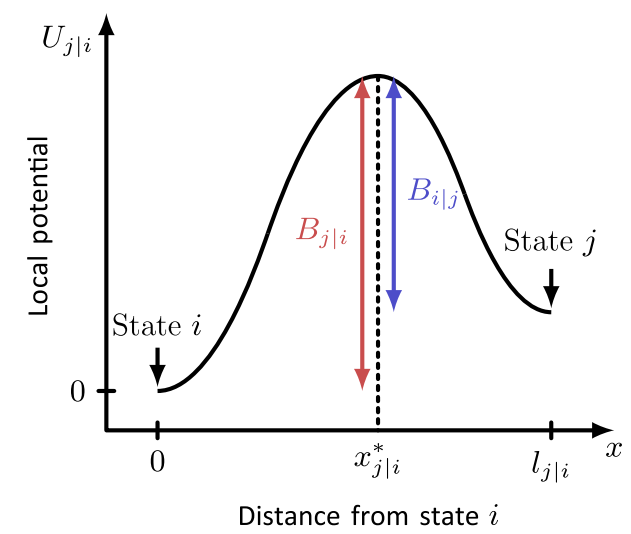

FIG. 5. Local potential with local equilibration. Local potential $U_{j \mid i}(x)=-\int_{0}^{x} F_{j \mid i}(y) d y$ between one pair of nodes $i$ and $j$. The two states are separated by a single maximum at $x_{j \mid i}^{*}$ of the local potential characterized by $F_{j \mid i}\left(x_{j \mid i}^{*}\right)=0$, while satisfying $F_{j \mid i}(x)<0$ if $x<x_{j \mid i}^{*}$ and $F_{j \mid i}(x)>0$ if $x>x_{j \mid i}^{*}$. Local equilibration occurs if the local free-energy barriers are high, meaning that $B_{j \mid i} \gg k_{B} T$ and $B_{i \mid j} \gg k_{B} T$. detailed balance [6] (or partial equilibrium [3]). When there is no work performed along the transition, local detailed balance also implies global detailed balance. Local detailed balance is violated as soon as a single barrier $B_{j \mid i}$ ceases to be high. Interestingly, local detailed balance [Eq. (20)] can be violated even in systems obeying detailed balance globally. In this case, the waiting-time distribution becomes nonexponential as in Fig. 3(c).

It is worth mentioning that the approximation (20) affects only the free-energy difference. This approximation still exactly satisfies

$$
\begin{aligned}
T \dot{S}^{S S} & =k_{B} T \sum_{i j} \dot{n}_{j \mid i} \ln \frac{w_{i \rightarrow j}}{w_{j \rightarrow i}} \\
& =\sum_{i j} \dot{n}_{j \mid i}\left[\int_{i}^{j} \boldsymbol{F}(\boldsymbol{x}) \cdot d \boldsymbol{x}+\mathcal{U}_{j}-\mathcal{U}_{i}+\mathcal{F}_{i}-\mathcal{F}_{j}\right] .
\end{aligned}
$$

The equality follows from Kirchhoff's law stating that incoming and outgoing currents balance each other $\sum_{j} \dot{n}_{j \mid i}=\sum_{j} \dot{n}_{i \mid j}$, as well as from Eqs. (18) and (19) with $w_{i \rightarrow j}=\phi_{j \mid i}^{\text {loc }} /\langle t\rangle_{i}^{\text {exit }}$.

\section{TIME REVERSAL AND KINETIC HYSTERESIS}

The dissipation in a system was found to be closely linked to the breaking of time-reversal symmetry (measured by the Kullback-Leibler divergence) in Hamiltonian systems under time-dependent driving [87,88], Markovian diffusion [89-91], and Markov-jump dynamics [92,93] to name but a few. These findings imply that a microscopic trajectory $\boldsymbol{\Gamma}_{\tau}=\boldsymbol{x}(t)_{0 \leq t \leq \tau}$ in a stationary ensemble of paths with measure $\mathcal{P}$ relates to the steady-state dissipation rate via

$$
\dot{S}_{\mathrm{KL}}=k_{B} \lim _{\tau \rightarrow \infty} \frac{1}{\tau}\left\langle\ln \frac{\mathcal{P}\left[\boldsymbol{\Gamma}_{\tau}\right]}{\mathcal{P}\left[\boldsymbol{\Gamma}_{\tau}^{R}\right]}\right\rangle,
$$

where $\boldsymbol{\Gamma}_{\tau}^{R}=\boldsymbol{x}(\tau-t)_{0 \leq t \leq \tau}$ is the time-reversed trajectory, and $\langle\cdots\rangle$ is the average over the forward path measure $\mathcal{P}\left[\boldsymbol{\Gamma}_{\tau}\right]$. Note that we consider overdamped dynamics; i.e., the microstate instantaneously "loses" momentum which is odd under time reversal (e.g., see Refs. $[87,88])$. In Fig. 3(a), the time-reversed trajectory $\boldsymbol{\Gamma}_{t}^{R}$ corresponds to the color-gradient line with time running from dark to bright. In fact, by design the entropy production rates in Eq. (22) coincide with the entropy production rate in Eq. (18), and therefore also with the one deduced from the coarsegrained trajectory Eq. (19); i.e., $\dot{S}_{\mathrm{KL}}^{\mathrm{SS}}=\dot{S}^{\mathrm{SS}}$ holds. Thus, the entropy production rate $\dot{S}^{S S}$ measures both the breaking of time-reversal symmetry of the underlying diffusive dynamics and the breaking of detailed balance. 
Two contradicting views have been put forward $[53,54]$ when addressing coarse-grained dynamics depicted in Fig. 3(b) that represents a semi-Markov chain. While Ref. [53] showed that the breaking of time reversibility does not imply breaking of detailed balance, Ref. [54] came to the exact opposite conclusion. We now show that this conflict in fact unravels a counterintuitive phenomenon.

Determining the breaking of time-reversal symmetry in a coarse-grained process according to Eq. (22) can in general be challenging. However, for a semi-Markov process one can elegantly determine the relative entropy rate in Eq. (22) from the waiting-time density $[54,85]$

$$
\dot{S}_{\mathrm{KL}}^{\mathrm{CG}}=\dot{S}^{\mathrm{SS}}+k_{B} \sum_{i, j, k} \phi_{k \mid j}^{\mathrm{loc}} \dot{n}_{j \mid i} D_{\mathrm{KL}}\left[\psi_{k \mid j}(t) \| \psi_{i \mid j}(t)\right],
$$

which follows immediately from the main result in Ref. [54] [see Eqs. (2)-(4) therein] along with the insertion of Eq. (19) and the definition of the Kullback-Leibler divergence $D_{\mathrm{KL}}[p(t) \| q(t)] \equiv \int_{0}^{\infty} p(t) \ln p(t) / q(t) d t \geq 0$. Equation (23) quantifies the mathematical time irreversibility of the coarse-grained process depicted in Fig. 3(b).

In contradiction to Ref. [53], the last term in Eq. (23) was believed to allow for the detection of "broken detailed balance in the absence of observable currents" [54]. Here we surprisingly find that the Kullback-Leibler divergence overestimates the entropy production, i.e., $\dot{S}_{\mathrm{KL}}^{\mathrm{CG}} \geq \dot{S}_{\mathrm{KL}}=\dot{S}^{S S}$. Notably, the process in Fig. 3(a), which is a manifestly equilibrium process with $\dot{S}^{S S}=0$, would paradoxically display a strictly positive rate $\dot{S}_{\mathrm{KL}}^{\mathrm{CG}}>0$. This follows immediately from the fact that the waiting-time densities in Fig. 3(c) are not equal. Thus, (coupled) anisotropic waiting-time distributions as in Fig. 3(c) are a signature of mathematical irreversibility [53], whereas they are in general not a signature of the breaking of detailed balance as apparently erroneously concluded in Ref. [54]. Interestingly, we do not find any technical mistake in the calculation in Ref. [54], yet our model provides a counterexample. How can we reconcile this?

It turns out that the coarse-grained trajectory depicted in Fig. 3(b) displays the following counterintuitive phenomenon. If we coarse grain the same trajectory backward in time, we discover, somewhat surprisingly, a kinetic hysteresis. That is, the time-reversed coarsened trajectory (see dotted gray line Fig. 6), where time is running from right to left, differs from the forward one. This hysteresis allows for a unique decomposition of each waiting time $t$ in any given node into a dwell time $\tau$-the interval in which the forward and time-reversed coarsened trajectory coincide-and a transition-path time $\delta t$ - the interval in which they differ (see Fig. 6).

To physically revert time, we must also physically revert the gedanken experiment of the state visits [see colored crosses in Figs. 6 and 3(b)]. Thus, each state visit at the end of a forward-in-time dwell period marks the first state

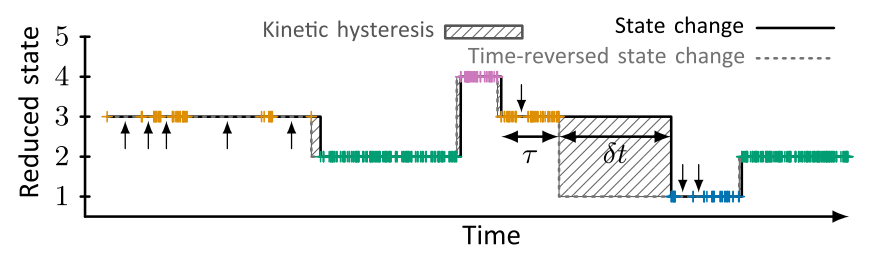

FIG. 6. Kinetic hysteresis. The coarse graining does not commute with time reversal. Transitions are "odd" under time reversal, while dwell periods are "even," i.e., dwell periods commute with time reversal. The state change is the same as in Fig. 3.

change in the time-reversed experiment. In other words, the time reversal must be carried out before the coarse graining, not after. This restores the connection between the breaking of physical time-reversal symmetry and violations of detailed balance; i.e., it reconciles the opposing views put forward in Refs. [53,54].

There is an analogy between transition paths and momenta, which explains the problem in Ref. [54]. If we were to reverse in time a trajectory in an equilibrium system (without changing the sign of momenta), we would obtain an unphysical time-reversed trajectory that can never be observed; i.e., Eq. (22) would yield a diverging entropy production rate at equilibrium. To avoid this unphysical result, one must take into account that momenta in fact change sign under time reversal $[87,88,92]$. Hence, we find that the transition paths, similar to momenta in underdamped systems $[87,88]$, in some sense are odd under time reversal, which gives rise to the kinetic hysteresis in Fig. 6 . In other words, the "coarse graining" and "time reversal" must not commute, which will lead to a paradigm shift in the understanding of time reversal in the presence of transition paths with a finite duration.

Some further remarks are in order. The thermodynamically inconsistent second term in Eq. (23) vanishes if the waiting-time distribution is decoupled from the state change [54] as studied in Refs. [94-96], which in fact follows from Eq. (3) in the limit of instantaneous transitionpath times $\wp_{j \mid i}^{\mathrm{tr}}(t)=\delta(t)$. That is, in this limit the waitingtime distribution $\psi_{j \mid i}(t)=\wp_{i}^{\text {dwell }}(t)$ does not depend on $j$. Second, our finding $\dot{S}_{\mathrm{KL}}^{\mathrm{CG}} \geq \dot{S}^{\mathrm{SS}}$ does not contradict Refs. $[88,97]$ since the path weight of the coarse-grained process is not a marginal path weight of the full one (see Sec. II B and Fig. 4). Third, until now, we considered the coarse graining into individual nodes (i.e., all cycles were preserved). It has been found that for certain network topologies, the lumping of nodes that hides cycles may lead to what is called a "second-order semi-Markov process" [54]. The kinetics in the presence of such "lumped" nodes is discussed in Sec. VIB. In this case, the connection between the entropy production rate and coarse-grained dynamics embodied in Eqs. (18) and (19) is expected to disappear. 


\section{THREE SOURCES OF FLUCTUATIONS}

\section{A. Transition noise dictates the amplitude of fluctuations}

Memory in state changes emerges locally as a result of long-recurrence and transition-path times. Long-recurrence times arise whenever the continuous trajectory becomes trapped in the legs of the subgraph without changing state [see vertical arrows in Fig. 3(b) or Fig. 6]. Long-transitionpath times are due to slow dynamics between a pair of adjacent nodes. Imagine that only one leg in Fig. 1(e), say, $3 \rightarrow 1$, displays slow or recurrent dynamics, e.g., because of slow diffusion and/or the absence of an energy barrier. Then, not only is $\psi_{1 / 3}$ clearly nonexponential [see blue line in Fig. 3(c)], but strikingly, also $\psi_{2 \mid 3}$ and all others become nonexponential [see green line in Fig. 3(c)]; the waitingtime distribution becomes "coupled" $[39,43,98]$ to the state change. Note that this problem cannot be solved within the framework of the generalized master equation [37] because the coupling has to be "put in by hand."

In order to understand the emergence these anisotropic local "waiting times," we dissect fluctuations of time required to exit state $i$ as $\wp_{i}^{\text {exit }}(t) \equiv \sum_{j} \wp_{j \mid i}^{\text {loc }}(t)$. The independence of dwell and transition-path times in Eq. (3) implies three independent contributions to fluctuations

$$
\sigma_{\text {exit }}^{2}=\sigma_{\text {dwell }}^{2}+\sigma_{\text {tr,int }}^{2}+\sigma_{\text {tr,ext }}^{2},
$$

where $\sigma^{2} \equiv\left\langle t^{2}\right\rangle-\langle t\rangle^{2}$ denotes the variance, and we further decompose fluctuations of transition-path time into intrinsic fluctuations along the respective legs of the subgraph $\sigma_{\mathrm{tr}, \text { int }}^{2}=\sum_{j} \phi_{j \mid i}^{\mathrm{loc}} \sigma_{\mathrm{tr}, j \mid i}^{2}$ and the extrinsic scatter of mean transition-path times among distinct legs $\sigma_{\mathrm{tr} \text { ext }}^{2} \equiv$ $\sum_{j} \phi_{j \mid i}^{\mathrm{loc}}\left(\langle\delta t\rangle_{j \mid i}^{\mathrm{tr}}\right)^{2}-\left(\sum_{j} \phi_{j \mid i}^{\mathrm{loc}}\langle\delta t\rangle_{j \mid i}^{\mathrm{tr}}\right)^{2}$. The three contributions in Eq. (24) are explained in Fig. 7(a) and given explicitly in Sec. II D.

When $\sigma_{\text {tr,ext }}^{2}$ vanishes, i.e., $\langle\delta t\rangle_{j \mid i}^{\mathrm{tr}}=\langle\delta t\rangle_{k \mid i}^{\mathrm{tr}}$ for all $j, k$ [see Fig. 7(a) left], the fluctuations of exit time are subMarkovian since $\sigma_{\text {exit }} \leq\langle t\rangle_{j}^{\text {exit }}$. In turn, super-Markovian fluctuations, that is, $\sigma_{\text {exit }} \geq\langle t\rangle^{\text {exit }}$, necessarily imply the existence of multiple exit pathways with distinct transitionpath times [see Fig. 7(a) right]. This proves that one can infer, in general, the existence of parallel transition pathways without actually resolving individual pathways, which is our third main result of this paper (for proof, see the last subsection in the Supplemental Material [75]). Below, we illustrate this main finding by means of two opposing examples.

\section{B. Super-Markovian exit dynamics reflect parallel unequally fast transition paths}

In a first demonstration of the practical implications of our results, we address the counterintuitive catch-bond phenomenon [35,67] depicted in Fig. 1(d) (see also Refs. [34,36]). A ligand bound to a receptor is pulled by a constant force $F$ until the bond ruptures (details about the model are given in Appendix E2). The time of rupture corresponds mathematically to the exit time from the bound state. A characteristic of catch bonds is that they rupture along two possible pathways. One pathway involves a conformational change of the receptor that prolongs the transition-path time. In turn, this gives rise to a nonmonotonic force dependence of the rupture time [see Fig. 7(c)]. That is, within a certain interval of $F$-the so-called catch-bond phase [34-36,68] — the bond counterintuitively survives longer if we pull stronger. The mean lifetime $\langle t\rangle^{\text {exit }}$ and its standard deviation $\sigma_{\text {exit }}$ reconstructed according to Refs. [35,67] are depicted in Fig. 7(c), where the lines denote exact results (see Sec. II D) and symbols are deduced from 500 simulated rupture events. A larger pulling force increases the likelihood of choosing the slow path [see black line in Fig. 7(b)] and in turn amplifies extrinsic noise [see shaded areas reflecting relative noise contributions in Fig. 7(b) as well as red symbols in Fig. 7 (a)]. The observed fluctuations are evidently superMarkovian, i.e., $\sigma_{\text {exit }} \geq\langle t\rangle^{\text {exit }}$, and therefore immediately imply the existence of at least two rupture pathways that are not equally fast $\sigma_{\text {trext }} \neq 0$. We show the decomposition of the lifetime of the bond into dwell and transition-path time along the individual pathways in Appendix E2 (see Fig. 11). If transition-path times can be measured explicitly, one can alternatively detect parallel paths by means of the coefficient of variation as explained in Ref. [27], which for the sake of completeness is shown in Appendix E2 e (see Fig. 10).

\section{Symmetry in transitions causes sub-Markovian exit dynamics}

We now consider the scenario where extrinsic transition noise vanishes, implying $\sigma_{\text {exit }} \leq\langle t\rangle^{\text {exit }}$. Particularly important examples are the steady-state operation of driven molecular machines and the more abstract "stopping times" of the thermodynamic entropy production [59-61]. We consider an ATPase operating under the influence of a nonequilibrium torque $M$, were $M=0$ refers to the torque at which the ATPase stalls [50]. The rotation of the ATPase evolves as diffusion in a periodic potential with period $2 \pi / 3$ reflecting the $120^{\circ}$ rotational symmetry of the motor, and a barrier height of $5 k_{B} T$ separating the well-defined minima [see Fig. 1(d)]. The torque is accounted for by tilting the potential [see Figs. 2(a) and 2(b) and Appendix E3]. The continuous rotation is coarse grained into a unicyclic network with three rotational states, whereby the statistics of rotational state changes remain exact. The statistics of exit time from either minimum are depicted in Fig. 7(e).

The probability densities to make a step in the forward $(+)$ and backward (-) direction after time $t$ are given by 

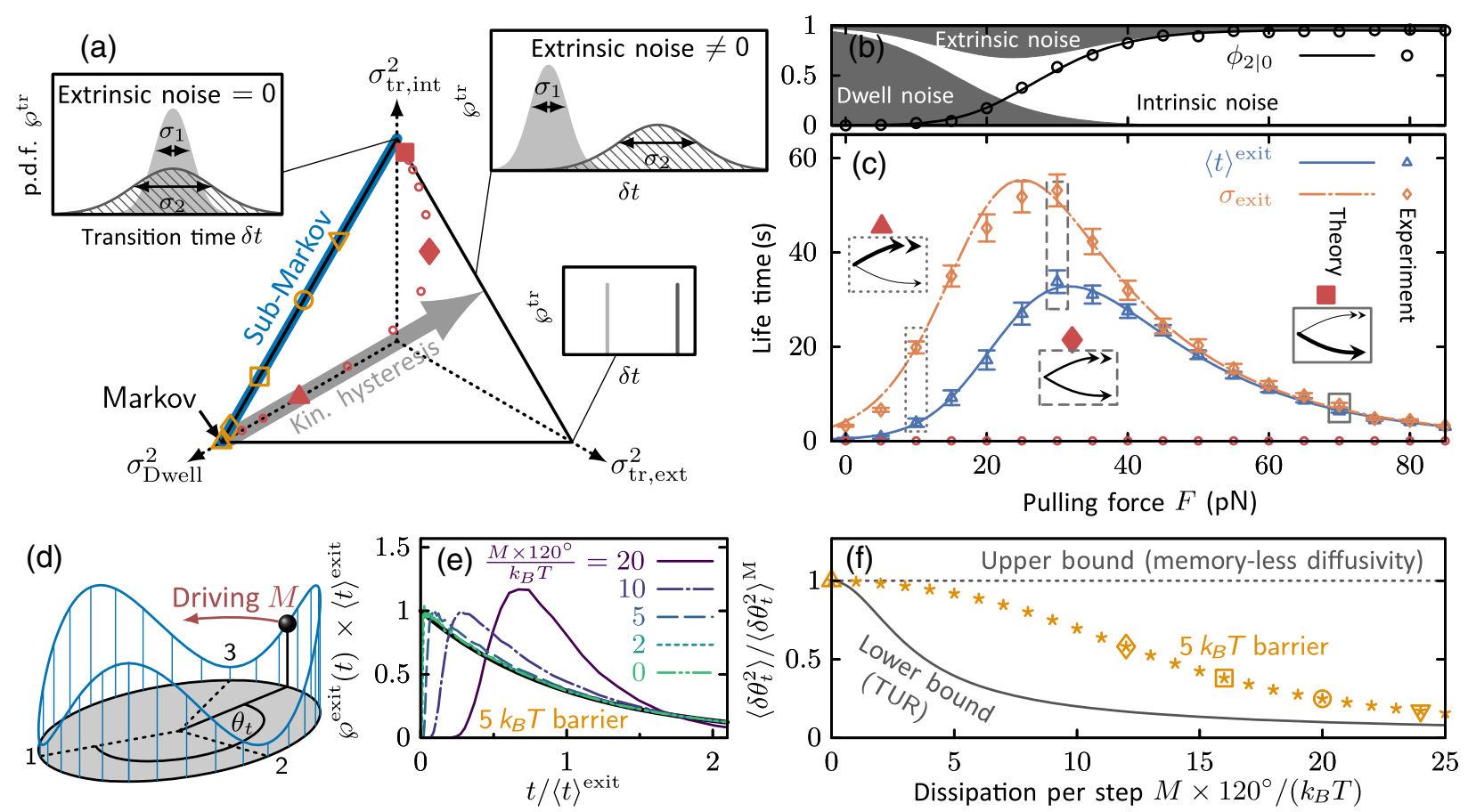

FIG. 7. Manifestations of transition noise and "flavor of memory." (a) The partitioning of noise contributions mapped onto a triangle; the center of the triangle represents the equipartitioning of noise sources $\sigma_{\mathrm{dwell}}^{2}=\sigma_{\mathrm{tr} \text {,int }}^{2}=\sigma_{\mathrm{tr} \text {,ext }}^{2}=\sigma_{\text {exit }}^{2} / 3$. The left corner corresponds to Markov kinetics $\sigma_{\mathrm{tr} \text {,ext }}^{2}=\sigma_{\mathrm{tr} \text {,int }}^{2}=0$, and the left edge (blue line) to "sub-Markov" kinetics $\sigma_{\mathrm{tr} \text {,ext }}^{2}=0$. The top corner corresponds to a vanishing dwell and extrinsic transition noise $\sigma_{\text {dwell }}^{2}=\sigma_{\text {trext }}^{2}=0$, whereas the right corner depicts the limit of vanishing dwell and intrinsic transition noise $\sigma_{\mathrm{dwell}}^{2}=\sigma_{\mathrm{tr}, \text { int }}^{2}=0$. The boxed probability density functions (p.d.f. $\wp^{\mathrm{tr}}$ ) are shown for illustrative purposes. The kinetic hysteresis increases along the gray arrow. The red circles depict the results of the catch-bond example for different pulling forces $F$ shown in panels (b) and (c) [results depicted by larger symbols are additionally illustrated in (c)], and orange symbols show the results for the driven ATPase shown in panel (f). (b),(c) Reconstructed catch-bond experiment. (b) shows $\phi_{2 \mid 0}$, the probability of taking the slow pathway 2 (black line); the shaded areas depict the fraction of dwell $\left(\sigma_{\text {dwell }}^{2} / \sigma_{\text {exit }}^{2}\right)$, extrinsic $\left(\sigma_{\text {tr ext }}^{2} / \sigma_{\text {exit }}^{2}\right)$, and intrinsic $\left(\sigma_{\text {trint }}^{2} / \sigma_{\text {exit }}^{2}\right)$ noise, respectively. (c) depicts the mean $\langle t\rangle^{\text {exit }}$ and standard deviation $\sigma_{\text {exit }}$ of the bond's lifetime. Lines correspond to exact results, and symbols are deduced from 500 rupture experiments. Red circles depict pulling forces considered in panel (a). The density of the lifetime of the bond at $F=20 \mathrm{pN}$ is shown in Fig. 1(d). (d)-(f) Driven molecular motor displaying a vanishing extrinsic transition noise $\sigma_{\text {trext }}=0$. (d) Free-energy landscape as a function of the angle $\theta_{t}$ with a barrier height of $5 k_{B} T$ (blue line; see Appendix E 3 for details) that becomes tilted due the action of the torque $M$ (red arrow); dotted lines denote network states, i.e., free-energy minima. (e) Scaled probability density of exit time from a state (i.e., first-passage time to an angular displacement of $\pm 2 \pi / 3= \pm 120^{\circ}$ ) as a function of the dissipation per step (i.e., torque $M$ multiplied by the rotation step $120^{\circ}$ ) of magnitude $0,2,5,10$, and $20 k_{B} T$. (f) Steady-state meansquared angular deviation $\left\langle\delta \theta_{t}^{2}\right\rangle$ compared with the a Markov-jump approximation $\left\langle\delta \theta_{t}^{2}\right\rangle^{M}$ as a function of the dissipation. The full line depicts the lower bound (E7) derived in Appendix (E4) using the thermodynamic uncertainty relation (TUR) [79,80]; the individual noise contributions for selected points (open symbols) are shown in panel (a). Parameters: (b),(c) see Appendix E2; (d)-(f) see Appendix E3.

$\wp_{ \pm}^{\text {loc }}(t)=\phi_{ \pm} \wp^{\text {exit }}(t)$ yielding a mean-squared angular deviation $[16,37],\left\langle\delta \theta_{t}^{2}\right\rangle \equiv\left\langle\theta_{t}^{2}\right\rangle-\left\langle\theta_{t}\right\rangle^{2} \quad$ [see proof in Appendix E 3, Eq. (E4)]

$$
\frac{\left\langle\delta \theta_{t}^{2}\right\rangle}{\left(120^{\circ}\right)^{2}} \simeq 4 \phi_{+} \phi_{-} \frac{t}{\langle t\rangle^{\text {exit }}}+\left(\phi_{+}-\phi_{-}\right)^{2} \frac{t \sigma_{\text {exit }}^{2}}{\left(\langle t\rangle^{\text {exit }}\right)^{3}}
$$

where $\simeq$ denotes asymptotic equality in the limit $t \rightarrow \infty$. Memoryless Markovian kinetics would predict $\sigma_{\text {exit }}^{M} \equiv$ $\langle t\rangle^{\text {exit }} \geq \sigma_{\text {exit }}$ and thus systematically overestimate fluctuations [dotted line in Fig. 7(f)]. Memory is particularly pronounced in the regime of strong driving, i.e., $\phi_{+} \gg \phi_{-}$ or $\phi_{-} \gg \phi_{+}$. Notably, we find the so-called thermodynamic uncertainty relation $[79,80]$ to bound fluctuations from below [Fig. 7(f), solid line; for a proof, see Appendix E 4]. Our results thereby yield a "sandwich" bound on actual fluctuations in driven cycle-graph (i.e., ring-shaped) networks.

\section{MARGINAL OBSERVATIONS AND HIDDEN CYCLES}

So far, we have discussed a non-Markovian network theory that accounts for transition paths without hidden cycles. We now show how one can generalize our results to 
systems with hidden cycles. Strikingly, we show that an evaluation of the mean waiting time from a partial observation alone can reveal non-Markovian fingerprints of the full network dynamics. Moreover, we discuss future perspectives and show what can be learned from established coarse-graining schemes known as milestoning.

\section{A. Marginal observations reveal fingerprints of non-Markovianity}

Detecting even a single cycle in an experiment can be challenging because it requires us to resolve two parallel transition paths as achieved, e.g., in Ref. [29]. It thus may not always be feasible to monitor more than one existing cycle.

To describe such scenarios, we need to account for possible hidden nodes that cannot be detected as shown in Fig. 8(a). For example, among the five states listed Fig. 3, it may be that we can monitor only three states, say, states $1-3$. In other words, recurrences and state changes to states 4 and 5 are assumed not to be monitored. In this case, we can directly observe the nontrivial cycle $1 \rightarrow 2 \rightarrow 3$, whereas the second elementary cycle $3 \rightarrow 2 \rightarrow 4 \rightarrow 3$ shown in Fig. 3 is not directly visible-it corresponds $3 \rightarrow$ $2 \rightarrow 3$ in the marginal observation [compare dotted and solid line in Fig. 8(a)]. Once a network has hidden cycles with a nonzero affinity (see Ref. [86] and Appendix D 1), we expect Eq. (2) not to hold in conjunction with the possible breakdown of the forward or backward symmetry of the transition-path time within the marginal observation as demonstrated in Ref. [28]. It is worth mentioning that both the dotted and the solid lines depicted in Fig. 8(a) will generally represent a semi-Markov process and that the independence between transition-path time and dwell time as in Eq. (3) is expected to be preserved.

We explain in Appendix C 3 how one can apply the network theory we develop to study marginal observations.
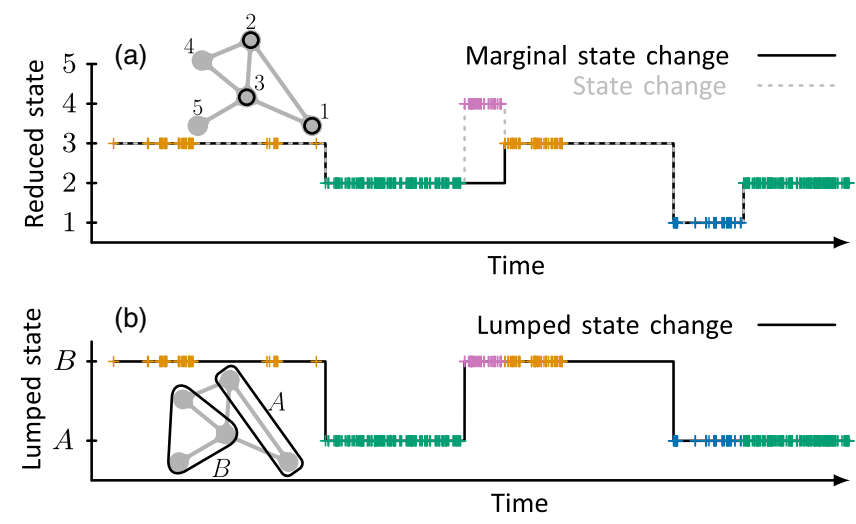

FIG. 8. Marginal observations and lumping of states. (a) Marginal state-change trajectory (black line) if states 4 and 5 are hidden. (b) Lumping of states into $A=\{1,2\}$ and $B=\{3,4,5\}$. The colored crosses correspond to state visits of all five states as in Fig. 3.
More precisely, the waiting-time distribution of a marginal state change to the other states within a subset can be described by all the local waiting-time distributions $\wp_{j \mid i}^{\text {loc }}(t)$ [see Eq. (C10)]. Thus the path decomposition, which generalizes the classical renewal theorem [74] that is fully explained in Appendix $\mathrm{C}$ and used to derive our results, can be used further to generalize our results in order to include marginal observations.

Appendix $\mathrm{C} 4$ shows that the marginal dynamics along $3 \rightarrow 1$ is slower and concurrently also less likely than the marginal dynamics along $3 \rightarrow 2$. Note that both Markovstate kinetics [1,2,4-14] as well as isotropic (decoupled) renewal processes $[37,43,44,94,96]$ would invariably infer transition $3 \rightarrow 1$ to be erroneously faster on average than $3 \rightarrow 2$ (see Appendix C4). This is because the direct transition $3 \rightarrow 1$ takes longer than the paths $3 \rightarrow 2$ that involve a detour through the intermediate state 4 . Markov models, for example, do not allow for this to happen because they assume transitions to occur instantaneously. Therefore, one can detect non-Markovianity of the underlying network dynamics solely on the basis of mean waiting times in the marginal observation. Similarly, symmetric waiting-time distributions in renewal processes render the duration of all transitions from any state equal, such that any path involving a detour is bound to take longer on average. In other words, memory in the marginal observation in Fig. 8 cannot emerge solely from ignoring states 4 and 5. Notably, this has a mean waiting time along $3 \rightarrow 1$ that is different from the mean waiting time along $3 \rightarrow 2$, which was concluded to be a sign of mathematical time-reversal symmetry breaking in Ref. [43] and confused to be a signature of broken detailed balance in Ref. [54]. Since the model satisfies detailed balance, these findings need to be revised for both the marginal observation and the full observation, as soon as transition paths are not instantaneous. We thus expect the kinetic hysteresis, which accounts for transition paths being odd under time reversal as explained in Fig. 6, to extend to marginal observations. Note that if the marginal observation hides away cycles, we expect Eq. (19) to be a lower bound to the entropy production rates, while the rate in Eq. (23) for the marginal observation in Fig. 8(a) becomes nonzero at equilibrium.

A correct interpretation of marginal observations in the presence of memory therefore requires the thermodynamically consistent coarse graining derived in our work. More severe manifestations of memory are found in driven networks [see the catch-bond analysis in Fig. 1(d) or Table V].

\section{B. Lumping nodes into mesostates}

In the following, we discuss the coarse graining that lumps multiple states into a few "mesostates" as shown in Fig. 8(b). In this figure, we lump five states into a pair of clustered mesostates $A=\{1,2\}$ and $B=\{3,4,5\}$. The lumped state changes from a hidden semi-Markov process [99], for which we expect the waiting and dwell time to not 
depend only on the current state; that is, Eq. (3) is expected to be violated.

Lumped dynamics emerging from hidden Markov models have been studied thoroughly from a thermodynamic point of view [100]. However, in contrast to our example in Fig. 8(b), Ref. [100] presumed transition paths to be infinitely fast. The trajectory depicted Fig. 8(b) displays three transitions between clusters $A$ and $B$, which occur along three distinct continuous transition pathways, while the last transition involves the prolonged transition $\delta t$ between states 1 and 3 which is highlighted in Figs. 3(b) and 6.

As soon as states are lumped, the waiting-time density in a lumped state Eq. (3) depends also on the states visited before said state. The dwell-time statistics then depends not only on the current state. For example, the dwell-time statistics in certain lumped networks was found to develop a "transition memory" [101], which depends on the future $j$, current $i$, and the past $k$ state [54]; i.e., the dwell-time symmetry Eq. (3) is violated $\wp_{i}^{\text {dwell }} \rightarrow \wp_{j \mid i k}^{\text {dwell. }}$. In this case, we furthermore expect the independence between dwell time and transition-path time to be violated.

Notably, one of our examples shown in Figs. 7(b) and 7(c) in fact involves lumped states. There we measure the lifetime of a bond that can rupture along two pathways. After rupture, the bond remains in two distinct states that we effectively lump into one ruptured state [see Fig. 1(d)]. Furthermore, any exit-time distribution from a state $i$ represents a passage from $i$ to the lumped set $\mathcal{N}_{i}$ of all states adjacent to $i$.

We demonstrate in Appendix C 4 (see also discussion in the previous subsection) how one can deduce the waitingtime distribution from one state within $B$ into either state in the lumped mesostate $A=\{1,2\}$. Choosing the set of target states to be either of the two mesostates $(\mathcal{A}=A$ or $\mathcal{A}=B$ ), we can deduce the waiting-time statistics to any cluster.

To obtain a physically consistent coarse graining, transition paths should be considered to be odd under time reversal. The kinetic hysteresis is thus also important to properly account for the thermodynamic entropy production rate in the presence of lumping. As soon as the lumping hides cycles, Eq. (19) is expected to bound the entropy production in the lumped system from below $[54,55,100]$. If all lumped states relax quickly and the transitions between all lumped states are fast, the lumped dynamics becomes approximately Markovian [18].

Next we explain how one can conceptually extend the applicability of our results to more general types of underlying microscopic dynamics, i.e., other than diffusion on a graph, via a proxy-the so-called milestoning methodology (see, e.g., Refs. [3,18,41,42,73,101,102]).

\section{Milestoning}

Milestoning is a (numerical) method for deriving discrete state kinetics from an underlying continuous dynamics
$[3,73]$. We now highlight how milestoning allows us to extend our results to systems with microscopic dynamics other than diffusion on a graph. To this end, we identify or replace the nodes (or states) in the network by "milestones" such that each recurrence in Fig. 3 becomes a passage through a milestone [63].

Milestones can represent hypersurfaces [41] or hypervolumes, i.e., cores [18]. In Ref. [41], milestones were introduced as a hypersurface-to-hypersurface hopping, while the underlying dynamics perpendicular to the milestones was assumed to be the slowest degree of freedom that is effectively one dimensional. Our work now additionally introduces cycles and branch points (see Fig. 2), which allow for a nonvanishing rate of steady-state dissipation. Equation (2) shows, for the first time, that a coarse graining based on milestoning robustly preserves the rate of dissipation both arbitrarily far from equilibrium and in the presence of memory. In other words, local detailed balance [6] is allowed to be violated. It will be interesting to further account for the thermodynamics of transition memory [101], which can be caused by fast interhypersurface dynamics or by lumping of hypersurfaces that may lead to what is called a semi-Markov process of second order [54]. In this latter case, a splitting probability of the transition $i \rightarrow j$ that depends also on the state $k$ directly preceding $i$.

A slightly different milestoning approach presented in Ref. [18] is based on cores which are small volumes in phase space. In the scenario when transition-path times become short, milestoning already emerged as a valuable tool to deduce a (memoryless) master equation in a kinetically consistent manner [18] (cf. third paragraph in the Introduction therein). While Eq. (2) may not hold anymore exactly, we expect that it will still provide a useful estimate for entropy production. Our results illustrate that milestoning leads to a robust and thermodynamically consistent coarse graining without requiring the dynamics to be Markovian. We therefore anticipate the milestoning based on cores to become a valuable tool for extending our results also to those types of microscopic dynamics that may not be directly described by a diffusion on a graph. To illustrate the rationale behind this idea, we briefly sketch how a generic discrete-state dynamics obtained by milestoning relates to the results derived from our model of diffusion on a graph and the marginal observation depicted in Fig. 8(a).

In a Markov-jump processes, each recurrence is in fact extended in time and exponentially distributed. To capture this in our model, we must simply adopt finite cores [18] that effectively "smear out" the nodes (i.e., crosses in Fig. 8) to continuous segments. The transition time $(2 \rightarrow 3)$ in the marginal observation becomes the time span over which we would detect the hidden state 4 . That is, the full and marginal state change differs. We may now conceptually replace, to a very good approximation, all hidden parts of the network with a sufficiently dense Markov 
network with the appropriate topology and adopt Ref. [102] which explains how to deduce transition-path-time statistics between any pair of marginally observed states in Markov-jump networks in quite general context. The entropy production of the reduced network dynamics was in turn evaluated in Ref. [53]. We may thus consider Refs. $[18,53,102]$ and the coarse graining outlined in Fig. 3 to provide a generic blueprint for constructing a thermodynamically consistent coarse graining.

\section{CONCLUSION}

Emerging from the mapping of continuous dynamics are three elementary independent sources of fluctuations in state transitions on a network: dwell-time fluctuations and the intrinsic and extrinsic noise arising from random transition-path times. The balance of these noise channels depicted in the noise triangle in Fig. 7(a) yields Markovian, sub-, or super-Markovian fluctuations and thus sets the flavor of memory. A vanishing extrinsic transition noise causes sub-Markovian dynamics as in the driven ATPase [Fig. 7(a), orange symbols]. Markovian dynamics is dominated by dwell noise (left corner). Super-Markovian fluctuations (observed, e.g., in catch-bond dynamics) are dominated by extrinsic transition noise (right corner). The noise triangle allows for a conclusive inference of underlying dominant hidden continuous paths in general networks solely from the observed fluctuations in state transitions. The kinetic hysteresis between forward and time-reversed state trajectories that arises in the presence of transition noise (gray arrow) provides a new understanding of the breaking of time-reversal symmetry in the presence of memory [54,94-96]. The widely adopted principle of local detailed balance is found to be a peculiarity of the Markovian limit, not a general feature of time-reversal symmetry. Our results pave the way toward a deeper understanding of network dynamics far from equilibrium including current fluctuations in active molecular systems $[79,80]$. Even though we deduce the dissipation in the longtime limit here, the generalization of the local detailed balance relation in Eq. (2) holds at any time and includes networks with infinitely many states as well as transient dynamics. Our work thus enables investigations of transient network dynamics.

\section{ACKNOWLEDGMENTS}

The financial support from the German Research Foundation through the Emmy Noether Program No. GO $2762 / 1-1$ to A. G. is gratefully acknowledged.

\section{APPENDIX A: FROM DIFFUSION ON A GRAPH TO STATE CHANGES ON A NETWORK: RIGOROUS COARSE GRAINING}

In this Appendix, we first describe the stochastic dynamics of individual trajectories and its numerical implementation. The translation of the Langevin equation for the time evolution of individual trajectories into a Fokker-Planck equation for the time evolution of probability densities on a graph is explained in the last subsection.

\section{Stochastic differential equation on a graph}

We parametrize $\boldsymbol{x}_{t}$ (the microstate at time $t$ ) in such a way that the reduced state $i_{t}$ represents the last visited network state $i_{t} \in \Omega=\{1, \ldots, N\}$. The microstate is assumed to be fully characterized by $\boldsymbol{x}_{t}=\left(x_{t}, j_{t}, i_{t}\right)$, where $x_{t}$ denotes the distance from the last visited network state $i_{t}$ along the link to network state $j_{t}$ as shown in Fig. 4(a), where $i_{t}=i$ and $j_{t}=j$. The variable $\boldsymbol{x}_{t}=\left(x_{t}, j_{t}, i_{t}\right)$ fully determines the microstate configuration on the graph at time $t$. Denoting the distance between two nodes $i$ and $j$ by $l_{i \mid j}=l_{j \mid i}$, the distance function $x_{t}$ must lie within the interval $0 \leq x_{t} \leq l_{j_{t} \mid i_{t}}$. The "instantaneously" targeted state $j_{t}$ and the last visited state $i_{t}$ do not change until $x_{t}$ reaches either the "inner boundary" $x_{t}=0$ or the "outer boundary" $x_{t}=l_{j_{t} \mid i_{t}}$. After this, the variables change according to the rule described in Table II. In Fig. 3(a), each cross corresponds to the visit of a state, where each revisit of the state (recurrence) with the same color corresponds to all incidents $x_{t}=0$ in which the "inner boundary is hit." Conversely, each state change corresponds to hitting the outer boundary $x_{t}=l_{j_{t} \mid i_{t}}$ [see thick black crosses in Fig. 3(a)], after which the latest visited state becomes $i_{t+0}=j_{t}$, and the instantaneously targeted state is chosen, without loss of generality, with equal probability among the neighbors $j_{t+0} \in \mathcal{N}_{j_{t}}$. The microstate description

TABLE II. Detailed description of the update rules of the microstate during time step " $t \rightarrow t+d t$." Each variable $\beta_{t}$ is randomly chosen among the states adjacent to $k, \mathcal{N}_{k}$, with a uniform probability $1 /\left|\mathcal{N}_{k}\right|$, where $\left|\mathcal{N}_{k}\right|$ denotes the number of elements in the set $\mathcal{N}_{k}$ and $k=i_{t}, j_{t}$. Note that the rules listed in the table do not change the microstate (see Fig. 4).

\begin{tabular}{|c|c|c|c|}
\hline \multirow[b]{2}{*}{ Variable } & \multirow[b]{2}{*}{ Meaning } & \multicolumn{2}{|c|}{ Update conditions at the border } \\
\hline & & Outer boundary $\left(x_{t} \geq l_{i_{t} \mid j_{t}}\right)$ & Inner boundary $\left(x_{t} \leq 0\right)$ \\
\hline$\overline{i_{t}}$ & Latest visited state & $i_{t+d t}=j_{t}$ & $i_{t+d t}=i_{t}$ \\
\hline$x_{t}$ & Current distance from latest visited state & $x_{t+d t}=\left(l_{i_{t} \mid j_{t}}-x_{t}\right)$ & $x_{t+d t}=-x_{t}$ \\
\hline$j_{t}$ & Currently targeted new network state & $\begin{array}{c}\text { Generate randomly } \beta_{t} \in \mathcal{N}_{j_{t}} \\
\text { and set } j_{t+d t}=\beta_{t}\end{array}$ & $\begin{array}{c}\text { Generate randomly } \beta_{t} \in \mathcal{N}_{i_{t}} \\
\text { and set } j_{t+d t}=\beta_{t}\end{array}$ \\
\hline
\end{tabular}


deliberately contains a redundancy since microstates $\boldsymbol{x}_{t}=$ $\left(x_{t}, j_{t}, i_{t}\right)$ and $\tilde{\boldsymbol{x}}_{t}=\left(l_{j_{t} \mid i_{t}}-x_{t}, i_{t}, j_{t}\right)$ correspond to the same microstate configuration, even though the last component of $\tilde{\boldsymbol{x}}_{t}$ does not represent the last visited state (see Fig. 4).

The microstate $\boldsymbol{x}_{t}=\left(x_{t}, j_{t}, i_{t}\right)$ evolves such that both the last visited state $i_{t}$ and the instantaneously targeted state $j_{t}$ remain unchanged during each interval, in which the distance $x_{t}$ lies within the interval $0<x_{t}<l_{j_{t} \mid i_{t}}$, which corresponds to $x_{t} \neq 0$ and $x_{t} \neq l_{j_{t} \mid i_{t}}$. During the time when both $j_{t}$ and $i_{t}$ are constant, the distance $x_{t}$ between two connected nodes evolves according to the anti-Itô Langevin equation (4), which can also translate into the following equivalent Itô-Langevin equation:

$\dot{x}_{t}=\beta D_{j_{t} \mid i_{t}}\left(x_{t}\right) F_{j_{t} \mid i_{t}}\left(x_{t}\right)+D_{j_{t} \mid i_{t}}^{\prime}\left(x_{t}\right)+\sqrt{2 D_{j_{t} \mid i_{t}}\left(x_{t}\right)} \xi_{t}$,

where $D_{j \mid i}^{\prime}(x)=\partial_{x} D_{j \mid i}(x), i=i_{t}$, and $j=j_{t}$. Note that the anti-Itô differential equation is also referred to as the Hänggi-Klimontovich [76,77] equation (see also more recently Refs. [103,104]), while Ref. [77] derives the Stratonovich variant of Eq. (A1) called the "kinetic form." Equations (4) and (A1) follow from the assumption that the inverse friction coefficient (mobility) satisfies the Einstein relation $\mu_{j \mid i}(x)=\beta D_{j \mid i}(x)$ readily inserted in the first term of Eq. (A1).

Equations (4) and (A1) describe the time evolution of the first component of the microstate $\boldsymbol{x}_{t}=\left(x_{t}, j_{t}, i_{t}\right)$. Numerical schemes to propagate Eqs. (4) and (A1) are presented below, where Appendix A 2 shows a naive simple Euler method, and Appendix A 3 the celebrated Milstein scheme [105]. For systems with multiplicative noise (i.e., with a microstate-dependent noise amplitude), we generate trajectories according to the Milstein scheme described in Appendix A 3. For systems with additive noise [constant noise amplitude, that is, $D_{j \mid i}(x)=$ const], we use the scheme shown in Appendix A 4 (adopted from Ref. [78]). Functionals of trajectories, such as the dwelland transition-path-time periods, are evaluated irrespective of the chosen numerical integration scheme of the Langevin equation as we explain in Appendix A 5.

\section{Naive anti-Itô Euler scheme (strong order 0.5)}

The simplest way to numerically integrate the anti-Itô Langevin equation (4) from $t$ to time $t+\Delta t$ is the following anti-Itô Euler scheme:

$$
\begin{aligned}
\tilde{x}_{t} & =x_{t}+\beta D_{j_{t} \mid i_{t}}\left(x_{t}\right) F_{j_{t} \mid i_{t}}\left(x_{t}\right) \Delta t+\sigma_{j_{t} \mid i_{t}}\left(x_{t}\right) \hat{Z}_{t}, \\
x_{t+\Delta t} & =\tilde{x}_{t}+\left[\sigma_{j_{t} \mid i_{t}}\left(\tilde{x}_{t}\right)-\sigma_{j_{t} \mid i_{t}}\left(x_{t}\right)\right] \hat{Z}_{t},
\end{aligned}
$$

where $\sigma_{j \mid i}(x) \equiv \sqrt{2 D_{j \mid i}(x) \Delta t}$, and $\hat{Z}_{t}$ is a standard normally distributed random number, i.e., $\hat{Z}_{t} \sim \mathcal{N}(0,1)$.
The first line of Eq. (A2) estimates the updated position with the value $\tilde{x}_{t}$, after which the second line effectively "replaces" the last term of the first line by $\sigma_{j_{t} \mid i_{t}}\left(\tilde{x}_{t}\right) \hat{Z}_{t}$. Equation (A2) becomes the well-known Euler-Maruyama method if $D_{i \mid j}(x)$ is constant, since the second line then simplifies to $x_{t+\Delta t}=\tilde{x}_{t}$. Once the position exceeds the outer boundary $x_{t+\Delta t}, \tilde{x}_{t}>l_{j_{t} \mid i_{t}}$ or the inner boundary $x_{t+\Delta t}, \tilde{x}_{t}<0, j_{t}$ and $i_{t}$ are updated according to Table II. We note the pathwise error of the Euler scheme (A2) (i.e., the strong error) [105] is expected to scale as $\propto \Delta t^{0.5}$; i.e., the scheme is of the strong order 0.5 .

\section{Milstein scheme (strong order 1.0)}

Since to our knowledge higher-order stochastic RungeKutta schemes can be found only in the literature for Itô or Stratonovich integrals [105], we now use the Itô representation of the equation of motion (A1). In the case of multiplicative noise $D_{j \mid i}^{\prime}(x) \neq 0$, the Euler scheme from Appendix A 2 can be improved according to the Milstein scheme [105], which is of strong order 1.0 (i.e., pathwise error scales as $\propto \Delta t^{1.0}$ ). This scheme propagates the system from time $t$ to time $t+\Delta t$ according to

$$
\begin{aligned}
x_{t+\Delta t}= & x_{t}+\left[\beta D_{j_{t} \mid i_{t}}\left(x_{t}\right) F_{j_{t} \mid i_{t}}\left(x_{t}\right)+D_{j_{t} \mid i_{t}}^{\prime}\left(x_{t}\right)\right. \\
& +\sqrt{2 D_{j_{t} \mid i_{t}}\left(x_{t}\right) \Delta t} Z_{t}+\frac{D_{j_{t} \mid i_{t}}^{\prime}\left(x_{t}\right)}{2}\left[Z_{t}^{2}-1\right] \Delta t \\
= & x_{t}+\beta D_{j_{t} \mid i_{t}}\left(x_{t}\right) F_{j_{t} \mid i_{t}}\left(x_{t}\right) \Delta t \\
& +\sqrt{2 D_{j_{t} \mid i_{t}}\left(x_{t}\right) \Delta t} Z_{t}+\frac{D_{j_{t} \mid i_{t}}^{\prime}\left(x_{t}\right)}{2}\left[Z_{t}^{2}+1\right] \Delta t,
\end{aligned}
$$

where $Z_{t} \sim \mathcal{N}(0,1)$. The last term in the second line of Eq. (A3) reduces the pathwise error from $\epsilon \propto \Delta t^{0.5}$ to $\epsilon \propto \Delta t^{1.0}$. In the last step in Eq. (A3), we solely combine the terms containing the derivative of the diffusion coefficient. Once the position exceeds the outer boundary $x_{t+\Delta t}>l_{j_{t} \mid i_{t}}$ or the inner boundary $x_{t+\Delta t}<0, j_{t}$ and $i_{t}$ are updated according to Table II.

\section{Stochastic Runge-Kutta with additive noise (strong order 1.5)}

For the simulation of stochastic trajectories with a constant diffusion coefficient $D_{j \mid i}(x)=$ const $=D$ (i.e., additive noise), we use an explicit stochastic RungeKutta scheme of strong order 1.5 from Ref. [105] (see also Ref. [78]), which involves the following steps assuming a time increment $\Delta t$. In order to update from $x_{t}$ to $x_{t+\Delta t}$, we first generate two independent standard normally distributed random numbers $\hat{Z}_{t} \sim \mathcal{N}(0,1)$ and $\zeta_{t} \sim \mathcal{N}(0,1)$, calculate $\hat{R}_{t}=\hat{Z}_{t} / 2+\zeta_{t} \times \sqrt{3} / 6$, and then update the position according to [78] 


$$
\begin{aligned}
q_{t} & =x_{t}+\frac{1}{2} \beta D F_{j_{t} \mid i_{t}}\left(x_{t}\right) \Delta t, \\
q_{t}^{*} & =q_{t}+\frac{3}{2} \sigma \hat{R}_{t}, \\
x_{t+\Delta t} & =x_{t}+\sigma \hat{Z}_{t}+\left[\frac{\beta D F_{j_{t} \mid i_{t}}\left(q_{t}\right)+2 \beta D F_{j_{t} \mid i_{t}}\left(q_{t}^{*}\right)}{3}\right] \Delta t,
\end{aligned}
$$

where $\sigma=\sqrt{2 D \Delta t}$. We emphasize that this stochastic Runge-Kutta scheme is of strong order 1.5 and assumes the diffusion coefficient to be constant. Moreover, this scheme requires two random numbers instead of one in each iteration step. A quite comprehensive collection of further higher-order stochastic integration schemes can be found in Ref. [105], which in contrast to Eq. (A4) require generating non-Gaussian random numbers.

\section{Evaluation of dwell- and transition-path-time functionals}

The waiting time in one reduced network state spans the time period between the first entrance into a network state on a graph until the first entrance to another state (see Fig. 3), i.e., the time between two state changes. The dwell and transition-path time dissect the residence-time interval into two separate intervals, in which the last recurrence (revisit) of the same state before changing to another state terminates the dwell time $\tau$ and initiates the transition-pathtime period $\delta t$ that in turn spans the remaining time until the state changes. To numerically evaluate the dwell- and transition-path-time functionals defined in Eqs. (6) and (8), we perform the following computational steps.

Whenever the position $x_{t}$ exceeds the outer boundary $x_{t} \geq l_{j_{t} \mid i_{t}}$ (i.e., the state changes) or the inner boundary $x_{t} \leq$ 0 (i.e., to a recurrence), which both represent a "state visit," the variables $j_{t}, i_{t}$ are updated according to Table II. Any update of $j_{t}, i_{t}$ according to Table II is accompanied by a change of dwell time $\tau$ and transition-path time $\delta t$ according to Table III. Thereby, $T_{\text {rec }}$ denotes the last recurrence of a network state and $T_{\text {last }}$ the time of the last state change. Each transition event is stored in a list for all transitions $\gamma$ (see second step in Table III).

\section{Fokker-Planck equation on local starlike graph}

The preceding subsection deals with single trajectories. One can cast the Langevin equation (A1) into a partial differential equation for the probability density functionthe so-called Fokker-Planck equation-as follows [83]. We pick without loss of generality a state of interest $i$ and focus on a local starlike graph spanned by the $i$ th state. For a pair of neighboring states $j, k \in \mathcal{N}_{i}$ at distances $x$ and $y$ within $0 \leq x \leq l_{j \mid i}$ and $0 \leq y \leq l_{k \mid i}$, the probability density to find the system in the state $(x, j, i)$ after time $t$ starting initially from $(y, k, i)$ denoted by $P_{i}(x, j, t \mid y, k)$ satisfies the Fokker-Planck equation

$$
\begin{aligned}
& \partial_{t} P_{i}(x, j, t \mid y, k) \\
& =-\partial_{x} J_{i}(x, j, t \mid y, k) \\
& =\partial_{x} e^{-\beta U_{j i}(x)} D_{j \mid i}(x) \partial_{x} e^{\beta U_{j i}(x)} P_{i}(x, j, t \mid y, k) \\
& \equiv \hat{\mathcal{L}}_{j \mid i}^{F}(x) P_{i}(x, j, t \mid y, k)
\end{aligned}
$$

where $U_{j \mid i}(x)=-\int_{0}^{x} F_{j \mid i}\left(x^{\prime}\right) d x^{\prime}$, and without any loss of generality, we assume the diffusion constant to be continuous $D_{j \mid i}(0)=D_{k \mid i}(0)$ for all $j, k \in \mathcal{N}_{i}$. Note that $J_{i}(x, j, t \mid y, k) \equiv D_{j \mid i}(x)\left[\beta F_{j \mid i}(x)-\partial_{x}\right] P_{i}(x, j, t \mid y, k)$ denotes the probability flux away from $i$, and $\hat{\mathcal{L}}_{j \mid i}^{F}(x)$ denotes the (forward) Fokker-Planck operator. The initial probability density is set to $P_{i}(x, j, t \mid y, k)=\delta(x-y) \delta_{j k}$, where $\delta(x-y)$ is the delta function and $\delta_{j k}$ the Kronecker delta. The inner boundary conditions read

$$
\begin{aligned}
P_{i}(0, j, t \mid y, k) & =P_{i}\left(0, j^{\prime}, t \mid y, k\right), \quad \forall j^{\prime} \in \mathcal{N}_{i}, \\
\sum_{j} J_{i}(0, j, t \mid y, k) & =0,
\end{aligned}
$$

which reflect that trajectories are continuous and fluxes are conserved according to Kirchhoff's law. Note that the generalization to both diverging force kicks and discontinuous diffusion landscapes is explicitly discussed in Appendix D3. Hence, we may derive all results based on Eq. (A6) in order to render the derivations less tedious.

TABLE III. Update of dwell- and transition-path-time functionals. The initial position is assumed to be $x_{0}=0$, and time of the last recurrence is initially set to $T_{\text {rec }}=0$ along with the time of the last state change to $T_{\text {last }}=0$. Each passage across an outer boundary results in a transition-path time $\delta t$ and dwell time $\tau$ that correspond to one transition event along a single transition $\gamma$.

\begin{tabular}{lccc}
\hline \hline & & \multicolumn{2}{c}{ Update conditions at the boundary } \\
\cline { 3 - 3 } Step & Functional & Outer boundary $\left(x_{t} \geq l_{j_{t} \mid i_{t}}\right)$ & Inner boundary $\left(x_{t} \leq 0\right)$ \\
\hline 1st & $\ldots$ & & Set $T_{\text {rec }}=t$ \\
2nd & Splitting transition & Store one transition $\gamma=\left(i_{t} \rightarrow j_{t}\right)$ & \\
3rd & Dwell time $\tau$ & Store $\tau=T_{\text {rec }}-T_{\text {last }}$ in transition $\gamma$ \\
4th & Transition-path time $\delta t$ & Store $\delta t=t-T_{\text {rec }}$ in transition $\gamma$ \\
5th & $\cdots$ & Set $T_{\text {last }}=T_{\text {rec }}=t$ \\
\hline \hline
\end{tabular}


There are two distinct boundary conditions at the outer end of the $j$ th leg adjacent to node $i$ (i.e., $j \in \mathcal{N}_{i}$ ), which correspond to

$$
\begin{array}{ll}
P_{i}\left(l_{j \mid i}, j, t \mid y, k\right)=0 \quad\left[\begin{array}{l}
\text { if the } j \text { th outer } \\
\text { boundary is absorbing, }
\end{array}\right. \\
J_{i}\left(l_{j \mid i}, j, t \mid y, k\right)=0 \quad\left[\begin{array}{l}
\text { if the } j \text { th outer } \\
\text { boundary is reflecting. }
\end{array}\right.
\end{array}
$$

For all absorbing ends, $P_{i}(x, j, t \mid y, k) d x$ is the probability that a trajectory starting from distance $y$ from state $i$ in the direction toward state $k$ will be at time $t$ within the interval $x$ and $x+d x$ having never reached any of the neighboring states $\neq i$. In this case, the survival probability $S_{i}(t \mid y, k)=$ $\sum_{j} \int_{0}^{l_{j i}} P_{i}(x, j, t \mid y, k) d x$ decays monotonically in time from $S_{i}(0 \mid y, k)=1$ to $S_{i}(\infty \mid y, k)=0$. More precisely, if $P_{i}\left(l_{j \mid i}, j, t \mid y, k\right)=0, \forall j \in \mathcal{N}_{i}$, we obtain $[83,106]$

$$
\begin{aligned}
\wp_{j \mid i}^{\text {loc }}(t) & =J_{i}\left(l_{j \mid i}, j, t \mid 0, k\right) \\
& =-\left.D_{j \mid i}\left(l_{j \mid i}\right) \partial_{x} P_{i}(x, j, t \mid y, k)\right|_{x=l_{j \mid i}},
\end{aligned}
$$

where $\wp_{j \mid i}^{\text {loc }}$ is the local state-to-state kinetics with $-\partial_{t} S_{i}(t \mid 0, k)=\sum_{j} \wp_{j \mid i}^{\text {loc }}(t)=\wp_{i}^{\text {exit }}(t) \quad$ for $\quad$ all $k$; the Laplace transform of $\wp_{j \mid i}^{\text {loc }}$ is given in Eq. (C5).

\section{APPENDIX B: PROOF OF SYMMETRY AND INDEPENDENCE OF DWELL AND TRANSITION-PATH TIME USING GREEN'S FUNCTION THEORY}

In the following, we prove that diffusive dynamics on a graph (A5) renders dwell and transition-path times conditionally independent functionals. We first show that the aforementioned conditional independence follows directly from the definition of the coarse graining (last visited state) based on the gedanken experiment from Fig. 3. Using Green's function theory, we then prove the following two symmetries entering Eq. (3): (i) The dwell-time statistics solely depend on the initial state, and (ii) the transition-path time obeys a forward or backward symmetry.

\section{Proof of conditional independence between transition-path and dwell time}

The independence of transition-path and dwell time follows immediately from the coarse graining of the full trajectory once we realize that it effects an "erasure of memory."

The microstate $\boldsymbol{x}_{t}=\left(x_{t}, j_{t}, i_{t}\right)$ is characterized by the last visited state $i_{t}$, and the distance $x_{t}$ from the last visited state in the direction to the instantaneously targeted state $j_{t}$. Each recurrence in Fig. 3 highlighted by colored crosses represents a state visit $x_{t}=0$, which in turn fully determines the microstate via $\boldsymbol{x}_{t}=\left(0, j_{t}, i_{t}\right) \hat{=}\left(0, k, i_{t}\right) \hat{=}\left(l_{j_{t} \mid i_{t}}, i_{t}, j_{t}\right)$, where the symbol " $\hat{=}$ " refers to parameters corresponding to the same instantaneous microstate (see Fig. 4). Since the microstate $\boldsymbol{x}_{t}$ is assumed to follow Markovian kinetics, we find that the future state visit depends only on the last state visit not on the state visits before, which triggers a renewal of the dynamics. Since a transition spans the time after the last revisit of a state (recurrence) and the dwell time spans the time before the last revisit of a state [see Fig. 3(b)], the said revisit of a state causes their statistical independence. This completes the proof of independence between transition-path and dwell time. In the following, we derive the symmetries of transition-path and dwell times using the underlying Fokker-Planck equation on a graph.

\section{Laplace transform of the Fokker-Planck equation on a graph}

Let us first write the Fokker-Planck equation in terms of the current operator $\hat{\mathcal{J}}_{j \mid i}^{\mathrm{F}}(x)=-D_{j \mid i}(x) e^{-\beta U_{j i}(x)} \partial_{x} e^{\beta U_{j \mid i}(x)}$, which allows us to rewrite Eq. (A5) in the form

$$
\partial_{t} P_{i}(x, j, t \mid y, k)=-\partial_{x} \hat{\mathcal{J}}_{j \mid i}^{F}(x) P_{i}(x, j, t \mid y, k) .
$$

The Laplace transform $\tilde{P}_{i}(x, j, s \mid y, k)=\int_{0}^{\infty} e^{-s t} \times$ $P_{i}(x, j, t \mid y, k) d t$ transforms the Fokker-Planck equation (B1) into

$$
\left[s+\partial_{x} \hat{\mathcal{J}}_{j \mid i}^{F}(x)\right] \tilde{P}_{i}(x, j, s \mid y, k)=\delta(x-y) \delta_{j k} .
$$

From Eq. (B2) follows the continuity of probability $\left.\tilde{P}_{i}(x, j, s \mid y, j)\right|_{x=y+0}=\left.\tilde{P}_{i}(x, j, s \mid y, j)\right|_{x=y-0}$ and jump discontinuity at $x=y$ of the current $\hat{\mathcal{J}}_{j \mid i}^{F}(x)$ $\left.\tilde{P}_{i}(x, j, s \mid y, j)\right|_{x=y+0}-\left.\hat{\mathcal{J}}_{j \mid i}^{F}(x) \tilde{P}_{i}(x, j, s \mid y, j)\right|_{x=y-0}=1$. Let us now express the solutions $\tilde{P}_{i}$ in terms of the homogeneous solutions $\psi_{j \mid i}^{\text {in }}(x), \psi_{j \mid i}^{\text {out }}(x)$ satisfying

$\left[s+\partial_{x} \hat{\mathcal{J}}_{j \mid i}^{\mathrm{F}}(x)\right] \psi_{j \mid i}^{\mathrm{in}}(x)=0, \quad\left[s+\partial_{x} \hat{\mathcal{J}}_{j \mid i}^{\mathrm{F}}(x)\right] \psi_{j \mid i}^{\mathrm{out}}(x)=0$,

with $\psi_{j \mid i}^{\text {in }}(0)=0$ and $\psi_{j \mid i}^{\text {out }}\left(l_{j \mid i}\right)=0$. Introducing further the current functions $J_{j \mid i}^{\mathrm{in}}(x) \equiv \hat{\mathcal{J}}_{j \mid i}^{F}(x) \psi_{j \mid i}^{\mathrm{in}}(x)$ and $J_{j \mid i}^{\text {out }}(x) \equiv$ $\hat{\mathcal{J}}_{j \mid i}^{F}(x) \psi_{j \mid i}^{\text {in }}(x)$, one can easily check that the limit $y \rightarrow 0$ yields the solution

$$
\tilde{P}_{i}(x, j \mid 0, k)=\frac{\psi_{j \mid i}^{\text {out }}(x, s) / \psi_{j \mid i}^{\text {out }}(0, s)}{\sum_{n \in \mathcal{N}_{i}} J_{n \mid i}^{\text {out }}(0, s) / \psi_{n \mid i}^{\text {out }}(0, s)},
$$

which is equal for all $k \in \mathcal{N}_{i}$ and continuous in $x=0$. Conversely, the currents are discontinuous at $x=0$ according to Eq. (B2). Equation (B4) also solves $\tilde{P}_{i}\left(l_{j \mid i}, j \mid 0, k\right)=$ 0 for all $j, k \in \mathcal{N}_{i}$. The Laplace transform of the firstpassage-time density $\tilde{\wp}_{j \mid i}^{\text {loc }}(s)$ is obtained from the outward current $[83,106]$ at position $x=l_{j \mid i}$, finally yielding 


$$
\begin{aligned}
\tilde{\wp}_{j \mid i}^{\text {loc }}(s) & \left.\equiv \hat{\mathcal{J}}_{j \mid i}^{\mathrm{F}}(x) \tilde{P}_{i}(x, j \mid 0, k)\right|_{x=l_{j \mid i}} \\
& =\frac{J_{j \mid i}^{\text {out }}\left(l_{j \mid i}, s\right) / \psi_{j \mid i}^{\text {out }}(0, s)}{\sum_{k \in \mathcal{N}_{i}} J_{k \mid i}^{\text {out }}(0, s) / \psi_{k \mid i}^{\text {out }}(0, s)} .
\end{aligned}
$$

The zeroth-order moment of Eq. (B5)—-the splitting probability - is in turn simply given by

$$
\phi_{j \mid i}^{\mathrm{loc}} \equiv \tilde{\wp}_{j \mid i}^{\mathrm{loc}}(0)=\frac{J_{j \mid i}^{\text {out }}\left(l_{j \mid i}, 0\right) / \psi_{j \mid i}^{\text {out }}(0,0)}{\sum_{k \in \mathcal{N}_{i}} J_{k \mid i}^{\text {out }}(0,0) / \psi_{k \mid i}^{\text {out }}(0,0)} .
$$

In the following, we decompose Eq. (B5) exactly into the splitting probability, transition-path-time, and dwell-time statistics.

\section{Transition-path-time statistics from Green's function along a single leg with absorbing boundary conditions}

Before taking the limit in Eq. (7), we Laplace transform the Green's function $\tilde{G}_{j \mid i}^{\mathrm{tr}}(x, s \mid y)=\int_{0}^{\infty} e^{-s t} G_{j \mid i}^{\mathrm{tr}}(x, t \mid y) d t$ which, using the solutions Eq. (B3), can be written in the form [107-109]

$$
\tilde{G}_{j \mid i}^{\mathrm{tr}}(x, s \mid y)= \begin{cases}\frac{\psi_{j \mid i}^{\text {in }}(y, s) \psi_{j \mid i}^{\text {out }}(x, s)}{w_{j \mid i}(y, s)} & \text { if } x \geq y, \\ \frac{\psi_{j \mid i}^{\text {in }}(x, s) \psi_{j \mid i}^{\text {out }}(y, s)}{w_{j \mid i}(y, s)} & \text { if } x \leq y,\end{cases}
$$

where we define the Wronskian satisfying [107,109]

$$
\begin{array}{r}
w_{j \mid i}(y, s)=\psi_{j \mid i}^{\text {in }}(y, s) J_{j \mid i}^{\text {out }}(y, s)-J_{j \mid i}^{\text {in }}(y, s) \psi_{j \mid i}^{\text {out }}(y, s) \\
=w_{j \mid i}(x, s) e^{\beta U_{j \mid i}(x)-\beta U_{j \mid i}(y)} .
\end{array}
$$

At the boundaries, the Wronskian becomes $w_{j \mid i}\left(l_{j \mid i}, s\right)=$ $\psi_{j \mid i}^{\text {in }}\left(l_{j \mid i}, s\right) J_{j \mid i}^{\text {out }}\left(l_{j \mid i}, s\right)$ and $w_{j \mid i}(0, s)=-J_{j \mid i}^{\text {in }}(0, s) \psi_{j \mid i}^{\text {out }}(0, s)$ due to $\psi_{j \mid i}^{\text {out }}\left(l_{j \mid i}, s\right)=\psi_{j \mid i}^{\text {in }}(0, s)=0$. Using

$\tilde{J}_{j \mid i}^{\mathrm{tr}}(x, s \mid y)=\hat{\mathcal{J}}_{j \mid i}^{\mathrm{F}} \tilde{G}_{j \mid i}^{\mathrm{tr}}(x, s \mid y)=\frac{\psi_{j \mid i}^{\mathrm{in}}(y, s) J_{j \mid i}^{\mathrm{out}}(x, s)}{w_{j \mid i}(y, s)}$

for $x>y$, the Laplace image of the probability density of the transition-path time becomes

$$
\begin{aligned}
& \tilde{\wp}_{j \mid i}^{\operatorname{tr}}(s)=\lim _{y \rightarrow 0} \frac{\tilde{J}_{j \mid i}^{\mathrm{tr}}\left(l_{j \mid i}, s \mid y\right)}{\tilde{J}_{j \mid i}^{\mathrm{tr}}\left(l_{j \mid i}, 0 \mid y\right)}=\lim _{y \rightarrow 0} \frac{\psi_{j \mid i}^{\mathrm{in}}(y, s)}{\psi_{j \mid i}^{\mathrm{in}}(y, 0)} \times \frac{w_{j \mid i}(y, 0)}{w_{j \mid i}(y, s)} \times \frac{J_{j \mid i}^{\text {out }}\left(l_{j \mid i}, s\right)}{J_{j \mid i}^{\text {out }}\left(l_{j \mid i}, 0\right)}
\end{aligned}
$$

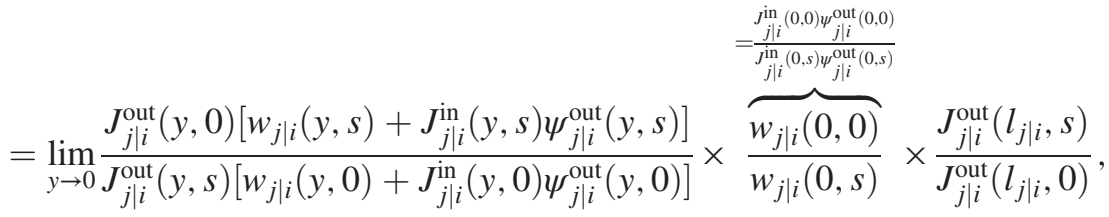

where we perform the following algebraic steps. From the first to the second line of Eq. (B10) we rewrite the first fraction (which is formally undetermined " $0 / 0$ " in the limit $y \rightarrow 0$ ) first by using Eq. (B8) with $x=0$, inserting the resulting $\psi_{j \mid i}^{\text {in }}(y, s)$, and using $\lim _{y \rightarrow 0} w_{j \mid i}(y, s)=w_{j \mid i}(0, s)$. Since $J_{j \mid i}^{\text {out }}(y, s)$ does not have a singularity in the limit $\lim _{y \rightarrow 0} J_{j \mid i}^{\text {out }}(y, s)$, the singularity $0 / 0$ is solely encoded in the bracketed term "[...] $\rightarrow 0$," and cancels in the limit $y \rightarrow 0$ in both the numerator and denominator. Employing l'Hôpital's rule (on the bracketed terms "[...]"), we now determine their first derivative with respect to $y$ at $y=0$,

$$
\begin{aligned}
& D_{j \mid i}(0) \frac{\partial}{\partial y}\left[w_{j \mid i}(y, s)+J_{j \mid i}^{\text {in }}(y, s) \psi_{j \mid i}^{\text {out }}(y, s)\right]_{y=0} \\
& =\beta D_{j \mid i}(0)[-\overbrace{w_{j \mid i}(0, s)}^{=-J_{j \mid i}^{\text {in }}(0, s) \psi_{j \mid i}^{\text {out }}(0, s)} \beta U_{j \mid i}^{\prime}(0)-\overbrace{s \psi_{j \mid i}^{\text {in }}(0, s)}^{=0} \psi_{j \mid i}^{\text {out }}(0, s) \\
& \left.+J_{j \mid i}^{\text {in }}(0, s) \frac{\partial}{\partial y} \psi_{j \mid i}^{\text {out }}(y, s)\right]_{y=0}=-J_{j \mid i}^{\text {in }}(0, s) J_{j \mid i}^{\text {out }}(0, s),
\end{aligned}
$$

where $U_{j \mid i}{ }^{\prime}(y)=\partial_{y} U_{j \mid i}(y)$, and we deduce $\partial_{y} w_{j \mid i}(y, s)=$ $-U_{j \mid i}{ }^{\prime}(y) w_{j \mid i}(y, s)$ from the left side of Eq. (B8). Furthermore, we use $\partial_{y} J_{j \mid i}^{\text {in }}(y, s)=-s \psi_{j \mid i}^{\text {in }}(y, s)$ following from Eq. (B3), and finally employ $J_{j \mid i}^{\text {out }}(y, s)=-D_{j \mid i}(y) \times$ $\beta U_{j \mid i}{ }^{\prime}(y) \psi_{j \mid i}^{\text {out }}(y, s)-D_{j \mid i}(y) \partial_{y} \psi_{j \mid i}^{\text {out }}(y, s)$. Inserting Eq. (B11) into Eq. (B10) and applying l'Hôpital's rule finally yields the Laplace transform of the probability density of the transition time

$$
\begin{aligned}
\tilde{\wp}_{j \mid i}^{\text {tr }}(s)= & \frac{J_{j \mid i}^{\text {out }}(0,0)}{J_{j \mid i}^{\text {out }}(0, s)} \frac{J_{j \mid i}^{\text {in }}(0, s) J_{j \mid i}^{\text {out }}(0, s)}{J_{j \mid i}^{\text {in }}(0,0) J_{j \mid i}^{\text {out }}(0,0)} \\
& \times \frac{J_{j \mid i}^{\text {in }}(0,0) \psi_{j \mid i}^{\text {out }}(0,0)}{J_{j \mid i}^{\text {in }}(0, s) \psi_{j \mid i}^{\text {out }}(0, s)} \times \frac{J_{j \mid i}^{\text {out }}\left(l_{j \mid i}, s\right)}{J_{j \mid i}^{\text {out }}\left(l_{j \mid i}, 0\right)} \\
= & \frac{\psi_{j \mid i}^{\text {out }}(0,0) J_{j \mid i}^{\text {out }}\left(l_{j \mid i}, s\right)}{\psi_{j \mid i}^{\text {out }}(0, s) J_{j \mid i}^{\text {out }}\left(l_{j \mid i}, 0\right)} .
\end{aligned}
$$

Since a function and its Laplace image are mapped one to one, Eq. (B12) fully characterizes the statistics of transition-path time. 


\section{Forward and backward symmetry of transition-path-time statistics}

The statistics of the corresponding backward transition can be determined in an analogous manner as Eq. (B12). Identifying $\psi_{i \mid j}^{\text {out }}(y, s)=\psi_{j \mid i}^{\text {in }}\left(l_{j \mid i}-y, s\right)$ and $J_{i \mid j}^{\text {out }}(y, s)=$ $-J_{j \mid i}^{\mathrm{in}}\left(l_{j \mid i}-y, s\right)$ the backward transition-path-time statistics $\tilde{\wp}_{j \mid i}^{\mathrm{tr}}(s)$ become

$$
\begin{aligned}
\tilde{\wp}_{i \mid j}^{\mathrm{tr}}(s) & =\frac{\psi_{j \mid i}^{\mathrm{in}}\left(l_{j \mid i}, 0\right) J_{j \mid i}^{\mathrm{in}}(0, s)}{\psi_{j \mid i}^{\text {in }}\left(l_{j \mid i}, s\right) J_{j \mid i}^{\text {in }}(0,0)} \\
& =\overbrace{w_{j \mid i}\left(l_{j \mid i}, 0\right) w_{j \mid i}(0, s)}^{w_{j \mid i}\left(l_{j \mid i}, s\right) w_{j \mid i}(0,0)} \frac{\psi_{j \mid i}^{\text {out }}(0,0) J_{j \mid i}^{\text {out }}\left(l_{j \mid i}, s\right)}{\psi_{j \mid i}^{\text {out }}(0, s) J_{j \mid i}^{\text {out }}\left(l_{j \mid i}, 0\right)} \\
& =\tilde{\wp}_{j \mid i}^{\mathrm{tr}}(s),
\end{aligned}
$$

where the first step follows from Eq. (B12), and in the second step we use Eq. (B8); in the last step, we identify $\tilde{\wp}_{j \mid i}^{\mathrm{tr}}(s)$ in Eq. (B12), which completes the proof of $\tilde{\wp}_{i \mid j}^{\mathrm{tr}}(s)=\tilde{\wp}_{j \mid i}^{\mathrm{tr}}(s)$. In other words, we hereby prove that the duration of both transitions $i \rightarrow j$ and $j \rightarrow i$ is identically distributed.

A similar derivation can be found in Ref. [57] for underdamped systems in which the momentum is assumed to be equilibrated.

\section{Dwell-time statistics obey a state symmetry}

Let $t$ denote the time of exiting from state $i$ toward state $j$ for the first time and $\delta t$ the corresponding transition-path time, which are distributed according to the probability densities $\wp_{j \mid i}^{\mathrm{loc}}(t)$ and $\wp_{j \mid i}^{\mathrm{tr}}(\delta t)$, respectively. The Laplace transform of $\wp_{j \mid i}^{\mathrm{loc}}(t)$ and $\wp_{j \mid i}^{\mathrm{tr}}(\delta t)$ is denoted by $\tilde{\wp}_{j \mid i}^{\text {loc }}(s)$ and $\tilde{\wp}_{j \mid i}^{\mathrm{tr}}(s)$. The transition-path-time statistics $\wp^{\mathrm{tr}}\left(\right.$ or $\left.\tilde{\wp}^{\mathrm{tr}}\right)$ do $n o t$ depend on the time at which a transition path begins and, hence, are independent of the time interval before $\tau=t-\tau$ that is called the dwell-time period. Therefore, we can obtain the statistics of the dwell time via deconvolution which in Laplace space becomes simple division

$$
\begin{aligned}
& \tilde{\wp}_{j \mid i}^{\text {dwell }}(s)=\frac{\tilde{\wp}_{j \mid i}^{\text {loc }}(s)}{\phi_{j \mid i}^{\text {loc }} \tilde{\wp}_{j \mid i}^{\text {tri }}(s)}
\end{aligned}
$$

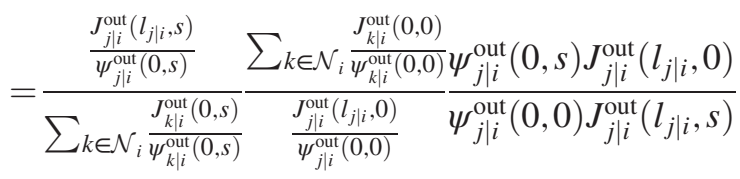

$$
\begin{aligned}
& =\frac{\sum_{k \in \mathcal{N}_{i}} J_{k \mid i}^{\text {out }}(0,0) / \psi_{k \mid i}^{\text {out }}(0,0)}{\sum_{k \in \mathcal{N}_{i}} J_{k \mid i}^{\text {out }}(0, s) / \psi_{k \mid i}^{\text {out }}(0, s)}=\tilde{\wp}_{i}^{\text {dwell }}(s),
\end{aligned}
$$

where in the second line we insert $\tilde{\wp}_{j \mid i}^{\text {loc }}(s)$ from Eq. (B5), $\phi_{j \mid i}^{\text {loc }}$ from Eq. (B6), and $\tilde{\wp}_{j \mid i}^{\mathrm{tr}}(s)$ from Eq. (B12); in the last line of Eq. (B14), we cancel equal terms in the numerator and denominator, respectively. Strikingly, we find that the result does not depend on the final state $j$, which is why the dwell-time statistics obeys a state symmetry, meaning that it depends only on the initial state $i$. Therefore, we can write $\tilde{\wp}_{j \mid i}^{\text {dwell }}(s)$ as $\tilde{\wp}_{i}^{\text {dwell }}(s)$ in the last step of Eq. (B14). Since the product $\tilde{\wp}_{j \mid i}^{\text {loc }}(s)=\phi_{j \mid i}^{\text {loc }} \tilde{\wp}_{j \mid i}^{\text {tr }}(s) \tilde{\wp}_{j \mid i}^{\text {dwell }}(s)$ in Laplace space becomes a convolution in the time domain, we hereby complete the proof of Eq. (3).

\section{Concluding remarks on the proofs}

To summarize this section, we show in Appendix B 1 that each change of state $i \rightarrow j$ in a network is taken with (splitting) probability $\phi_{j \mid i}^{\text {loc }}$ and has a corresponding distribution of residence time $t$ in turn being a sum of conditionally independent dwell time $\tau$ and transition-path time $\delta t=t-\tau$ (for a given transition between the pair of states $i \rightarrow j$ ). We prove two symmetries. First, we prove in Appendix B 4 that the statistics of transition-path time obeys a forward or backward symmetry $\wp_{j \mid i}^{\mathrm{tr}}(\delta t)=\wp_{i \mid j}^{\mathrm{tr}}(\delta t)$. Second, the statistics of dwell time is proven in Eq. (B14) to depend solely on the initial state $i$; that is, the dwell-time statistics does not depend on the state $j$ to which the trajectory finally transits.

\section{APPENDIX C: DECOMPOSITION OF PATHS}

\section{Generalized renewal theorem}

The classical renewal theorem [74] connects the firstpassage-time density to the propagator $P\left(a, T \mid i_{0}\right)$, which is the probability to find the network in state $i_{T}=a$ at time $T$ given that it was initially in state $i_{0}$. It can be understood as a decomposition of paths: Any system that starts at $i_{0}$ and arrives at $i_{T}=a$ at time $T$ must have reached $a$ for the first time at time $t \leq T\left(i_{t}=a\right)$ and then either stayed there or returned again after time $T-t$. In mathematical terms, this corresponds to [74]

$$
P\left(a, T \mid i_{0}\right)=\int_{0}^{T} d t \underbrace{P(a, T-t \mid a)}_{\text {return probability }} \overbrace{p_{a \mid i_{0}}(t)}^{\text {first passage } i_{0} \rightarrow a},
$$

where $p_{a \mid i_{0}}(t) d t$ denotes the probability that the process starting from $i_{0}$ reaches the position $i_{t^{\prime}}=a$ for the first time within the interval $t \leq t^{\prime} \leq t+d t$. We refer to $p_{a \mid i_{0}}(t)$ as the unconditioned first-passage-time density to the target state $a$ given that the system initially started from $i_{0}$. We call a first-passage problem "unconditioned" if there is just one target state $a$ as in Eq. (C1). The renewal theorem (C1) that connects the propagation of a system characterized by $P$ to unconditioned first-passage functionals embodied in the probability density $p_{a \mid i_{0}}(t)$ has been routinely used to study first-passage phenomena $[106,110]$. 
To study conditional first-passage problems [106] that involve more than a single-target state $a$, we need to generalize the renewal theorem $(\mathrm{C} 1)$ in the following way. Let us consider a set of target states $\mathcal{A}$ corresponding to a subset of all network states $\Omega=\{1, \ldots, N\}$, that is, $\mathcal{A} \subset \Omega$. A conditional first-passage problem asks for the first time until the system reaches the target state $a \in \mathcal{A}$ given that it has not yet visited any of the other target states from $\mathcal{A} \backslash\{a\}$. The problem is characterized by the joint density $\wp^{\mathcal{A}}{ }_{a \mid i_{0}}(t)$ to enter the set of target $\mathcal{A}$ for the first time at time $t$ and hitting the specific target $a \in \mathcal{A}$ upon starting from $i_{0}$, with normalization $\sum_{a \in \mathcal{A}} \int_{0}^{\infty} \wp^{\mathcal{A}}{ }_{a \mid i_{0}}(t) d t=1$. Note that the full set of neighboring states as targets $\mathcal{A}=$ $\mathcal{N}_{i_{0}}$ corresponds to $\wp^{\mathcal{A}} a \mid i_{0}=\wp_{a \mid i_{0}}^{\text {loc }}$ for all $a \in \mathcal{N}_{i_{0}}$. In the spirit of the classical renewal theorem (C1), we find that the conditional first-passage density to any subset $\mathcal{A}, \wp^{\mathcal{A}}$ is related to the simpler unconditioned first-passage-time densities according to

$$
\begin{aligned}
p_{a \mid i_{0}}(t) & =\wp^{\mathcal{A}} a_{a \mid i_{0}}(t)+\sum_{a^{\prime} \in \mathcal{A} \backslash\{a\}} \int_{0}^{t} p_{a \mid a^{\prime}}\left(t^{\prime}-t\right) \wp^{\mathcal{A}} a_{a^{\prime} \mid i_{0}}\left(t^{\prime}\right) d t^{\prime} \\
& \equiv \wp^{\mathcal{A}}{ }_{a \mid i_{0}}(t)+\sum_{a^{\prime} \in \mathcal{A} \backslash\{a\}} p_{a \mid a^{\prime}} * \wp^{\mathcal{A}}{ }_{a^{\prime} \mid i_{0}}(t), \quad(\mathrm{C} 2)
\end{aligned}
$$

which is a generalization of the renewal theorem to conditioned first-passage problems; in the last step, we introduce " $*$ " as the one-sided convolution operation. An illustration of the generalized renewal theorem for a network with five states $\Omega=\{1,2,3,4,5\}$ and two target states $\mathcal{A}=\{1,2\}$ with initial condition $i_{0}=3$ is shown in Fig. 9. In the simplest case, when the subset $\mathcal{A}$ contains a single element $\mathcal{A}=\{a\}$, we consistently obtain $p_{a \mid i_{0}}(t)=\wp_{a \mid i_{0}}^{\{a\}}(t)$; i.e., the conditioned first-passage problem becomes a unconditioned one. Laplace transforming the renewal theorem $(\mathrm{C} 2)$, where the Laplace transform of some generic function $f(t)$ is defined as $\tilde{f}(s)=\int_{0}^{\infty} e^{-s t} f(t) d t$ (tacitly assuming that all functions are of exponential order), we obtain

$$
\tilde{p}_{a \mid i_{0}}(s)=\tilde{\wp}^{\mathcal{A}}{ }_{a \mid i_{0}}(s)+\sum_{a^{\prime} \in \mathcal{A} \backslash\{a\}} \tilde{p}_{a \mid a^{\prime}}(s) \tilde{\wp}^{\mathcal{A}}{ }_{a^{\prime} \mid i_{0}}(s),
$$

where the convolution in the last term of Eq. (C2) becomes a product after the Laplace transform. It is worth mentioning that Eq. (C3) via Eq. (25) links diffusion to (unconditioned) first-passage statistics of currents as studied in Ref. [111] (see also Refs. [112-114]).

In the Supplemental Material Sec. I.A [75], we show how the generalized renewal theorem Eq. (C3) can be used to deduce explicit conditional moments of first-passage time, which correspond to a multitarget search problem, in terms of simpler unconditioned "single-target" quantities. As we explain below (see Appendix C 3) one can in fact construct any network problem by solving for networks with a specific and simpler starlike topology. This sequential strategy, which we explain in the following, allows for a systematic study of general networks.

\section{Renewal theorem on starlike graphs}

Let us for now focus on graphs with a starlike topology, where all $n=N-1$ "outer nodes" are target states, that is, $\mathcal{A}=\{1,2, \ldots, n\}$, and the starting node is the "inner state" $i_{0}=N$ as depicted in Fig. 10. In the case of a starlike topology (i.e., $\mathcal{A}=\mathcal{N}_{i_{0}}$ and $\wp^{\mathcal{A}}{ }_{a \mid i_{0}}=\wp_{a \mid i_{0}}^{\text {loc }}$ ), the renewal theorem Eq. (C3) simplifies, meaning that it can be inverted more easily.

In a first crucial step, we realize that each path on a starlike graph, which starts from one end of the star $a$ to another end $a^{\prime} \neq a$, must pass through the center $i_{0}$. That is, the unconditioned first-passage time from $a$ to $a^{\prime}$ is the sum of first-passage time from $a^{\prime}$ to $i_{0}$ and the first-passage time from $i_{0}$ to $a$, which effectively implies $\tilde{p}_{a \mid a^{\prime}}(s)=$ $\tilde{p}_{a \mid i_{0}}(s) \tilde{p}_{i_{0} \mid a^{\prime}}(s)$ [see Fig. 10(c)]. Using $\tilde{p}_{a \mid a^{\prime}}(s)=\tilde{p}_{a \mid i_{0}}(s) \times$ $\tilde{p}_{i_{0} \mid a^{\prime}}(s)$ for $a^{\prime} \neq a$, the renewal theorem (C3) in matrix form becomes

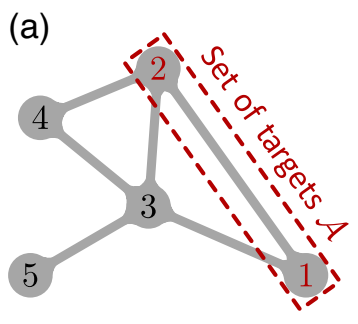

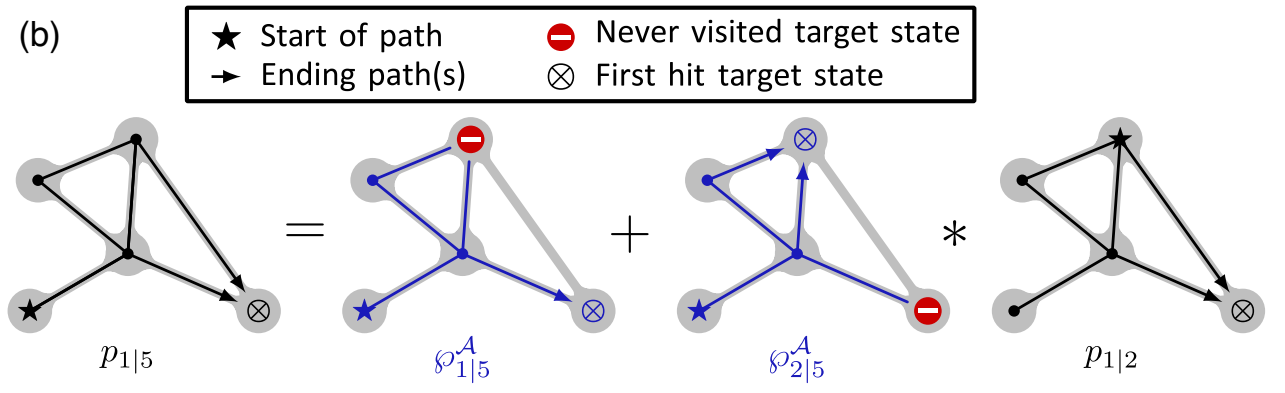

FIG. 9. Illustration of generalized renewal theorem. (a) Network with five states $\Omega=\{1,2,3,4,5\}$. The dashed box encloses one set of target state $\mathcal{A}$, which is here chosen to be $\mathcal{A}=\{1,2\}$. The network corresponds to the subnetwork from Fig. 1(e) in the main text (see also Fig. 3 therein). (b) Path decomposition according to generalized renewal theorem from Eq. (C2) or equivalently Eq. (C3) for the special case of two target states $\mathcal{A}=\{1,2\}$ with $a=1$. Each arrow tip corresponds to a path ending at the particular state without having visited that state before; each filled dot is a state that may have been visited. 
(a)

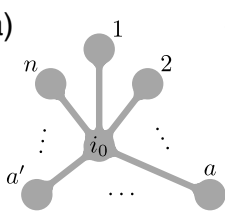

(b)

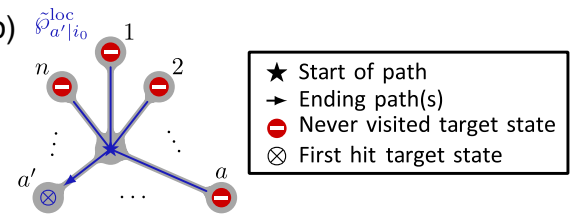

(c)

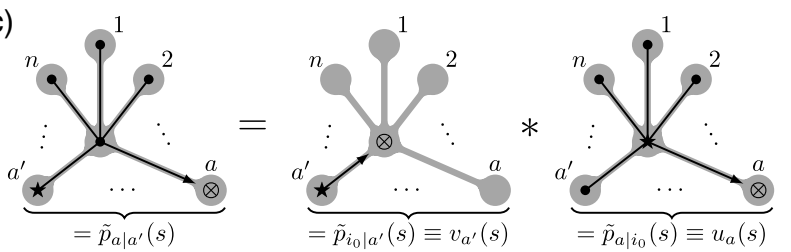

FIG. 10. Starlike graph. (a) Starlike graph with $N$ states from which $n=N-1$ are "outer states" $a, a^{\prime}=1, \ldots, n$ and one state is called the "inner state" $N$. (b) Conditional first-passage paths belonging to $\tilde{\wp}_{a^{\prime} \mid i_{0}}^{\text {loc }}(s)$. (c) Illustration of $\tilde{p}_{a \mid a^{\prime}}(s)=\tilde{p}_{a \mid i_{0}}(s) \times$ $\tilde{p}_{i_{0} \mid a^{\prime}}(s)$, which holds for $a^{\prime} \neq a$ and effectively means that each path starting from $a^{\prime}$ must pass through the center $i_{0}$ to reach the other end $a\left(a \neq a^{\prime}\right)$. The matrix $\mathcal{D}(s)$ in Eq. (C4) accounts and corrects for $\tilde{p}_{a \mid a}(s)=1 \neq \tilde{p}_{a \mid i_{0}}(s) \tilde{p}_{i_{0} \mid a}(s)$.

$$
u_{a}(s)=\sum_{a^{\prime}=1}^{n}\left[\mathcal{D}(s)+\boldsymbol{u}(s) \boldsymbol{v}(s)^{\top}\right]_{a a^{\prime}} \tilde{\wp}_{a^{\prime} \mid i_{0}}^{\mathrm{loc}}(s),
$$

where $\boldsymbol{u}(s)$ and $\boldsymbol{v}(s)$ are vectors with elements $u_{a}(s) \equiv$ $\tilde{p}_{a \mid i_{0}}(s)$ and $v_{a}(s) \equiv \tilde{p}_{i_{0} \mid a}(s)$, respectively, and $\mathcal{D}(s)$ denotes a diagonal matrix with elements $\mathcal{D}_{i i}(s)=1-$ $u_{i}(s) v_{i}(s)$, which corrects for $\tilde{p}_{a \mid a}(s)=1 \neq \tilde{p}_{a \mid i_{0}}(s) \times$ $\tilde{p}_{i_{0} \mid a}(s)$. Using the Sherman-Morrison-Woodbury formula, we are able to invert the matrix $\mathcal{D}+\boldsymbol{u} \boldsymbol{v}^{\top}$ to get

$$
\begin{aligned}
\tilde{\wp}_{a \mid i_{0}}^{\mathrm{loc}}(s) & =\frac{\frac{u_{a}(s)}{1-u_{a}(s) v_{a}(s)}}{1-n+\sum_{a^{\prime}}\left[1-u_{a^{\prime}}(s) v_{a^{\prime}}(s)\right]^{-1}} \\
& =\frac{\frac{s u_{a}(s)}{1-u_{a}(s) v_{a}(s)}}{(1-n) s+\sum_{a^{\prime}} \frac{s}{1-u_{a^{\prime}}(s) v_{a^{\prime}}(s)}},
\end{aligned}
$$

which is the central result of this subsection that allows us to obtain conditional many-target first-passage-time distributions from simpler unconditioned single-target firstpassage-time densities. The local splitting probability, which formally reads $\phi_{a \mid i_{0}}^{\text {loc }}=\tilde{\wp}_{a \mid i_{0}}^{\text {loc }}(0)$, can be obtained by taking the limit $s \rightarrow 0$.

In the Supplemental Material Sec. I.B [75], we show how Eq. (C5) can be used to express the splitting probability $\phi_{a||_{0}}^{\text {loc }}$, the conditional mean first passage $\langle t\rangle_{a \mid i_{0}}^{\text {loc }}$, and the second moment of exit time $\left\langle t^{2}\right\rangle_{i_{0}}^{\text {exit }}=\sum_{a} \phi_{a \mid i_{0}}^{\text {loc }}\left\langle t^{2}\right\rangle_{a \mid i_{0}}^{\text {loc }}$, merely in terms of simpler first and second moments of unconditioned first-passage time $\langle t\rangle_{j \mid i}^{\text {single }}=-\left.\partial_{s} \tilde{p}_{j \mid i}(s)\right|_{s=0}$, $\left\langle t^{2}\right\rangle_{j \mid i}^{\text {single }}=\left.\partial_{s}^{2} \tilde{p}_{j \mid i}(s)\right|_{s=0}$ with only a single-target state. The results for $\phi_{a \mid i_{0}}^{\text {loc }},\langle t\rangle_{a||_{0}}^{\text {loc }}$, and $\left\langle t^{2}\right\rangle_{i_{0}}^{\text {exit }}$ in terms of

unconditioned moments of first-passage times are displayed in the Supplemental Material Sec. I. B [75] and can be used to derive the main practical result (see Sec. II D) after some quite tedious calculations, which are carried out as follows.

First, we derive all first and second moments of the unconditioned first-passage time alongside the first two moments of transition time (see Supplemental Material Sec. II. D [75]). Second, we insert them into the expressions for $\phi_{a \mid i_{0}}^{\text {loc }},\langle t\rangle_{a||_{0}}^{\text {loc }}$, and $\left\langle t^{2}\right\rangle_{i_{0}}^{\text {exit }}$ listed in the Supplemental Material [75] [see Eqs. (S15), (S16), and (S19) therein], which is carried out in the Supplemental Material Sec. III [75]. This fully proves the results in Sec. II D.

\section{Networks with general topology from starlike subgraphs}

The simplest network topology is a starlike topology, for which we are able to conveniently express moments of conditional first-passage times in terms of simple unconditioned first-passage moments as explained in the Supplemental Material Sec. I.B [75]. In the following, we show that according to Ref. [37] (see also Refs. [3840]), each network can be decomposed exactly into a full set of subnetworks with a starlike topology. Thereby, each starlike subgraph characterizes the local kinetics on a graph in the vicinity of a network state. Hence, we use all starlike subgraphs as building blocks to build and describe a general network.

Suppose that we are dealing with a large-scale network with a set of $N$ states, such that for each state $i \in \Omega=$ $\{1, \ldots, N\}$, there exists a nonempty set of neighboring states $\mathcal{N}_{i} \subset \Omega$ with $i \notin \mathcal{N}_{i}$. A fully connected network corresponds to $\mathcal{N}_{i}=\{1, \ldots, i-1, i+1, \ldots, N\}$. For the five-state network in Fig. 11, all sets of neighboring states are $\mathcal{N}_{1}=\{2,3\}, \quad \mathcal{N}_{2}=\{1,3,4\}, \quad \mathcal{N}_{3}=\{1,2,4,5\}$, $\mathcal{N}_{4}=\{2,3\}$, and $\mathcal{N}_{5}=\{3\}$. The probability density that starting from state $i$ a nearest-neighboring state $j \in \mathcal{N}_{i}$ will be reached for the first time at time $t$ is distributed according to the probability density $\wp_{j \mid i}^{\text {loc }}(t)$ with a Laplace transform $\tilde{\wp}_{j \mid i}^{\text {loc }}(s)$, where $\phi_{j \mid i}^{\text {loc }}=\tilde{\wp}_{j \mid i}^{\text {loc }}(0)=\int_{0}^{\infty} \wp_{j \mid i}^{\text {loc }}(t) d t$ is the (splitting) probability that starting from $i$ the next visited state will be $j$. We define the matrix $\tilde{\mathbb{P}}(s)$ as

$$
\tilde{\mathbb{P}}(s)_{i j}= \begin{cases}\tilde{\wp}_{i \mid j}^{\text {loc }}(s) & \text { if } i \in \mathcal{N}_{j}, \\ 0 & \text { otherwise. }\end{cases}
$$

Note that $\tilde{\mathbb{P}}(s)$ is a hollow matrix, since $\tilde{\mathbb{P}}(s)_{i i}=0$. Taylor expanding Eq. (C6), we obtain

$$
\tilde{\mathbb{P}}(s)=\boldsymbol{\Phi}-s \mathcal{T}+O(s)^{2},
$$

where $\boldsymbol{\Phi}_{i j}=\phi_{i \mid j}^{\text {loc }}$ and $\mathcal{T}_{i j}=\phi_{i \mid j}^{\text {loc }}\langle t\rangle_{i \mid j}^{\text {loc }}$ for $i \in \mathcal{N}_{j}$ and $j=1, \ldots, N$. We emphasize that working in Laplace space 
(a)

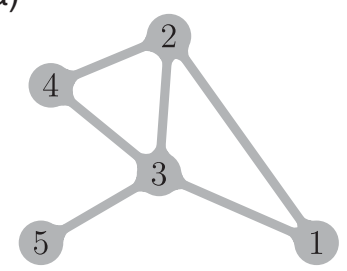

(b)

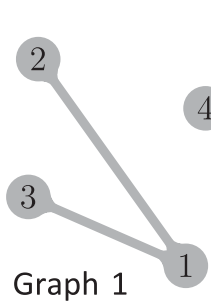

Graph 2

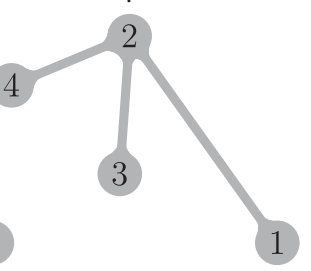

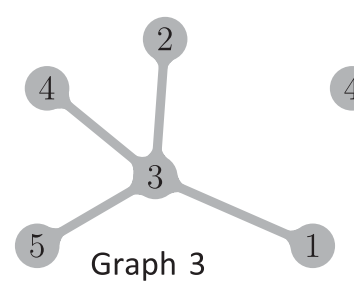

Graph 4

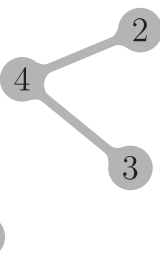

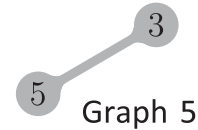

FIG. 11. Network decomposition into starlike subgraphs. (a) Full graph with five network states. (b) All five starlike subgraphs. The set of neighboring states contains $\mathcal{N}_{1}=\{2,3\}, \mathcal{N}_{2}=\{1,3,4\}, \mathcal{N}_{3}=\{1,2,4,5\}, \mathcal{N}_{4}=\{2,3\}$, and $\mathcal{N}_{5}=\{3\}$.

allows us to conveniently add independent random variables. Namely, for any two independent random variables $t_{1}$ and $t_{2}$ distributed according to the densities $f_{1}$ and $f_{2}$ with Laplace transforms $\tilde{f}_{i}(s)=\int_{0}^{\infty} f_{i}(t) e^{-s t} d t \equiv\left\langle e^{-s t_{i}}\right\rangle$, we have that $\tilde{f}_{1+2}(s)=\left\langle e^{-s\left(t_{1}+t_{2}\right)}\right\rangle=\left\langle e^{-s t_{1}}\right\rangle\left\langle e^{-s t_{2}}\right\rangle=\tilde{f}_{1}(s) \tilde{f}_{2}(s)$. That is, in Laplace space the sum of random variables is reflected by the product of the Laplace transforms of the corresponding probability densities [see also the last terms in the generalized renewal theorem Eqs. (C2) and (C3)]. Conversely, a plain product in Laplace space becomes a convolution in the time domain $f_{1+2}(t)=f_{1} * f_{2}(t)$.

Having established the local kinetics, we can now determine the first-passage time to a set of target states $\mathcal{A}$ starting from state $i_{0} \notin \mathcal{A}\left(i_{0} \in \Omega \backslash \mathcal{A}\right)$ for a general network as follows. To select a target state and remaining states, we first define the projection matrix onto target state $\mathcal{A}$ and the rest, i.e., $\mathcal{A}^{c} \equiv \Omega \backslash \mathcal{A}$, which are given by

$\mathbf{1}_{\mathcal{A}} \equiv \sum_{i \in \mathcal{A}}|i\rangle\langle i| \quad$ and $\quad \mathbf{1}_{\mathcal{A}^{c}} \equiv \sum_{i \notin \mathcal{A}}|i\rangle\langle i|=\mathbf{1}-\mathbf{1}_{\mathcal{A}}$,

respectively, where $\langle i|=| i\rangle^{\top}$ is a unit column vector with all elements zero except the $i$ th component and 1 is the identity matrix. The matrices $\mathbf{1}_{\mathcal{A}}$ and $\mathbf{1}_{\mathcal{A}^{c}}$ are the indicator functions of $\mathcal{A}$ and $\mathcal{A}^{c}$, respectively. For example, for all target states $\alpha \in \mathcal{A}$, we find $\mathbf{1}_{\mathcal{A}}|\alpha\rangle=|\alpha\rangle$ and $\mathbf{1}_{\mathcal{A}^{c}}|\alpha\rangle=0$, whereas for all remaining states $\beta \in \mathcal{A}^{c}$, we have $\mathbf{1}_{\mathcal{A}}|\beta\rangle=$ 0 and $\mathbf{1}_{\mathcal{A}^{c}}|\beta\rangle=|\beta\rangle$. Starting from $i_{0} \in \mathcal{A}^{c}$, the Laplace transform of the probability density to hit the target state $a \in \mathcal{A}$ "after the first step" (without having visited any of the remaining states) is given by $\left\langle a\left|\mathcal{Q}^{(1)}(s)\right| i_{0}\right\rangle=$ $\left\langle a|\tilde{\mathbb{P}}(s)| i_{0}\right\rangle$. Similarly, if we select all elements that perform exactly one jump into a state $j \in \mathcal{A}^{c}$ and then enter $a$ in the second jump, we obtain $\langle a|\tilde{\mathbb{P}}(s)| j\rangle\left\langle j|\tilde{\mathbb{P}}(s)| i_{0}\right\rangle$, which after summing over all intermediate nontarget states with Eq. (C8), yields $\left\langle a\left|\mathcal{Q}^{(2)}(s)\right| i_{0}\right\rangle=\left\langle a\left|\tilde{\mathbb{P}}(s) \mathbf{1}_{\mathcal{A}^{c}} \tilde{\mathbb{P}}(s)\right| i_{0}\right\rangle$. More generally, the Laplace transform of the probability density to hit target $a$ for the first time exactly after $k$ th transitions while transiting $k-1$ times between nontarget states is given by

$$
\left\langle a\left|\mathcal{Q}^{(k)}\right| i_{0}\right\rangle=\left\langle a\left|\tilde{\mathbb{P}}(s)\left[\mathbf{1}_{\mathcal{A}^{c}} \tilde{\mathbb{P}}(s)\right]^{k-1}\right| i_{0}\right\rangle .
$$

Summing now over all possible numbers of intermediate transitions, we obtain a geometric sum that yields [37]

$\tilde{\wp}^{\mathcal{A}}{ }_{a \mid i_{0}}(s) \equiv \sum_{k=1}^{\infty}\left\langle a\left|\mathcal{Q}^{(k)}\right| i_{0}\right\rangle=\left\langle a\left|\tilde{\mathbb{P}}(s)\left[\mathbf{1}-\mathbf{1}_{\mathcal{A}^{c}} \tilde{\mathbb{P}}(s)\right]^{-1}\right| i_{0}\right\rangle$,

which is the main result of this subsection. This result allows us to express conditional first-passage times toward any set of targets $\mathcal{A}$ in terms of the local first-passage-time densities. The inverse Laplace transform $(s \rightarrow t)$ of Eq. (C10) yields the joint probability density $\wp^{\mathcal{A}}{ }_{a \mid i_{0}}(t)$ that the continuous trajectory starting from node $i_{0} \in \Omega \backslash \mathcal{A}=$ $\mathcal{A}_{c}$ arrives at time $t$ for the first time in state $a \in \mathcal{A}$ without having visited any other state within $\mathcal{A}$. The probability is normalized according to $\sum_{a \in \mathcal{A}} \int_{0}^{\infty} \wp^{\mathcal{A}}{ }_{a \mid i_{0}}(t) d t=1$. The case in which $\mathcal{A}$ contains all neighbors of $i_{0}$, that is, $\mathcal{A}=\mathcal{N}_{i_{0}}$, one immediately obtains $\mathbf{1}_{\mathcal{A}^{c}} \tilde{\mathbb{P}}(s)\left|i_{0}\right\rangle=0$, which simplifies Eq. (C10) to $\tilde{\wp}^{\mathcal{A}}{ }_{a \mid i_{0}}=\left\langle a|\tilde{\mathbb{P}}(s)| i_{0}\right\rangle=\tilde{\wp}_{a \mid i_{0}}^{\text {loc }}(s)$ for all $a \in \mathcal{A}=\mathcal{N}_{i_{0}}$. The independence between transition time and dwell time Eq. (3) allows us to express nonzero elements of the hollow matrix $\tilde{\mathbb{P}}(s)$ in the form of the product $\tilde{\mathbb{P}}(s)_{j i} \equiv \tilde{\wp}_{j \mid i}^{\text {loc }}(s)=\phi_{j \mid i}^{\text {loc }} \tilde{\wp}_{j \mid i}^{\text {tr }}(s) \tilde{\wp}_{i}^{\text {dwell }}(s)$ for any pair $i, j \neq i$.

From Eq. (C10) follows the splitting probability, i.e., the probability to reach $a$ from $i_{0}$ before reaching any other state within $\mathcal{A}$ which reads

$$
\begin{aligned}
\phi_{a \mid i_{0}}^{\mathcal{A}} & \equiv \int_{0}^{\infty} \wp^{\mathcal{A}}{ }_{a \mid i_{0}}(t) d t=\left.\tilde{\wp}^{\mathcal{A}}{ }_{a \mid i_{0}}(s)\right|_{s=0} \\
& =\left\langle a\left|\boldsymbol{\Phi}\left(\mathbf{1}-\mathbf{1}_{\mathcal{A}^{c}} \mathbf{\Phi}\right)^{-1}\right| i_{0}\right\rangle,
\end{aligned}
$$

where we use $\boldsymbol{\Phi}_{i j}=\phi_{i \mid j}^{\text {loc }}$ from Eq. (C7). Inserting the matrix $\mathcal{T}_{i j}=\phi_{i \mid j}^{\text {loc }}\langle t\rangle_{i \mid j}^{\mathrm{loc}}$ from Eq. (C7) the mean firstpassage time from $i_{0}$ to $a$ conditioned not to visit any state $j \in \mathcal{A} \backslash\{a\}$ in turn reads

$$
\langle t\rangle_{a \mid i_{0}}^{\mathcal{A}}=\left\langle a\left|\left(\mathbf{1}-\boldsymbol{\Phi} \mathbf{1}_{\mathcal{A}^{c}}\right)^{-1} \mathcal{T}\left(\mathbf{1}-\mathbf{1}_{\mathcal{A}^{c}} \boldsymbol{\Phi}\right)^{-1}\right| i_{0}\right\rangle / \phi_{a \mid i_{0}}^{\mathcal{A}} .
$$


A few remarks are in order before we prove Eq. (C12). Equations (9)-(13) render Eqs. (C11) and (C12) fully explicit. As an illustration, in Appendix C 4 we apply Eqs. (C11) and (C12) to the synthetic network used in Fig. 3, where a diffusive barrier along link 1-3 mimics the effect of an entropic bottleneck [115] (details about the model are given in Appendix E1).

The conditional mean first-passage time $(\mathrm{C} 12)$ follows from

$$
\begin{aligned}
\phi^{\mathcal{A}}{ }_{a \mid i_{0}}\langle t\rangle^{\mathcal{A}}{ }_{a \mid i_{0}} \\
\equiv \int_{0}^{\infty} \wp^{\mathcal{A}}{ }_{a \mid i_{0}}(t) t d t=-\left.\frac{\partial}{\partial s} \tilde{\wp}^{\mathcal{A}}{ }_{a \mid i_{0}}(s)\right|_{s=0} \\
=\left\langle a\left|\mathcal{T}\left(\mathbf{1}-\mathbf{1}_{\mathcal{A}^{c}} \boldsymbol{\Phi}\right)^{-1}\right| i_{0}\right\rangle \\
\quad+\left\langle a\left|\boldsymbol{\Phi}\left(\mathbf{1}-\mathbf{1}_{\mathcal{A}^{c}} \boldsymbol{\Phi}\right)^{-1} \mathbf{1}_{\mathcal{A}^{c}} \mathcal{T}\left(\mathbf{1}-\mathbf{1}_{\mathcal{A}^{c}} \boldsymbol{\Phi}\right)^{-1}\right| i_{0}\right\rangle \\
=\left\langle a\left|\left[\mathbf{1}+\boldsymbol{\Phi} \mathbf{1}_{\mathcal{A}^{c}}\left(\mathbf{1}-\boldsymbol{\Phi} \mathbf{1}_{\mathcal{A}^{c}}\right)^{-1}\right] \mathcal{T}\left(\mathbf{1}-\mathbf{1}_{\mathcal{A}^{c}} \boldsymbol{\Phi}\right)^{-1}\right| i_{0}\right\rangle \\
=\left\langle a\left|\left(\mathbf{1}-\boldsymbol{\Phi} \mathbf{1}_{\mathcal{A}^{c}}\right)^{-1} \mathcal{T}\left(\mathbf{1}-\mathbf{1}_{\mathcal{A}^{c}} \boldsymbol{\Phi}\right)^{-1}\right| i_{0}\right\rangle,
\end{aligned}
$$

where we use the product rule of differentiation " $\partial(f g)=(\partial f) g+f \partial g "$ and the formula $(d / d s) \mathbf{A}^{-1}=$ $-\boldsymbol{A}^{-1}(d \boldsymbol{A} / d s) \boldsymbol{A}^{-1}$ in the third line, and $\mathbf{1}_{\mathcal{A}^{c}}\left(\mathbf{1}-\boldsymbol{\Phi} \mathbf{1}_{\mathcal{A}^{c}}\right)^{-1}=$ $\left(\mathbf{1}-\mathbf{1}_{\mathcal{A}^{c}} \boldsymbol{\Phi}\right)^{-1} \mathbf{1}_{\mathcal{A}^{c}}$ in the second to last line, which finally leads to Eq. (C12). Note that the conditional mean first-passage time $\langle t\rangle^{\mathcal{A}} a \mid i_{0} \equiv\left[\int_{0}^{\infty} \wp^{\mathcal{A}}{ }_{a \mid i_{0}}(t) t d t\right] /$ $\left[\int_{0}^{\infty} \wp^{\mathcal{A}} a \mid i_{0}(t) d t\right]$ is obtained by dividing Eq. (C13) by the splitting probability Eq. (C11). This completes the proof of Eq. (C12).

Higher moments can formally be obtained along the same lines via Eq. (C10), such that the $k$ th moment satisfies

$\phi_{a \mid i_{0}}^{\mathcal{A}}\left\langle t^{k}\right\rangle_{a \mid i_{0}}^{\mathcal{A}} \equiv \int_{0}^{\infty} \wp^{\mathcal{A}}{ }_{a \mid i_{0}}(t) t^{k} d t=\left.(-1)^{k} \frac{\partial^{k}}{\partial s^{k}} \tilde{\wp}^{\mathcal{A}}{ }_{a \mid i_{0}}(s)\right|_{s=0}$.

Using Eq. (C10), we can effectively deduce any moment of the first-passage time within the network from $\tilde{\mathbb{P}}(s)$.

If the network can be described by memoryless jump dynamics [6] as, for instance, in the celebrated Gillespie algorithm [1,2], the transitions between network states are characterized by constant transition rates $w_{i \rightarrow j}$ from state $i$ to state $j$. In this case, the time until the state changes is exponentially distributed with the rate of leaving state $i$, $r_{i}=\sum_{j \in \mathcal{N}_{i}} w_{i \rightarrow j}$, yielding the same exit-time distribution $\wp_{i}^{\text {exit }}(t)=\sum_{k} \mathbb{P}(t)_{k i}^{M}=r_{i} e^{-r_{i} t}$ irrespective of the final state $j$, with probability $\phi_{j \mid i}^{\text {loc }}=w_{i \rightarrow j} / r_{i}$, i.e., $\wp_{j \mid i}^{\text {loc }}(t) /$ $\phi_{j \mid i}^{\text {loc }}=\wp_{i}^{\text {exit }}(t)$. A Laplace transform $t \rightarrow s$ of such memoryless kinetics would yield $\tilde{\mathbb{P}}(s)_{j \mid i}^{M}=\phi_{j \mid i}^{\text {loc }} r_{i}\left(s+r_{i}\right)^{-1}$ along with the $k$ th moment $\left\langle t^{k}\right\rangle_{j \mid i}^{\text {loc }}=\left\langle\tau^{k}\right\rangle_{i}^{\text {dwell }}=k ! r_{i}^{-k}$.

\section{Fingerprints of memory}

Unique fingerprints of memory in state-to-state kinetics emerge already under minimal assumptions. Consider the kinetics from state $i_{0}=3$ to the pair of target states $\mathcal{A}=$ $\{1,2\}$ in the network depicted in Fig. 9(a) [see also the trajectory in Figs. 3(a) and 6], which corresponds to

$$
\mathbf{1}_{\mathcal{A}}=\left(\begin{array}{ccccc}
1 & 0 & 0 & 0 & 0 \\
0 & 1 & 0 & 0 & 0 \\
0 & 0 & 0 & 0 & 0 \\
0 & 0 & 0 & 0 & 0 \\
0 & 0 & 0 & 0 & 0
\end{array}\right), \quad \mathbf{1}_{\mathcal{A}^{c}}=\left(\begin{array}{ccccc}
0 & 0 & 0 & 0 & 0 \\
0 & 0 & 0 & 0 & 0 \\
0 & 0 & 1 & 0 & 0 \\
0 & 0 & 0 & 1 & 0 \\
0 & 0 & 0 & 0 & 1
\end{array}\right) .
$$

In order to infer the waiting-time distribution $\wp^{\text {loc }}$ for the exit from all states, respectively, we simulate $4 \times 10^{5}$ exits from each state (see Fig. 14 in Appendix E 1). The normalized waiting-time distribution for the exit from state 3 is genuinely heterogeneous [98]; i.e., it shows strong variations between the respective legs [see Fig. 3(c) and see Fig. 14 for a more detailed analysis of the complete network]. The splitting probability and the local mean waiting time are given by

$$
\begin{aligned}
\boldsymbol{\Phi} & \approx\left(\begin{array}{ccccc}
0 & 0.33 & 0.25 & 0 & 0 \\
0.5 & 0 & 0.25 & 0.5 & 0 \\
0.5 & 0.33 & 0 & 0.5 & 1 \\
0 & 0.33 & 0.25 & 0 & 0 \\
0 & 0 & 0.25 & 0 & 0
\end{array}\right), \\
\mathcal{T} & \approx\left(\begin{array}{ccccc}
0 & 0.44 & 4.02 & 0 & 0 \\
5.64 & 0 & 1.58 & 0.66 & 0 \\
10.52 & 0.44 & 0 & 0.66 & 1.33 \\
0 & 0.44 & 1.58 & 0 & 0 \\
0 & 0 & 1.58 & 0 & 0
\end{array}\right) .
\end{aligned}
$$

One can confirm that the system satisfies detailed balance, since $\ln \left(\boldsymbol{\Phi}_{j i} / \boldsymbol{\Phi}_{i j}\right)=\ln \left|\mathcal{N}_{j}\right|-\ln \left|\mathcal{N}_{i}\right|$ holds, where $\left|\mathcal{N}_{i}\right|$ is the number of states adjacent to $i$.

We now inspect the probability to reach the target state 1 (2) within $\mathcal{A}$ before reaching state 2 (1). Note that a trajectory may reach state 1 through the link $1-3$ or via state 4. Such conditioned transition kinetics quantify nonlocal effects and are particularly important for marginal observations; i.e., when we do not monitor all states but instead only a subset (in this case, states 1-3) while the remaining states are left as part of the "heat bath." This scenario is very relevant from an experimental point of view, since we can typically monitor only a limited number of states.

After inserting Eqs. (C15) and (C16) into Eqs. (C11) and (C12), respectively, we find $\phi_{1 \mid 3}^{\mathcal{A}}=0.4$ and $\phi_{2 \mid 3}^{\mathcal{A}}=0.6$ 
while $\langle t\rangle_{1 \mid 3}^{\mathcal{A}} \approx 8.26,\langle t\rangle_{2 \mid 3}^{\mathcal{A}} \approx 6.80$. A Markov process would presume isotropic mean waiting times $\mathcal{T}_{i j}^{M}=\phi_{i \mid j}\langle t\rangle_{j}^{\text {exit }}$, which yields $\langle t\rangle_{1 \mid 3}^{\mathcal{A}, M} \approx 5.92<\langle t\rangle_{2 \mid 3}^{\mathcal{A}, M} \approx 9.14$, whereas $\langle t\rangle_{1 \mid 3}^{\mathcal{A}} \approx 8.26>\langle t\rangle_{2 \mid 3}^{\mathcal{A}} \approx 6.80$.

\section{APPENDIX D: THERMODYNAMIC CONSISTENCY OF COARSE GRAINING AND DISCONTINUOUS FORCE FIELDS}

In this Appendix, we connect the thermodynamic consistency of the coarse graining to the preservation of cycles [55], which implies that the entropy production rate $[6,86]$ is preserved in the coarse-grained process as discussed in Sec. III B. This underlines that the violation of detailed balance is entirely encoded in the splitting probabilities (see also Ref. [53]). In addition, we derive the special limit of local detailed balance in the presence of a timescale separation. Finally, we consider discontinuous diffusion coefficients and/or force fields.

\section{Thermodynamic consistency follows from the preservation of cycle affinities}

Whether or not the system relaxes to an equilibrium distribution is entirely encoded in the microscopic force field $\boldsymbol{F}$. If the force field is conservative, that is, $\boldsymbol{F}(\boldsymbol{x})=-\nabla U(\boldsymbol{x})$, the resulting stationary state corresponds to thermodynamic equilibrium. Detailed balance is said to be broken if the time-independent force field has a nonzero rotation, or equivalently, if at least one directed cycle $\mathcal{C}$ (see thick arrows in Fig. 12) exists, for which the integral

$$
\mathcal{A}[\mathcal{C}] \equiv \oint_{\mathcal{C}} \boldsymbol{F}(\boldsymbol{x}) \cdot d x=\sum_{\alpha=1}^{\nu} \int_{i_{\alpha}^{\mathcal{C}}}^{i_{\alpha+1}^{\mathcal{C}}} \boldsymbol{F}(\boldsymbol{x}) \cdot d x
$$

called affinity [86] is nonzero. If we insert the first main result, Eq. (2), into Eq. (D1), we obtain

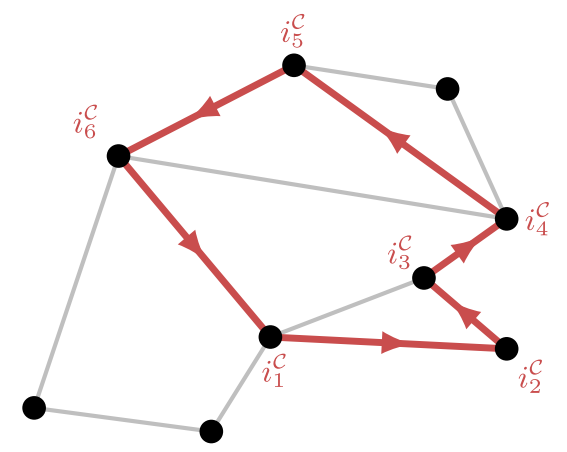

FIG. 12. One directed cycle $\mathcal{C}=i_{1}^{\mathcal{C}} \rightarrow i_{2}^{\mathcal{C}} \rightarrow, \ldots, \rightarrow i_{\nu}^{\mathcal{C}} \rightarrow$ $i_{\nu+1}^{\mathcal{C}}=i_{1}^{\mathcal{C}}$ (see thick red arrow lines) is highlighted which encloses $\nu=|\mathcal{C}|=6$ nodes within a total set of $\Omega=9$ nodes.

$$
\mathcal{A}[\mathcal{C}]=\sum_{\alpha=1}^{\nu} \ln \left[\frac{\phi_{i_{\alpha+1}^{c}}^{\mathrm{loc}} \mid i_{\alpha}^{\mathcal{C}}}{\phi_{i_{\alpha}^{\prime} \mid i_{\alpha+1}^{c}}^{\mathrm{loc}}}\right],
$$

where we use the fact that the terms involving the function " $g$ " form a vanishing telescope sum. Equation (D2) relates the affinity of all cycles $\mathcal{C}$ exactly to the splitting probabilities $\left\{\phi_{j \mid i}^{\text {loc }}\right\}$. Therefore, detailed balance is satisfied as soon as all closed cycles in a network satisfy $\mathcal{A}[\mathcal{C}]=0$. This proves that the splitting probabilities alone encode the breaking or validity of detailed balance.

Because of the preservation of cycle affinities [see Eqs. (D1) and (D2)], the steady-state entropy production is entirely encoded in the splitting probabilities $\left\{\phi_{j \mid i}^{\text {loc }}\right\}$. This can be understood as an alternative proof of the preservation of the entropy production shown in Sec. III B (see also Ref. [55]).

\section{The peculiar local equilibration}

Let us now address the limit of a timescale separation that leads to a local equilibration prior to any change of state. In the limit of high free-energy barriers (i.e., $B_{j \mid i} \rightarrow$ $\infty$ in Fig. 5), the first two auxiliary integrals (9) simplify to

$$
\begin{aligned}
I_{j \mid i}^{(1)} & =\int_{0}^{l_{j \mid i}} d y_{1} \frac{e^{\beta U_{j \mid i}\left(y_{1}\right)}}{D_{j \mid i}\left(y_{1}\right)} \simeq \int_{x_{j \mid i}^{*}-\epsilon}^{x_{j \mid i}^{*}+\epsilon} d y_{1} \frac{e^{\beta U_{j \mid i}\left(y_{1}\right)}}{D_{j \mid i}\left(y_{1}\right)}, \\
I_{j \mid i}^{(2)} & =\int_{0}^{l_{j \mid i}} d y_{1} \int_{0}^{y_{1}} d y_{2} \frac{e^{\beta U_{j \mid i}\left(y_{1}\right)-\beta U_{j \mid i}\left(y_{2}\right)}}{D_{j \mid i}\left(y_{1}\right)} \\
& \simeq \int_{x_{j \mid i}^{*}-\epsilon}^{x_{j \mid i}^{*}+\epsilon} d y_{1} \int_{0}^{y_{1}} d y_{2} \frac{e^{\beta U_{j \mid i}\left(y_{1}\right)-\beta U_{j \mid i}\left(y_{2}\right)}}{D_{j \mid i}\left(y_{1}\right)} \\
& \simeq I_{j \mid i}^{(1)} \int_{0}^{x_{j \mid i}^{*}} d y_{2} e^{-\beta U_{j \mid i}\left(y_{2}\right)},
\end{aligned}
$$

where we assume $\epsilon \ll l_{j \mid i}$. The (saddle-point) approximations in the first and third lines of Eq. (D3) follow from $e^{\beta U_{j \mid i}}\left(y_{1}\right)$ being largest in the vicinity of $y_{1} \simeq x_{j \mid i}^{*}$ (see Fig. 5). The last approximation follows from $y_{2} \leq y_{1} \simeq x_{j \mid i}^{*}$ and hence, $\int_{0}^{y_{1}} d y_{2} e^{\beta U_{j \mid i}\left(y_{2}\right)} \simeq \int_{0}^{x_{j \mid i}^{*}} d y_{2} e^{-\beta U_{j \mid i}\left(y_{2}\right)}$. Inserting Eq. (D3) into the splitting probability in Eq. (10), and the mean exit time in Eq. (S50) in the Supplemental Material [75] yields the asymptotic rate of jumping from state $i$ to state $j$,

$$
\begin{aligned}
w_{i \rightarrow j} & =\phi_{j \mid i}^{\text {loc }} /\langle t\rangle_{i}^{\text {exit }} \simeq \frac{1}{I_{j \mid i}^{(1)}} \sum_{k \in \mathcal{N}_{i}^{*}} \int_{0}^{x_{k \mid i}^{*}} e^{-\beta U_{k \mid i}\left(y_{2}\right)} d y_{2}, \\
& \equiv \frac{e^{\beta \mathcal{U}_{i}-\beta \mathcal{F}_{i}}}{I_{j \mid i}^{(1)}},
\end{aligned}
$$

where in the last step we define the free energy of state $i$, $\mathcal{F}_{i}=-k_{B} T \ln \mathcal{Z}_{i}$ to be given by the partition function 
$\mathcal{Z}_{i}=e^{-\beta \mathcal{F}_{i}} \equiv \sum_{k \in \mathcal{N}_{i}} \int_{0}^{x_{k \mid i}^{*}} e^{-\beta \mathcal{U}_{i}-\beta U_{j \mid i}(x)} d x$. Note that $\mathcal{U}_{i}$ denotes the energy at node $i$ since $U_{j \mid i}(0)=0$. Inserting the rates Eq. (D4) along with Eq. (17) into the left-hand side of Eq. (20) yields the right-hand side of Eq. (20). This completes the proof of the local detailed balance relation in the limit of a timescale separation.

Let us now briefly comment on transition-path times in the limit of a timescale separation. Since, high free-energy barriers between any pair of state will eventually render all higher-order integrals $I_{i \mid j}^{(k)}$ negligibly small if $k \geq 3$, the transition-path time $\langle\delta t\rangle_{j \mid i}^{\mathrm{tr}}=I_{j \mid i}^{(3)} / I_{j \mid i}^{(1)}$ is negligibly short compared to the mean exit time from state $i$. More precisely, it has been found for a parabolic barrier that the transition-path time scales $\propto\left|U_{j \mid i}{ }^{\prime \prime}\left(x_{j \mid i}^{*}\right)\right|^{-1} \ln B_{j \mid i}$ $[24,30,70,116,117]$, i.e., decreases with $B_{j \mid i}$ due to $\left|U_{j \mid i}{ }^{\prime \prime}\left(x_{j \mid i}^{*}\right)\right|^{-1} \propto 1 / B_{j \mid i}[70]$, while the exit time grows much faster $[66,83,118]$, i.e., $\propto e^{\beta B_{j \mid i}}$. One can show that a rectangular-shaped potential with a barrier height $B_{j \mid i}$ in fact yields a constant finite transition-path time $\langle\delta t\rangle_{j \mid i}^{\mathrm{tr}}=$ $I_{j \mid i}^{(3)} / I_{j \mid i}^{(1)}$ in the limit $B_{j \mid i} \rightarrow \infty$, while at the same time the exit time diverges $\propto e^{\beta B_{j \mid i}} \rightarrow \infty$. The shape of the barrier may therefore decide whether or not the transition-path time is affected by the barrier height [70].

\section{Generalization to discontinuous local potentials and discontinuous diffusion landscapes}

We first explain how one deals with discontinuous local potentials in general. Next, we account for possible discontinuities in the diffusion landscape by removing them through a linear stretch of coordinates. Therefore, discontinuous diffusion landscapes can always be accounted for by mapping the coordinate system onto a continuous diffusion landscape but with possible discontinuities in the local potential.

\section{a. Discontinuous local potential}

Let us begin with a discontinuous "diverging force kick" at the node $i$ toward state $j$ which effectively means $F_{j \mid i}(x)=F_{j \mid i}^{\text {cont }}(x)+\Delta U_{j \mid i} \delta(x)$, where $F_{j \mid i}^{\text {cont }}(x)$ is some continuous force field, $\delta(x)$ denotes the Dirac delta function, and $\Delta U_{j \mid i}$ is the strength of the discontinuity. The "force kick" yields the potential $U_{j \mid i}(x)=-\Delta U_{j \mid i}-$ $\int_{0}^{x} F_{j \mid i}^{\text {cont }}\left(x^{\prime}\right) d x^{\prime}$. The local potential has a discontinuity once $U_{j \mid i}(0)=-\Delta U_{j \mid i} \neq 0$. A single discontinuity between states $i$ and $j$ is schematically depicted in Fig. 13 (see blue line). The transition-path time is not affected by such "kicks" since the transition path spans the time interval after the last passage of state $i$ until the first entrance into state $j$, which can be confirmed by the following argument. To formally avoid a discontinuity, we replace the

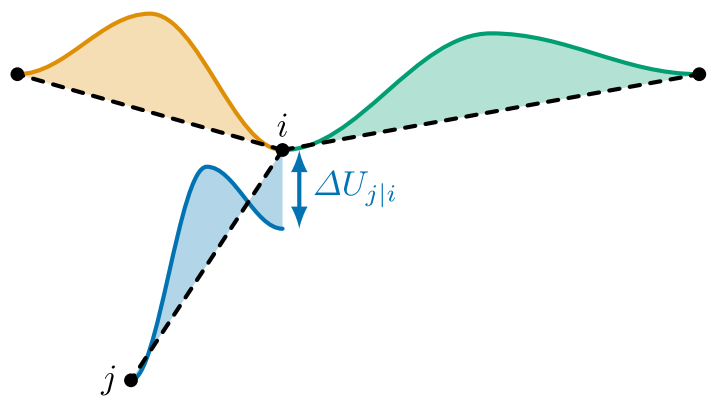

FIG. 13. Discontinuous potential. Local potential between state $i$ three neighbor states. Along the leg from state $i$ to state $j$ the potential has a discontinuity of strength $\Delta U_{j \mid i}$.

discontinuity $\Delta U_{j \mid i} \delta(x)$ by a smoothened force $\Delta U_{j \mid i} / \epsilon$ within the interval $0 \leq x \leq \epsilon$ and afterward take the limit $\epsilon \rightarrow 0$. The auxiliary integrals according to Eq. (9) become $\lim _{\epsilon \rightarrow 0} I_{j \mid i}^{(2 k-1)}=\left[\left.I_{j \mid i}^{(2 k-1)}\right|_{\Delta U_{j \mid i}=0}\right] \times e^{-\beta \Delta U_{j \mid i}} \quad$ and $\lim _{\epsilon \rightarrow 0} I_{j \mid i}^{(2 k)}=\left.I_{j \mid i}^{(2 k)}\right|_{\Delta U_{j \mid i}=0} \quad($ for $k=1,2 \ldots)$. Since all the odd-valued $k$ auxiliary integrals are affected by the discontinuity in precisely the same manner “ $I_{j \mid i}^{(2 k-1)} \propto e^{-\beta \Delta U_{j \mid i}}$," we find that the first two moments of transition-path time Eq. (11) are not affected by the discontinuity.

Importantly, a kick of strength $\Delta U_{j \mid i}$ affects the splitting probability $\phi_{j \mid i}^{\text {loc }}$ of choosing a transition due to $\phi_{j \mid i}^{\text {loc }} \propto$ $1 / I_{j \mid i}^{(1)} \propto e^{\beta \Delta U_{j \mid i}}[$ cf. Eqs. (9) and (10)]. Since the dwell time is affected by both the splitting probability and the transition-path time [cf. Eqs. (9) and (12)], a force kick of strength $\Delta U_{j \mid i}$ does affect the dwell-time statistics. Therefore, as an interim summary, we find that force kicks arising from a discontinuous local potential (see Fig. 13) affect both the splitting probability and the dwell-time statistics, whereas the transition-path time is not affected.

\section{b. Discontinuous diffusion landscape}

Discontinuous diffusion landscapes, i.e., $D_{j \mid i}(x)$ satisfying $D_{j \mid i}(0) \neq D_{k \mid i}$ for some $k \neq i$, are dealt with in the following manner. First, we locally rescale the coordinate system such that the discontinuity disappears. Specifically, we locally stretch the coordinates between nodes $i$ and $j$, $l_{j \mid i}$, homogeneously by a factor $\alpha_{j \mid i}\left(l_{j \mid i} \rightarrow \alpha_{j \mid i} l_{j \mid i}\right)$ to obtain a rescaled diffusion landscape $\alpha_{j \mid i}^{2} D_{j \mid i}\left(x / \alpha_{j \mid i}\right)$ and a correspondingly rescaled local potential $U_{j \mid i}\left(x / \alpha_{j \mid i}\right)-$ $\beta^{-1} \ln \alpha_{j \mid i}$, where $\beta^{-1}=k_{B} T$ is the thermal energy (see also Sec. II.E in Ref. [48]). By choosing $\alpha_{j \mid i}$ such that the diffusion landscape becomes continuous, we obtain a mapping from a discontinuous diffusion landscape onto a continuous one. Hence, discontinuous diffusion landscapes can be removed entirely via a linear change of local coordinates. Such a rescaling gives rise to a discontinuous 
potential - a problem we have already solved above. In this sense, all of the results presented here apply to dynamics on a graph with both discontinuous diffusion and discontinuous local potentials equally well. Notably, the results derived in the Supplemental Material Sec. III [75], i.e., Eqs. (10)-(12) in Sec. II D, can be used unaltered in the case of discontinuities in the potential and diffusion landscapes.

\section{APPENDIX E: MODEL PARAMETERS AND ADDITIONAL CONFIRMATION OF THE MAIN PRACTICAL RESULT}

In Appendix E 1, we provide details about the "synthetic" model discussed in Fig. 3 and Appendix C 3. We then define the catch-bond model in Appendix E2, which is discussed in Sec. V B [see Figs. 1(d), 7(b), and 7(c)]. The model from Figs. 7(d)-7(f) is provided in Appendix E3, where we also derive the upper bound depicted in Fig. 7(f). The lower bound in Fig. 7(f) is proven in Appendix E 4 and shown to saturate in Appendix E 5. In addition, we further corroborate all of our main findings. In particular, we verify symmetry (i) in Eq. (3) in Fig. 19(c). We test the reflection symmetry of the transition-path time [symmetry (ii) in Eq. (3)] in Fig. 21 as well as Table VII. In Tables IV and VI and Fig. 17, we corroborate our main practical result shown in Sec. II D.

\section{Dynamics in the synthetic network from Fig. 3 and Appendix C 3}

We briefly state all model parameters and then provide details about the analysis. Moreover, we use the model to corroborate the main practical result (see Sec. II D).

\section{a. Definition of the dynamics}

The synthetic network in Fig. 3(a) [see also Fig. 14(a)] is chosen to have one "slow" link between states 1 and 3

TABLE IV. Mean versus asymptotics. Each "experimental" value is deduced from $N_{\text {sim }}=4 \times 10^{5}$ simulated exits from each state generated by the stochastic Milstein scheme with $\Delta t=10^{-4}$. The "theory" values are obtained from a numerical evaluation of the results in Sec. IID [see also Eqs. (S47) and (S50) in the Supplemental Material [75]]. Each experimental value has a relative statistical error of about $1 / \sqrt{N_{\text {sim }}} \approx 0.0016$.

\begin{tabular}{lccccc}
\hline \hline & \multicolumn{2}{c}{ Mean exit rate $1 /\langle t\rangle_{i}^{\text {exit }}$} & & \multicolumn{2}{c}{ Rate $\mu_{i}=2\langle t\rangle_{i}^{\text {exit }} /\left\langle t^{2}\right\rangle_{i}^{\text {exit }}$} \\
\cline { 2 - 3 } State $i$ & Experiment & Theory & & Experiment & Theory \\
\hline 1 & 0.0617 & 0.0618 & & 0.0634 & 0.0636 \\
2 & 0.7496 & 0.7523 & & 0.7909 & 0.7934 \\
3 & 0.1140 & 0.1143 & & 0.0960 & 0.0961 \\
4 & 0.7509 & 0.7523 & & 0.7929 & 0.7934 \\
5 & 0.7523 & 0.7523 & & 0.7944 & 0.7934 \\
\hline \hline
\end{tabular}

being separated by a connection of length $l_{1 \mid 3}=l_{3 \mid 1}=$ 12.5518 with embedded diffusion coefficient $D_{3 \mid 1}=$ $D_{1 \mid 3}=1+4 \times 16\left(x / l_{3 \mid 1}-1\right)^{2}\left(x / l_{3 \mid 1}\right)^{2}$ and local force $F_{1 \mid 3}(x)=32 k_{B} T\left(2 x / l_{1 \mid 3}-1\right)\left(1-x / l_{1 \mid 3}\right) x / l_{1 \mid 3}^{2} \quad$ (corresponding to a local potential $\beta U_{1 \mid 3}=\beta U_{3 \mid 1}=16\left(x / l_{3 \mid 1}-\right.$ $1)^{2}\left(x / l_{3 \mid 1}\right)^{2}$ with a $1 k_{B} T$ barrier). Note that the local force is illustrated in Fig. 4(a), and the corresponding local potential is taken from Eq. (5). To assure the mildest of conditions, all remaining states are chosen to be separated by the same distance $l_{j \mid i}=1$, diffusion landscape $D_{j \mid i}=1$, and have the same force field $F_{j \mid i}(x)=96 k_{B} T \times(2 x /$ $\left.l_{j \mid i}-1\right)\left(1-x / l_{j \mid i}\right) x / l_{j \mid i}^{2}$ [i.e., local potential $\beta U_{j \mid i}(x)=$ $48(1-x)^{2} x^{2}$, which corresponds to a $3 k_{B} T$ barrier separating each pair of states]. This network (globally) satisfies detailed balance, since $U_{j \mid i}\left(l_{j \mid i}\right)=\mathcal{U}_{j}-\mathcal{U}_{i}$ for all $i, j$ with $\mathcal{U}_{i}=\mathcal{U}_{j}=0$ [for the definition of detailed balance, see paragraph including Eq. (5) or Appendix D 1].

\section{b. Simulation of microstate dynamics}

We generate individual trajectories using the stochastic Milstein scheme from Appendix A 3 with time increment $\Delta t=10^{-4}$. A short segment of the trajectory is shown in Fig. 3(a). In total, we simulate 400000 exits from each state and evaluate the probability density of the waiting time between all pairs of states. The results are summarized Figs. 14(b)-14(f), while Figs. 14(g)-14(i) display the same probability densities on a semilogarithmic scale. The probability densities depicted in Fig. 3(c) in the main text are taken from Fig. 14(d), while the inset in Fig. 3(c) represents Fig. 14(i). The gray lines in Fig. 14 corresponding to the solid black line in Fig. 3(c) denote the long-time asymptotics of the waiting-time distribution, which are determined as explained in the following.

\section{c. Analysis of the long-time asymptotics}

The long-time asymptotics of waiting-time density in state $i$ becomes a single exponential decay $\psi_{j \mid i} \propto e^{\mu_{i}^{\infty} t}$ with the same exponent $\mu_{i}^{\infty}$ for all exits to states $j$ adjacent to $i$. One can show that this implies the long-time asymptotics to be determined by $\mu_{i}^{\infty}=\lim _{n \rightarrow \infty} n\left\langle t^{n-1}\right\rangle^{\text {exit }} /\left\langle t^{n}\right\rangle^{\text {exit }}$. Note that when the waiting-time distribution and long-time asymptotics coincide $[66,83,118]$, one can instead simply use $(n=1)$, i.e., $\mu_{i}^{\infty} \approx 1 /\langle t\rangle^{\text {exit }}$. For examples violating the latter assumption, it turns out that taking $n=2$ provides a fairly good estimate $\mu_{i}=2\langle t\rangle^{\text {exit }} /\left\langle t^{2}\right\rangle^{\text {exit }} \approx \mu_{i}^{\infty}$ (see also Ref. [110]). We deduce the mean exit time and $\mu_{i}$ both from the theory (Sec. II D) and the simulation. These estimates are shown in Table IV. The thick gray lines in Fig. 14 are deduced from the theory values in Table IV. This corroborates the results in Sec. II D and shows that the long-time asymptotics can fairly accurately be determined from the first two moments of the exit time. 

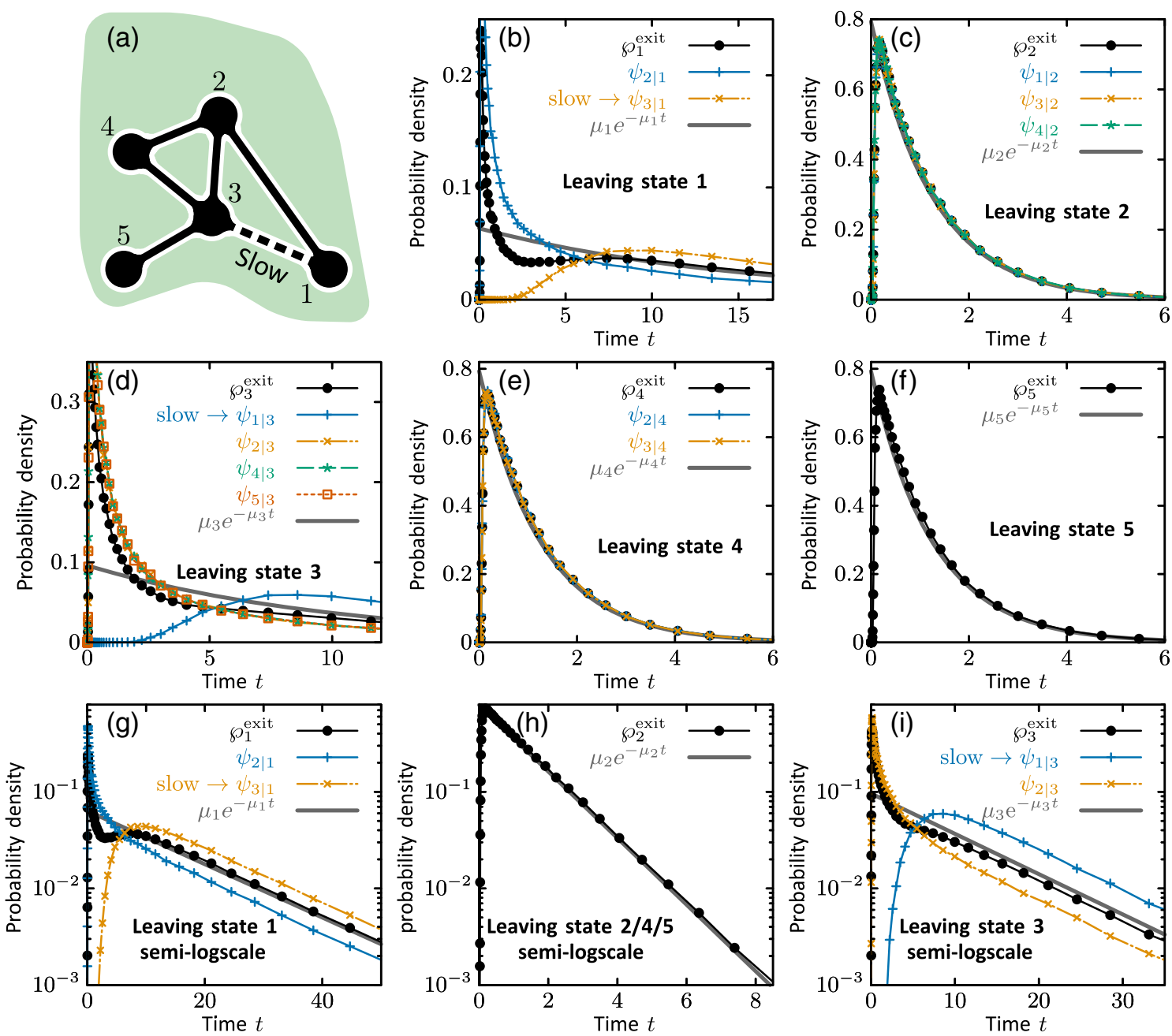

FIG. 14. State-to-state kinetics in the synthetic network. (a) Schematics of the network with a slow transition between states 1 and 3 (dashed line). All remaining connections (solid lines) are chosen to be equally fast with $3 k_{B} T$ barriers separating the network states. (b)-(f) Probability density of local residence time $\wp_{j \mid i}^{\text {loc }}$ starting from (b) $i=1$, (c) $i=2$, (d) $i=3$, (e) $i=4$, and (f) $i=5$, which are deduced from 400000 repeated exit events, respectively. The gray solid line denotes the estimated long-time asymptotics with the values from Table IV. The densities of leaving states 2, 4, and 5 are identical for the synthetic model. (g)-(i) Probability densities on a log scale; redundant densities are omitted since $\wp_{2}^{\text {exit }}=\wp_{4}^{\text {exit }}=\wp_{5}^{\text {exit }}$ holds, and all histograms plotted in (c) and (e) correspond to the same density, respectively, as well as $\wp_{2 \mid 3}^{\text {loc }}=\wp_{4 \mid 3}^{\text {loc }}=\wp_{5 \mid 3}^{\text {loc }}$.

\section{d. Slow transitions amplify the long-time asymptotics}

Whenever transitions are slow, we observe in Fig. 14 that the long-time asymptotics of the local probability density lies above the normalized gray line, which can be explained as follows. When transitions are long, the probability density $\psi_{j \mid i}(t)$ becomes negligibly small on timescales shorter than the transition time $t \lesssim\langle\delta t\rangle_{j \mid i}^{\mathrm{tr}}$. Since $\psi_{j \mid i}(t)$ must be normalized $\int_{0}^{\infty} \psi_{j \mid i}(t) d t=1$, one inevitably requires more weight of the probability density at long times. Note that all lines plotted in Figs. 14(b)-14(i) are probability densities which are normalized to unity. In other words, the blue solid line in Fig. 14(i) is above the gray thick line at long times since it is below the thick gray line at short times.

\section{Catch-bond analysis}

In this subsection, we provide details about the catch-bond analysis shown in Figs. 1(d), 7(b), and 7(c). Dissecting the lifetime of a bond into the dwell and transition time, we also corroborate symmetry (i) entering the main result in Eq. (3) [see Fig. 19(c)].

\section{a. The model}

We employ a so-called switch catch-bond model [67] with parameters chosen to reproduce experimental results on bacterial adhesion bonds [34,35] (see also Ref. [36] for related experiments). The local potential, Eq. (5), along the $j$ th pathway $(j=1,2)$ is decomposed into $U_{j \mid 0}(x)=$ $U_{j \mid 0}^{(0)}(x)+U^{\text {load }}(F, x)$, where $U_{j \mid 0}^{(0)}(x) \equiv U^{\text {load }}(0, x)$ is the 
(free-) energy profile at zero pulling force, and $U^{\text {load }}(F, x)$ accounts for a nonzero pulling force $F$. The potential along pathway $1 U_{1 \mid 0}(x)$ and along pathway $2 U_{2 \mid 0}(x)$ is depicted in Fig. 15, where solid lines represent potential values at zero pulling force $F=0$, dashed lines show the tilted potential under a moderate force $F=30 \mathrm{pN}$, and the dotted line corresponds to a pulling force $F=60 \mathrm{pN}$. Blue lines depict the potential along the fast pathway 1 , and orange lines the potential along the slow pathway 2 . The potential is formally defined as follows. Defining the scaled dimensionless distance $\tilde{x} \equiv x /(4.14 \mathrm{~nm})$, the potentials are given by

$$
\begin{aligned}
\beta U^{\text {pull }}(x, F) & =\frac{F}{1 \mathrm{pN}} \times \begin{cases}50 \tilde{x}^{2} & \text { if } \tilde{x} \leq 0.01, \\
\tilde{x}-0.005 & \text { if } \tilde{x}>0.01,\end{cases} \\
\beta U_{1 \mid 0}^{(0)}(x) & = \begin{cases}8500 \tilde{x}^{2} & \text { if } \tilde{x} \leq 0.01714, \\
5.1-8160(\tilde{x}-0.035)^{2} & \text { if } 0.01714<\tilde{x} \leq 0.06=l_{1 \mid 0} /(4.14 \mathrm{~nm}),\end{cases} \\
\beta U_{2 \mid 0}^{(0)}(x) & = \begin{cases}258.228 \tilde{x}^{2} & \text { if } \tilde{x} \leq 0.16458, \\
8.5-1200(\tilde{x}-0.2)^{2} & \text { if } 0.16458<\tilde{x} \leq 0.28417, \\
-1.6+6378.95(\tilde{x}-0.3)^{2} & \text { if } 0.28417<\tilde{x} \leq 0.33553, \\
12-9264.39(\tilde{x}-0.36)^{2} & \text { if } 0.33553<\tilde{x} \leq 0.397=l_{2 \mid 0} /(4.14 \mathrm{~nm}),\end{cases}
\end{aligned}
$$

where $l_{1 \mid 0}=0.06 \times 4.14 \mathrm{~nm}=0.248 \mathrm{~nm}$ and $l_{2 \mid 0}=0.397 \times$ $4.14 \mathrm{~nm}=1.64 \mathrm{~nm}$. The dimensionless unit length $\tilde{x}=$ $x /(4.14 \mathrm{~nm})$ is used to connect thermal energy and force according to $k_{B} T /(1 \mathrm{pN})=4.14 \mathrm{~nm}$. The diffusion coefficient is set to be constant $D_{j \mid 0}(x)=(4.14)^{2} \mathrm{~nm}^{2} \mathrm{~s}^{-1}=$ $17.1 \mathrm{~nm}^{2} \mathrm{~s}^{-1}$ along both pathways $j=1$ and $j=2$.

\section{b. Simulation results in Fig. 1(d)}

We propagate the microstate with the stochastic Runge-Kutta scheme given in Appendix A 4 with time increment $\Delta t=10^{-6} \mathrm{~s}$. Setting the initial distance to $x_{0}=0$, we simulate $10^{4}$ ruptures for each force

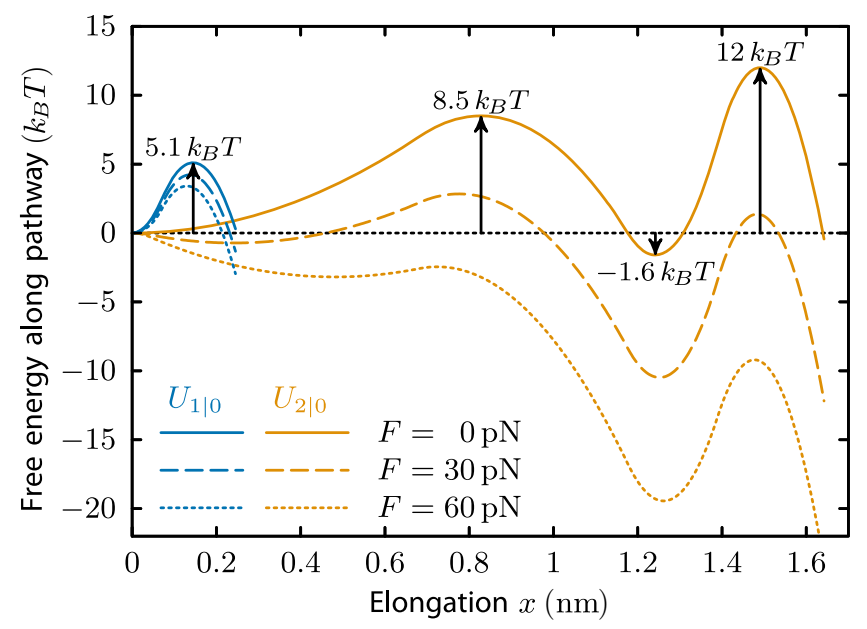

FIG. 15. Catch-bond free-energy landscape. The local potential is set to $U_{j \mid 0}(x)=U_{j \mid 0}^{(0)}(x)+U^{\text {load }}(F, x)$. The precise form of $U_{1 \mid 0}$ and $U_{2 \mid 0}$ is given in Eq. (E1). For a biophysical setting underpinned by the potential, see Fig. 5(a) in Ref. [35] [see also Fig. 1(d) here].
$F=0,5 \mathrm{pN}, \ldots, 85 \mathrm{pN}$, respectively. Some selected probability densities with a logarithmic (increasing) binning are shown in Fig. 16. Figure 16(a) depicts the probability densities on a semilogarithmic scale only over a short period of time $(4 \mathrm{~s})$. Figure 16 depicts the full-time range on a double-logarithmic scale, which after normalization of time $t \rightarrow t /\langle t\rangle^{\text {exit }}$, Fig. 16(c) allows us to conveniently depict the shape of all distributions simultaneously on a linear scale as in Fig. 16(d). That is, all scaled densities in Fig. 16(d) have the same scaled mean at $t /\langle t\rangle^{\text {exit }}=1$. We adopt the density belonging to rectangles $(F=20 \mathrm{pN})$ in Fig. 16 in the blue shaded plot in Fig. 1(d). In Table V, we list the mean rupture times along pathways 1 and 2, respectively, whereby the length of the orange and black bars in Fig. 1(d) along the $j$ th pathway represent the values $\langle t\rangle_{j \mid 0}$ at $F=20 \mathrm{pN}$ from the table.

In contrast to the experiment [34], we assume here that all trajectories instead of $99.2 \%$ start from $x=0$. We note that the fit of the experimental data carried out in Ref. [34] found the initial binding to take place with $99.2 \%$ in what was called state 1 , which corresponds here to the distance $x=0$. Correspondingly, about $0.8 \%$ of experimental ruptures carried out in Ref. [34] are estimated to start in the first intermediate minima along the slow path 2 (potential is depicted in Fig. 15 in the main text).

\section{c. Simulation of Figs. 7(b) and 7(c)}

Using all $10^{4}$ rupture events, we deduce in Fig. 17(a) (see symbols) the splitting probability $\phi_{2 \mid 0}=1-\phi_{1 \mid 0}$, the mean lifetime $\langle t\rangle^{\text {exit }}$, and its standard deviation $\sigma_{\text {exit }}=$ $\sqrt{\left\langle t^{2}\right\rangle^{\text {exit }}-\left(\langle t\rangle^{\text {exit }}\right)^{2}}$. The error bars denote the root-meansquare error. The theoretical lines in Fig. 17(a) are obtained by numerical integration of Eq. (9) along both pathways 

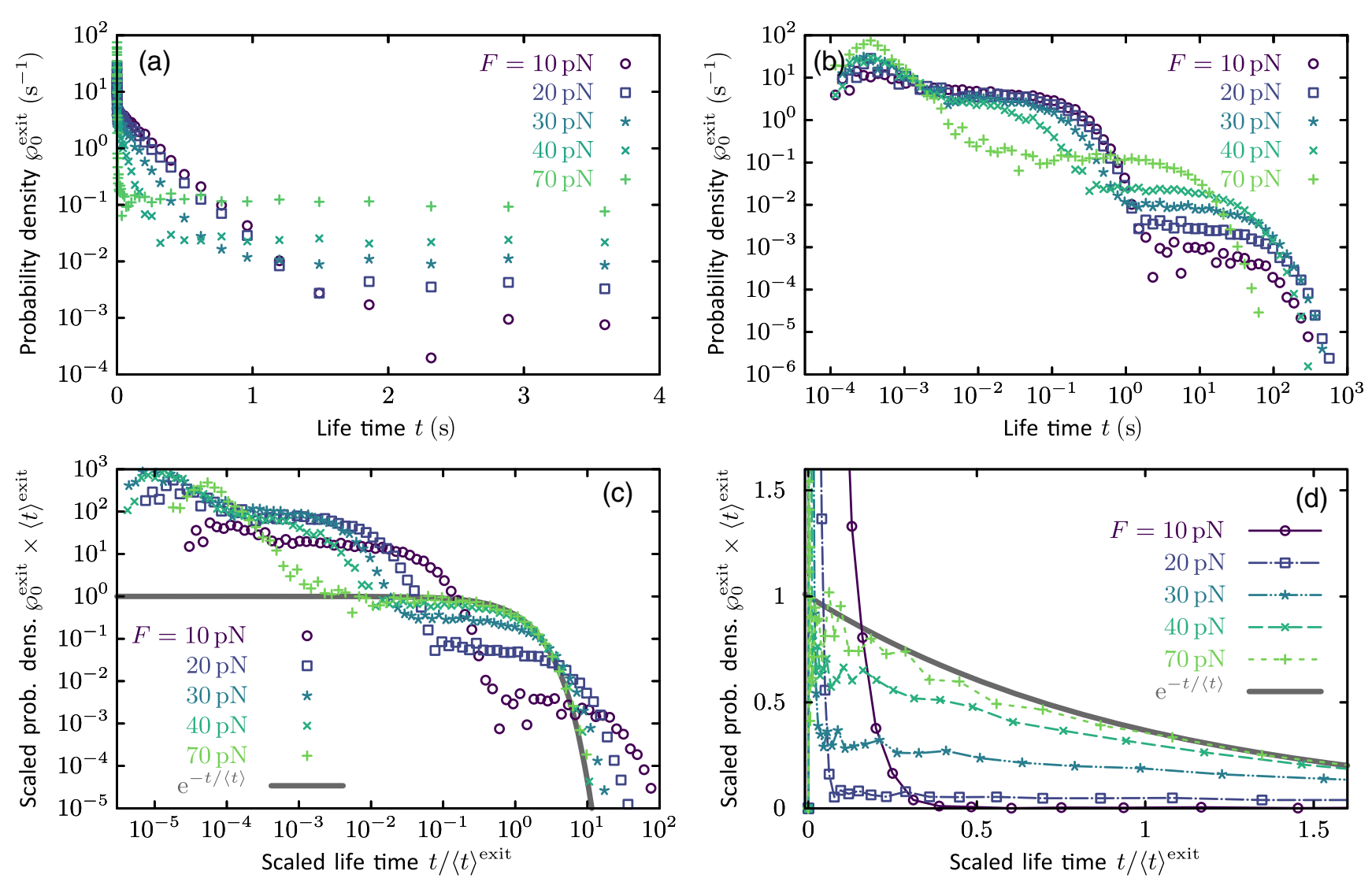

FIG. 16. Probability density of bond lifetime. (a) Probability densities on a semilogarithmic scale. (b) Probability densities on a log-log scale. (c) Scaled probability density and scaled time on a log-log scale; the mean lifetime corresponds to $t /\langle t\rangle$ exit $=1$. (d) Scaled probability densities on a linear scale. Each probability density is estimated from 10000 ruptures. All symbols are deduced from histograms with equidistant binning in logarithmic time.

(1 and 2) and consecutive use of Eqs. (10)-(13). This example nicely corroborates the validity of our results. In Figs. 7(b) and 7(c), the number of ruptures is chosen to be similar to those in typical experiments [34,36] (here 500 rupture events).

\section{d. Interplay of fast and slow transitions at finite statistics}

The interplay between fast and slow transitions can lead to a severe misinterpretation of experimental error estimates

TABLE V. Asymmetry of the mean rupture time. The mean rupture time $\langle t\rangle_{1 \mid 0}$ and $\langle t\rangle_{2 \mid 0}$ along pathways 1 and 2, respectively. We highlight the results with a strong asymmetry $\langle t\rangle_{2 \mid 0} /\langle t\rangle_{1 \mid 0}>100$.

\begin{tabular}{lccc}
\hline \hline Pulling force $F$ & $\phi_{1 \mid 0}$ & $\langle t\rangle_{1 \mid 0}$ & $\langle t\rangle_{2 \mid 0}$ \\
\hline $10 \mathrm{pN}$ & 0.98 & $2.81 \mathrm{~s}$ & $62 \mathrm{~s}$ \\
$20 \mathrm{pN}$ & 0.81 & $6.32 \mathrm{~s}$ & $77 \mathrm{~s}$ \\
$30 \mathrm{pN}$ & 0.44 & $2.51 \mathrm{~s}$ & $58 \mathrm{~s}$ \\
$40 \mathrm{pN}$ & 0.17 & $0.43 \mathrm{~s}$ & $33.2 \mathrm{~s}$ \\
$50 \mathrm{pN}$ & 0.07 & $0.015 \mathrm{~s}$ & $19.4 \mathrm{~s}$ \\
$60 \mathrm{pN}$ & 0.05 & $0.004 \mathrm{~s}$ & $11.35 \mathrm{~s}$ \\
\hline \hline
\end{tabular}

(here at low pulling force). To see this, we presume that we have only 200 ruptures measured [see Fig. 17(b)]. Clearly, errors are expected to become larger, whereas at low pulling forces we mistakenly estimate the errors to be far too small. To understand this, we need to inspect the full probability densities depicted in Fig. 16 (see circles, $F=10 \mathrm{pN}$ ). The probability density is negligibly small at values $t \leq\langle t\rangle^{\text {exit }} / 2$; i.e., the mean is mainly dominated by extremely rare and extremely long transitions. This situation becomes more severe at smaller forces. For example, at $F=5 \mathrm{pN}$ we do not encounter a single rupture along the slow path in the first 200 ruptures, which is why we experimentally would not be able to see them. This is the reason why the theory lines in Figs. 17(b) are 10 standard deviations away from the theory line at $F=5 \mathrm{pN}$. In other words, in reality, 200 ruptures alone at $F=5 \mathrm{pN}$ lead to the same quality of statistics as an experiment with only one or two ruptures.

\section{e. Detection of parallel transition paths according to Ref. [27]}

If we are able transition path times $\delta t$ directly, one can also evaluate the coefficient of variation $\sigma_{\text {tr }}^{2} /\langle\delta t\rangle^{2}=$ $\left(\left\langle\delta t^{2}\right\rangle-\langle\delta t\rangle^{2}\right) /\langle\delta t\rangle^{2}$. The result is shown in Fig. 18 . 
(a) 10000 ruptures

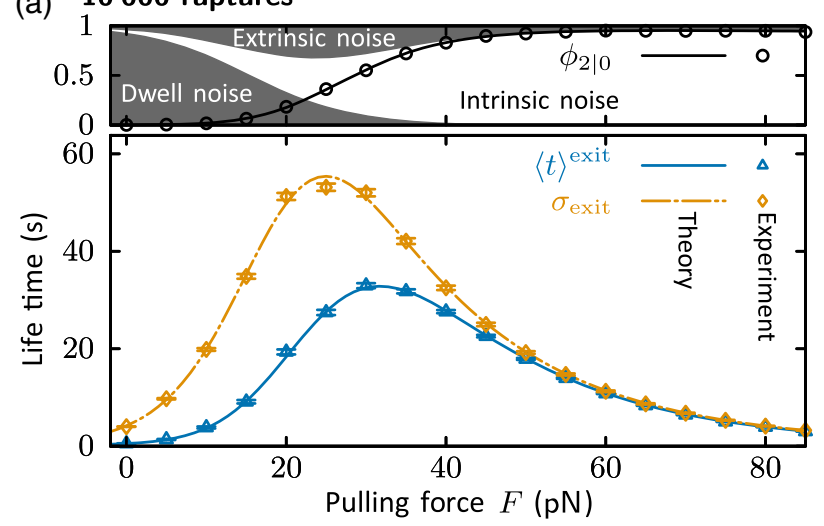

(b) 200 ruptures

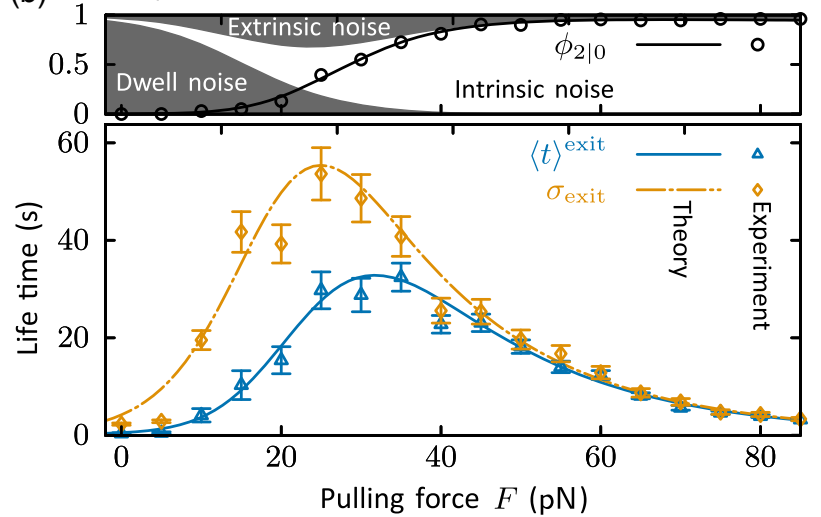

FIG. 17. Catch-bond analysis with improved statistics. The results for (a) $10^{4}$ ruptures and (b) 200 ruptures. The error bars denote the standard deviation and the lines the theoretical results.

The coefficient clearly exceeds 1 , which according to Ref. [27] correctly implies multidimensional (parallel) transition paths. Note in Sec. V B, we detect parallel transition paths "merely" from measuring the lifetime of the bond $t=\delta t+\tau$, which formally represents a firstpassage time.

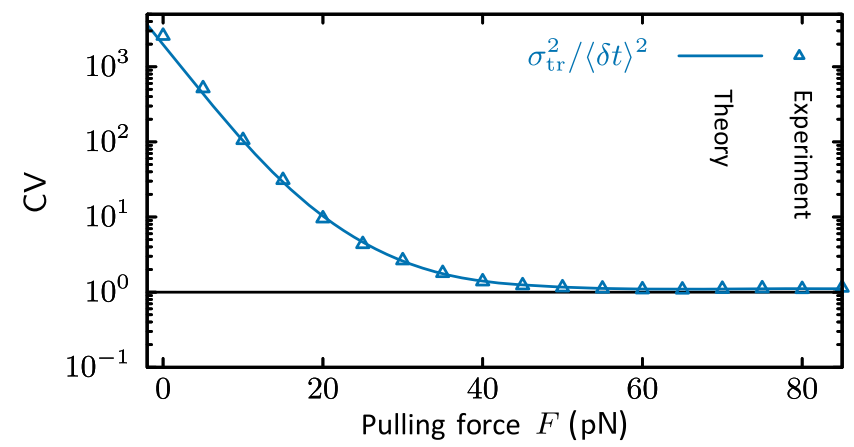

FIG. 18. Coefficient of variation of transition-path time. Symbols are obtained from $10^{4}$ ruptures as in Fig. 17, and the theory lines are deduced from Eq. (11). We define the mean transition time $\langle\delta t\rangle=\phi_{1 \mid 0}\langle\delta t\rangle_{1 \mid 0}+\phi_{2 \mid 0}\langle\delta t\rangle_{2 \mid 0}$ and variance $\sigma_{\text {tr }}^{2}=\phi_{1 \mid 0}\left\langle\delta t^{2}\right\rangle_{1 \mid 0}+$ $\phi_{2 \mid 0}\left\langle\delta t^{2}\right\rangle_{2 \mid 0}-\langle\delta t\rangle_{0}^{2}$.

\section{f. Dwell time is a property of the state}

Using the individual trajectories for the catch-bond system, we dissect the lifetime of a bond into a transition and dwell period according to Eq. (3) in the main text. For brevity, we merely show the result for $F=30 \mathrm{pN}$ at which the two paths are taken with approximately equal probability. In Fig. 19(a), we depict the histogram of the lifetime of the bond $\wp_{0}^{\text {exit }}(t)$ (see green stars), which is dissected into the statistics of dwelltime $\wp_{0}^{\mathrm{dwell}}$ (see blue circles) and the transition-time statistics $\wp_{0}^{\mathrm{tr}} \equiv \phi_{1 \mid 0} \wp_{1 \mid 0}^{\mathrm{tr}}+\phi_{2 \mid 0} \wp_{2 \mid 0}^{\mathrm{tr}}$ (see orange rectangles), respectively. The arrow "path 1" in Fig. 19(a) indicates $\phi_{1 \mid 0} \wp_{1 \mid 0}^{\mathrm{tr}}$, whereas the arrow "path 2" indicates $\phi_{2 \mid 0} \wp_{2 \mid 0}^{\mathrm{tr}}$. Note that the lifetime $\wp_{0}^{\text {exit }}$ is equal to the convolution of dwell- and transition-time distributions $\wp_{0}^{\text {exit }}=\wp_{0}^{\mathrm{tr}} * \wp_{0}^{\mathrm{d} w e l l}$, which signifies their statistical independence. Figure 19(b) depicts the probability density of logarithmic time $\ln t$, which is $t \wp(t)$ since $\int_{-\infty}^{\infty} t \wp(t) d[\ln t]=1$.

We now use the data to verify symmetry (i) in our second main result Eq. (3), which states that the dwell-time statistics is identically distributed along both pathways 1 and 2. To test this, we compare the histogram of dwell time along path $1 \wp_{1 \mid 0}^{\mathrm{dwell}}$ and the histogram along path $2 \wp_{2 \mid 0}^{\mathrm{d} w e l l}$ with the estimated probability density along both pathways $\wp_{0}^{\text {dwell }}$ in Fig. 19(c), where the inset depicts the results on a linear scale. Figure 19(c) nicely illustrates their distribution to be equal $\wp_{0}^{\text {dwell }}=\wp_{1 \mid 0}^{\text {dwell }}=\wp_{2 \mid 0}^{\text {dwell }}$ (deviations are merely arising from finite statistics). This example illustrates that the dwell-time statistics does not depend on the pathway of the rupture (states 1 and 2) but only on the initial state 0 ; i.e., the dwell-time statistics solely depends on the initial state (not on the final one). This example corroborates Eq. (3) in the main text. Using fast three-color singlemolecule Foster resonance energy transfer, it is possible to detect similar parallel transition paths in the binding of disordered proteins [29].

\section{g. Alternative experiment from Ref. [36]}

Finally, we want to comment on the effect of changing the length scale. Suppose the length $x$ is stretched by a factor $\lambda$ such that $U_{j \mid i}(x) \rightarrow U_{j \mid i}(x / \lambda)$, i.e., $F_{j \mid i}(x) \rightarrow F_{j \mid i}(x / \lambda) / \lambda$, which implies that the loading force $F$ becomes equivalent to the loading force $F / \lambda$ after rescaling. To address a related experiment [36] displaying quite different timescales and length scales, we need to scale the length by a factor $\lambda(\lambda \approx 3)$ such that the maximum lifetime is found at $F \approx 10 \mathrm{pN}$ as reported in Ref. [36] instead of $F \approx 30 \mathrm{pN}$, which is shown here in Fig. 17 (see also Ref. [34]). Moreover, scaling the diffusion constant $D \rightarrow \alpha D$ corresponds to an accelerated time, which rescales the bond lifetime $\propto \alpha^{-1} \lambda^{-2}$. To shift the maximum lifetime from $\simeq 30 \mathrm{~s}$ (see Fig. 17) to $1.2 \mathrm{~s}=$ $30 \mathrm{~s} / 25$ from the experiment in Ref. [36], we, in addition to the scaled location of the maximum, scale the diffusion constant by $\alpha=25 \times \lambda^{-2} \approx 2.78$. With these scaled units we 

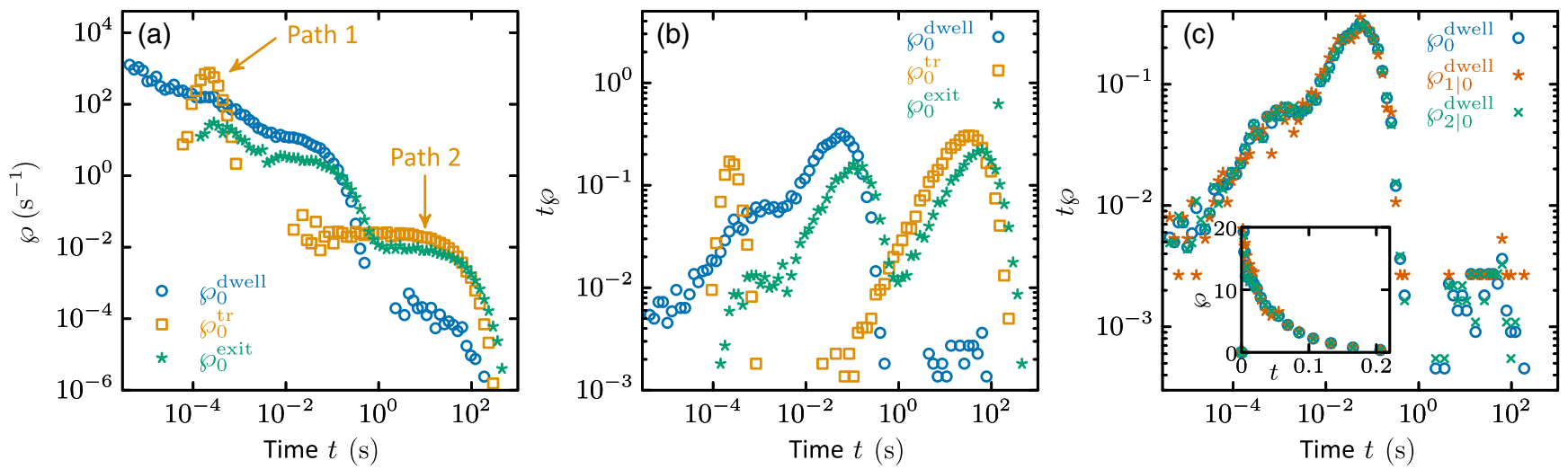

FIG. 19. Decomposition of the probability density of bond lifetime at pulling force $F=30 \mathrm{pN}$ into the probabilities of transition and dwell time, respectively. (a) Probability densities of bond lifetime $\wp^{\text {exit }}$, dwell time $\wp^{\text {dwell }}$, and transition time $\wp^{\text {tr }}$ on a log-log scale. (b) Result from (a) where the probability densities are transformed to logarithmic densities $t \wp(t)$, which is normalized according to $\int_{-\infty}^{\infty} t \wp(t) d(\ln t)=\int_{0}^{\infty} \wp(t) d t=1$. (c) Test of $\wp_{0}^{\mathrm{dwell}}=\wp_{1 \mid 0}^{\mathrm{dwell}}=\wp_{2 \mid 0}^{\mathrm{dwell}}$.

obtain the same plots as shown in Fig. 17 [see also Figs. 7(b) and 7(c) in the main text] but with the $x$ axis scaled by a factor of $1 / 3$, and the $y$ axis is scaled by a factor of $1 / 25$ to quantitatively account for different experiment reported in Ref. [36].

Summarizing, in this subsection we further confirm Eqs. (10)-(13) in the main text by numerical experiments, which are shown in Fig. 17 using more statistics (up to $10^{4}$ rupture events). We test the decomposition of the bond lifetime into its dwell and transition period according to Eq. (3) in the main text, and we corroborate the theoretical prediction that the dwell time indeed depends only on the initial state but not the final state [see Fig. 19(c)].

\section{ATPase with sine-wave potential}

\section{a. Model and energetics}

We assume the dynamics of an idealized ATPase to be described by the following model. The ATPase rotates stochastically about one axis and experiences an angledependent torque at rotation angle $\theta_{t}$ at time $t$. The torque is assumed to have the following two contributions:

(i) A rotational free-energy potential [see blue shaded lines Fig. 7(d)] that displays three well-defined rotational states (minima) that are separated by $120^{\circ}$. The free energy exerts a conservative torque proportional to the slope of the blue line. The potential is given by $U^{\operatorname{rot}}(\theta)=(B / 2)[1-\cos (\theta / 3)]$ with the implied conservative torque given by $-\partial_{\theta} U^{\mathrm{rot}}(\theta)$.

(ii) A nonequilibrium torque $M$ that embodies a sum of a mechanochemical force arising from the hydrolysis of an ATP molecule and a mechanical torque that is applied to the shaft. More precisely, a tight coupling with $M=\Delta \mu / 120^{\circ}-M^{\text {mech }}$ is assumed, where $\Delta \mu=\mu_{\mathrm{ATP}}-\mu_{\mathrm{ADP}}-\mu_{P_{i}}$ is the chemical free energy released in the hydrolysis reaction to adenosine diphosphate (ADP), ATP $\rightarrow$ ADP $+P_{i}$, and $M^{\text {mech }}$ reflects a mechanical torque [50].
In Figs. 7(d)-7(f), we set $B=5 k_{B} T$ and assume the diffusion coefficient to be constant and without loss of generality $D=1$. Moreover, we use scaled units $x_{t}=\theta_{t} / 120^{\circ}$; that is, distances are measured in units of a third of a revolution. Using the scaled coordinate, the local potential, which accounts for both torque (i) and mechanochemical force (ii), is given by $U_{ \pm}(x)=(B / 2)[1-\cos (2 \pi x)] \pm(M \times$ $\left.120^{\circ}\right) x$ with $l_{ \pm}=120^{\circ} / 120^{\circ}=1$, where " + " accounts for the potential along the counterclockwise direction and "-_" corresponds to the potential along the opposite direction. Detailed balance is established whenever the chemical free energy released per $120^{\circ}$ step is balanced by the mechanical torque (multiplied by $120^{\circ}$ ), i.e., $M=0$ [see item (ii) above].

For convenience, we restrict our analysis to a periodic rotation which has a sine-wave shape with barriers of height $B$ that separate two minima. Counting the minima in the counterclockwise direction yields the set of states $\Omega=\{1,2,3\}$, such that for each state $i \in \Omega$ the local potential formally reads $U_{i \pm 1 \mid i} \equiv U_{ \pm}$with the convention " $i-1=3$ if $i=1$ " and " $i+1=1$ if $i=3$." The sets of neighboring states are then $\mathcal{N}_{1}=\{2,3\}, \mathcal{N}_{2}=\{1,3\}$, and $\mathcal{N}_{4}=\{1,2\}$; that is, the three-state network is fully connected. We want to compare the minima-to-minima dynamics, which are generally non-Markovian, to a Markov kinetics corresponding to an exponentially distributed waiting time with the same expected time $\langle t\rangle^{\text {exit }}$ of leaving each minimum. Only two numbers become relevant, $B /\left(k_{B} T\right)$ and $M \times 120^{\circ} /\left(k_{B} T\right)$ representing, respectively, the barrier height separating two minima $B$ and the nonequilibrium driving $M$ in units of the thermal energy $k_{B} T$. The diffusion constant is set to $D_{ \pm}=1$. To obtain the numerical results in Figs. 7(e) and 7(f), we fix the barrier height to $B /\left(k_{B} T\right)=5$ and use the stochastic Runge-Kutta scheme (see Appendix A 4) with time increment $\Delta t=10^{-4}$ in dimensionless simulation units. We simulate all trajectories until we observe in total 500000 state-to-state changes (i.e., minima-to-minima transitions). Note that a different value for $D \neq 1$ would not affect Figs. 7(e) 


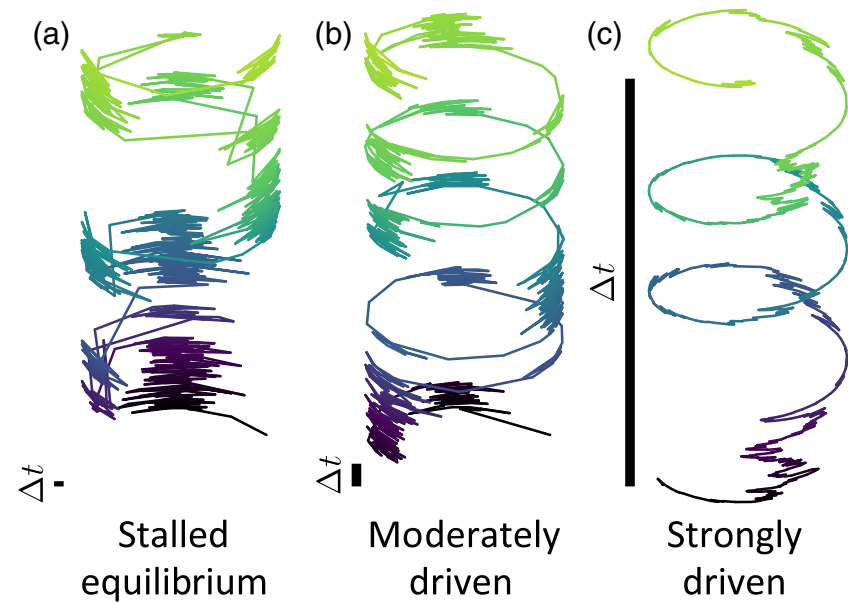

FIG. 20. Effect of nonequilibrium driving on single trajectories. Realization of a stochastic trajectory of the ATPase toy model as function of time (running from dark to bright) at (a) equilibrium $M=0$, (b) at moderate nonequilibrium driving $M \times 120^{\circ}=B=$ $5 k_{B} T$, and (c) at strong driving $M \times 120^{\circ}=20 k_{B} T$. The length of each trajectory is chosen to have the same average number of transitions. To compare the different time units, we add the bars $\Delta t$, which all span the same period of time.

and 7(f). The fluctuating rotational state as a function of time is illustrated in Fig. 20 for various strengths of driving $M \times 120^{\circ} /\left(k_{B} T\right)=0,5,20$, where each all-black bar represents an equal duration $\Delta t$, indicating that a stronger driving leads to faster rotation.

\section{b. Splitting probability}

The splitting probability involves the auxiliary integrals

$$
\begin{aligned}
& I_{+}^{(1)}=\int_{0}^{1} e^{\beta \frac{B}{2}[1-\cos (2 \pi x)]-\beta\left(M \times 120^{\circ}\right) x} d x, \\
& I_{-}^{(1)}=\int_{0}^{1} e^{\beta \frac{B}{2}[1-\cos (2 \pi x)]+\beta\left(M \times 120^{\circ}\right) x} d x,
\end{aligned}
$$

where we use Eq. (9) with $k=1$ and insert $U_{ \pm}(x)=$ $(B / 2)[1-\cos (2 \pi x)] \mp\left(M \times 120^{\circ}\right) x$ and $l_{ \pm}=1$. Using Eq. (10), one obtains after some algebra

$$
\phi_{+}=\frac{1 / I_{+}^{(1)}}{1 / I_{+}^{(1)}+1 / I_{-}^{(1)}}=\frac{e^{f}}{e^{f}+1}=1-\phi_{-},
$$

where we define $f=\beta\left(M \times 120^{\circ}\right)$. Note that splitting probabilities satisfy Eq. (2), which here corresponds to $\ln \left(\phi_{+} / \phi_{-}\right)=f$. Some values are listed in Table VI.

\section{c. Symmetry of the waiting-time distribution}

We prove in Appendix B a forward and backward symmetry of the transition time " $\wp_{+}^{\mathrm{tr}}(\delta t)=\wp_{-}^{\mathrm{tr}}(\delta t)$ " [i.e., symmetry (ii) in our second main result in Eq. (3)]. To numerically corroborate this main finding, we compare in Table VII the mean transition time along the forward + and backward - direction (as we explain in Appendix A 5). In Fig. 21, we further compare the entire probability densities. Because of the periodicity in each $120^{\circ}$ step and the forward or backward symmetry of the transition time $\wp_{+}^{\mathrm{tr}}(\delta t)=\wp_{-}^{\mathrm{tr}}(\delta t)$, Eq. (3) implies that the local waiting is given by $\wp_{ \pm}^{\text {loc }}(t)=\phi_{ \pm} \wp^{\text {exit }}(t)$, such that $\left\langle t^{k}\right\rangle_{ \pm}=\left\langle t^{k}\right\rangle^{\text {exit }}$. Therefore, the extrinsic transition noise vanishes, which in turn according to the proof in the last subsection in the Supplemental Material [75] implies the fluctuations to be sub-Markov: $\sigma_{\text {exit }}^{2} \equiv\left\langle t^{2}\right\rangle^{\text {exit }}-\left(\langle t\rangle^{\text {exit }}\right)^{2} \leq\left(\langle t\rangle^{\text {exit }}\right)^{2}$, that is, $\left\langle t^{2}\right\rangle^{\text {exit }} \leq 2\left(\langle t\rangle^{\text {exit }}\right)^{2}$.

A few comments are in order. The symmetry of the local mean waiting time $\langle t\rangle_{+}=\langle t\rangle_{-}$, was, to the best of our knowledge, first discovered in Ref. [64] for lattice models of kinesin motors (see also Ref. [65]). The extension to the entire distribution $\wp_{ \pm}^{\text {loc }}(t)=\phi_{ \pm} \wp^{\text {exit }}(t)$ was later found in studies for the stopping time of the thermodynamic entropy production in active molecular processes [59]. The symmetry allows us to simplify the discussion by merely focusing on the splitting probability $\phi_{ \pm}$and the exit-time distributions $\wp^{\text {exit }}(t)$.

TABLE VI. Comparing theory to simulation. Nonequilibrium driving is quantified in terms of $f \equiv M \times 120^{\circ} /\left(k_{B} T\right)$. The theoretical values for the splitting probability follow from Eq. (E3). By evaluating the auxiliary integrals in Eq. (9) and using Eqs. (S47) and (S50) in the Supplemental Material [75], we obtain the theoretical values for mean first exit time $\langle t\rangle^{\text {exit }}$ and the second moment of the exit time $\left\langle t^{2}\right\rangle^{\text {exit }}$, and therefrom the

\begin{tabular}{|c|c|c|c|c|c|c|}
\hline \multirow[b]{2}{*}{ Nonequilibrium driving $f$} & \multicolumn{2}{|c|}{ Splitting probability $\phi_{-}=1-\phi_{+}$} & \multicolumn{2}{|c|}{ Mean exit time $\langle t\rangle^{\text {exit }}$} & \multicolumn{2}{|c|}{ Standard deviation $\sigma_{\text {exit }}$} \\
\hline & Theory & Experiment & Theory & Experiment & Theory & Experiment \\
\hline 0 & 0.500000 & 0.500910 & 5.4115 & 5.4158 & 5.3561 & 5.3533 \\
\hline 2 & 0.119203 & 0.119490 & 3.7064 & 3.7066 & 3.6513 & 3.6469 \\
\hline 5 & 0.006692 & 0.006654 & 1.2488 & 1.2474 & 1.1951 & 1.1940 \\
\hline 10 & 0.000045 & 0.000048 & 0.2910 & 0.2916 & 0.2425 & 0.2428 \\
\hline 20 & $2.06 \times 10^{-9}$ & 0 & 0.0703 & 0.0705 & 0.0350 & 0.0350 \\
\hline
\end{tabular}
standard deviation $\sigma_{\text {exit }}=\sqrt{\left\langle t^{2}\right\rangle^{\text {exit }}-\left(\langle t\rangle^{\text {exit }}\right)^{2}}$. Note that for $f=20$ the system is driven so strongly that no backward transition is observed in 500000 trajectories, which is why we experimentally determine $\phi_{-}=0$. 
TABLE VII. Test of forward and backward symmetry of mean transition time. The transition time is evaluated from 500000 state-to-state changes. The statistical error in the mean transition time $\langle t\rangle_{-}$denotes the estimated approximately $95 \%$ confidence interval.

\begin{tabular}{lccc}
\hline \hline $\begin{array}{l}\text { Nonequilibrium } \\
\text { driving }\end{array}$ & \multicolumn{2}{c}{ Mean transition time } & $\begin{array}{c}\text { Number of } \\
\text { steps backward }\end{array}$ \\
\cline { 2 - 3 } 0 & $\langle\delta t\rangle_{+}^{\text {tr }}$ & $\langle\delta t\rangle_{-}^{\text {tr }}$ & 250457 \\
2 & 0.0656 & $0.0656 \pm 0.0001$ & 59745 \\
5 & 0.0659 & $0.0660 \pm 0.0002$ & 3327 \\
10 & 0.0676 & $0.0685 \pm 0.0009$ & 24 \\
\hline \hline
\end{tabular}

\section{d. Exit-time statistics and implied number of transitions}

The number of exits after time $t, n_{t}$, where one exit corresponds to the event of leaving one minima and reaching any other minima for the first time, is stochastic and influenced solely by the exit time $\wp^{\text {exit }}(t)$. As we explain above, the distribution of the waiting time is the same along both directions + and -, i.e., $\psi_{ \pm}=\wp_{ \pm}^{\text {loc }}(t) / \phi_{ \pm}^{\text {loc }}=\wp^{\text {exit }}(t)$. At long times, the central limit theorem for renewal processes [16] renders $n_{t}$ asymptotically normally distributed with mean $\left\langle n_{t}\right\rangle \simeq t /\langle t\rangle^{\text {exit }}$ and variance $\operatorname{var}\left(n_{t}\right) \equiv\left\langle n_{t}^{2}\right\rangle-$ $\left\langle n_{t}\right\rangle^{2} \simeq t \sigma_{\text {exit }}^{2} /\left(\langle t\rangle^{\text {exit }}\right)^{3}=t\left[\left\langle t^{2}\right\rangle^{\text {exit }}-\left(\langle t\rangle^{\text {exit }}\right)^{2}\right] /\left(\langle t\rangle^{\text {exit }}\right)^{3}$, where $\simeq$ denotes equality $=$ in the limit $t \rightarrow \infty$ (see also Ref. [37]). Applying the central limit theorem for the meansquare angular deviation $\left\langle\delta \theta_{t}^{2}\right\rangle \equiv\left\langle\left[\theta_{t}-\left\langle\theta_{t}\right\rangle\right]^{2}\right\rangle$, we obtain

$$
\begin{aligned}
\frac{\left\langle\delta \theta_{t}^{2}\right\rangle}{\left(120^{\circ}\right)^{2}} & =4 \phi_{+} \phi_{-}\left\langle n_{t}\right\rangle+\left(\phi_{+}-\phi_{-}\right) \operatorname{var}\left(n_{t}\right) \\
& \simeq 4 \phi_{+} \phi_{-} \frac{t}{\langle t\rangle^{\text {exit }}}+\left(\phi_{+}-\phi_{-}\right) \frac{t \sigma_{\text {exit }}^{2}}{\left(\langle t\rangle^{\text {exit }}\right)^{3}},
\end{aligned}
$$

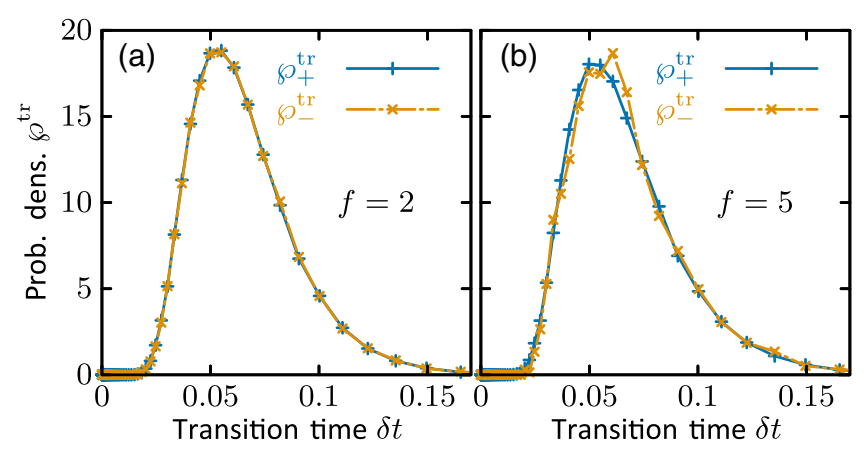

FIG. 21. Test of forward and backward symmetry of transition time. Probability density of transition time $\wp_{ \pm}^{\text {tr }}$ in forward + and backward - direction for weakly (a) $f=2$ and (b) $f=5$. The number of trajectories entering $\wp_{-}^{\text {tr }}$ are (a) $n_{-}=59745$ and (b) $n_{-}=3327$ (see also Table VII). Deviations between blue and orange lines in (b) are due to finite statistics $\left(n_{-}=3327\right)$; see also Table VII for the number of observed backward transitions. where in the first step we relate the number of state-to-state changes to the angular deviation, and in the second step we use the central limit theorem. Equation (E4) proves Eq. (25) in the main text. As soon as $\wp^{\text {exit }}(t)$ becomes memoryless, that is, $\wp^{\text {exit }}(t) \propto e^{-t /\langle t\rangle^{\text {exit }}}, n_{t}$ becomes Poissonian with mean $\left\langle n_{t}\right\rangle^{M}=t /\langle t\rangle^{\text {exit }}$ and variance $\operatorname{var}\left(n_{t}\right)^{M}=t /\langle t\rangle^{\text {exit }}$, where the superscript " $M$ " signifies the restriction to memoryless Markov jumps.

\section{e. Proof of the upper bound in Fig. 7(f)}

Using Eq. (E4), we obtain the ratio of the true angular mean-squared deviation $\left\langle\delta \theta_{t}^{2}\right\rangle$ and the one deduced from a Markov-jump model that corresponds to setting $\sigma_{\text {exit }}^{M}=\langle t\rangle^{\text {exit }}$, i.e.,

$$
\frac{\left\langle\delta \theta_{t}^{2}\right\rangle}{\left\langle\delta \theta_{t}^{2}\right\rangle^{M}} \simeq \frac{4 \phi_{+} \phi_{-}\left(\langle t\rangle^{\text {exit }}\right)^{2}+\left(\phi_{+}-\phi_{-}\right)^{2} \sigma_{\text {exit }}^{2}}{\left(\langle t\rangle^{\text {exit }}\right)^{2}},
$$

where equality holds as $t \rightarrow \infty$, and the superscript $M$ denotes the Markov-jump limit (see also Ref. [95]). Vanishing extrinsic noise renders the kinetics subMarkovian, $\sigma_{\text {exit }}^{2} \leq\left(\langle t\rangle^{\text {exit }}\right)^{2}$ and immediately yields $\left\langle\delta \theta_{t}^{2}\right\rangle /\left\langle\delta \theta_{t}^{2}\right\rangle^{M} \leq 1$. This implies the dotted line in Fig. 7(f) to be a general upper bound on angular diffusivity.

In this subsection, we show that the splitting probability for the ATPase modeled by a tilted periodic potential is fully determined by the external driving $f$ and is given by Eq. (E3), which notably holds for any $120^{\circ}$ periodic potential. We relate the number of state-to-state transitions to the exit time via the well-established central limit theorem for renewal processes [16] (see also Ref. [37]). We illustrate the forward and backward symmetry of transition time in the mean (see Table VII) and the entire distribution of transition time (see Fig. 21).

In the next section, we provide details about the lower bound on the diffusivity in Fig. 7(f) set by the thermodynamic uncertainty relation (TUR), and in the subsection after that we further address biased diffusion obtained in the limit of vanishing free-energy barriers $(B \rightarrow 0)$.

\section{TUR in periodic systems}

In the previous subsection [see Eq. (E5)], we derive an upper bound on the diffusivity when extrinsic noise vanishes. Conversely, a lower bound on the diffusivity can be deduced from the so-called TUR $[79,80]$. In the limit $t \rightarrow \infty$, the TUR for unicyclic networks implies

$$
\frac{\left\langle\delta \theta_{t}^{2}\right\rangle}{\left\langle\theta_{t}\right\rangle^{2}} \times\left(\phi_{+}-\phi_{-}\right) \frac{t}{\langle t\rangle^{\text {exit }}} \ln \frac{\phi_{+}}{\phi_{-}} \geq 2,
$$

where $\left\langle\theta_{t}\right\rangle / 120^{\circ} \rightarrow\left(\phi_{+}-\phi_{-}\right) t /\langle t\rangle^{\text {exit }}$. Inserting Eqs. (E4) and (E6) into Eq. (E5) yields 


$$
\frac{\left\langle\delta \theta_{t}^{2}\right\rangle}{\left\langle\delta \theta_{t}^{2}\right\rangle^{M}} \geq \frac{2\left(\phi_{+}-\phi_{-}\right)}{\ln \left(\phi_{+} / \phi_{-}\right)}=\frac{2}{f} \frac{e^{f}-1}{e^{f}+1}
$$

where in the last step we define $f \equiv M \times 120^{\circ} /\left(k_{B} T\right)$ and use $e^{f} \equiv \phi_{+} / \phi_{-}$which follows from Eq. (2). The righthand side of the inequality (E7) is depicted in Fig. 7(f) by the solid gray line and coincides with the result for plain biased diffusion (i.e., with the barrier set to zero, $B=0$; see below for more details). This completes the proof that the mean-squared angular deviation (angular diffusivity) in allperiodic one-dimensional systems must lie between the dotted and solid gray lines in Fig. 7(f).

\section{Plain biased diffusion saturates TUR}

Let us finally consider plain biased diffusion, which in the model from Appendix E 3 corresponds to setting $B=0$. Adopting the reduced coordinates $x=\theta / 120^{\circ}$ with $l_{ \pm}=1$ the local potential simplifies to $\beta U_{ \pm}=\mp \beta\left(M \times 120^{\circ}\right) x \equiv$ $\mp f x$. The splitting probability is still given by Eq. (E3). Using Eqs. (S47) and (S50) in the Supplemental Material [75], we obtain the mean and variance of exit time

$$
\begin{aligned}
\langle t\rangle^{\text {exit }} & =\frac{e^{f}-1}{f\left(e^{f}+1\right)}, \\
\sigma_{\text {exit }}^{2} & =\left\langle t^{2}\right\rangle^{\text {exit }}-\left(\langle t\rangle^{\text {exit }}\right)^{2}=\frac{2\left(e^{2 f}-2 f e^{f}-1\right)}{f^{3}\left(e^{f}+1\right)^{2}},
\end{aligned}
$$

respectively, where we further insert the local potential $\beta U_{ \pm}=\mp f x$ along with $D_{ \pm}=1$ into the first line of the corresponding auxiliary integrals in Eq. (9). Inserting Eqs. (E3) and (E8) into Eq. (E5) yields $\left\langle\delta \theta_{t}^{2}\right\rangle /\left\langle\delta \theta_{t}^{2}\right\rangle^{M} \simeq$ $2 f^{-1}\left(e^{f}-1\right)\left(e^{f}+1\right)^{-1}$, which saturates the inequality Eq. (E7).

[1] D. T. Gillespie, Exact Stochastic Simulation of Coupled Chemical Reactions, J. Phys. Chem. 81, 2340 (1977).

[2] D. T. Gillespie, Stochastic Simulation of Chemical Kinetics, Annu. Rev. Phys. Chem. 58, 35 (2007).

[3] R. Elber, D. E. Makarov, and H. Orland, Molecular Kinetics in Condensed Phases: Theory, Simulation, and Analysis (John Wiley \& Sons, Ltd., New York, 2020).

[4] H. H. McAdams and A. Arkin, Stochastic Mechanisms in Gene Expression, Proc. Natl. Acad. Sci. U.S.A. 94, 814 (1997).

[5] J. Paulsson, Models of Stochastic Gene Expression, Phys. Life Rev. 2, 157 (2005).

[6] U. Seifert, Stochastic Thermodynamics, Fluctuation Theorems and Molecular Machines, Rep. Prog. Phys. 75, 126001 (2012).

[7] R. Pastor-Satorras, C. Castellano, P. Van Mieghem, and A. Vespignani, Epidemic Processes in Complex Networks, Rev. Mod. Phys. 87, 925 (2015).
[8] G. R. Bowman and V. S. Pande, Protein Folded States Are Kinetic Hubs, Proc. Natl. Acad. Sci. U.S.A. 107, 10890 (2010).

[9] M. T. Woodside and S. M. Block, Reconstructing Folding Energy Landscapes by Single-Molecule Force Spectroscopy, Annu. Rev. Biophys. 43, 19 (2014).

[10] J. D. Chodera and F. Noé, Markov State Models of Biomolecular Conformational Dynamics, Curr. Opin. Struct. Biol. 25, 135 (2014).

[11] An Introduction to Markov State Models and Their Application to Long Timescale Molecular Simulation, edited by G. R. Bowman, V. S. Pande, and F. Noé (Springer Netherlands, Dordrecht, 2014).

[12] B.E. Husic and V.S. Pande, Markov State Models: From an Art to a Science, J. Am. Chem. Soc. 140, 2386 (2018).

[13] D. J. Wales, M. A. Miller, and T. R. Walsh, Archetypal Energy Landscapes, Nature (London) 394, 758 (1998).

[14] N. Moris, C. Pina, and A. M. Arias, Transition States and Cell Fate Decisions in Epigenetic Landscapes, Nat. Rev. Genet. 17, 693 (2016).

[15] A stochastic waiting time $t$ is said to be memoryless if $\operatorname{Prob}\left[t \geq t_{1}\right]=\operatorname{Prob}\left[t \geq t_{1}+t_{2}\right] / \operatorname{Prob}\left[t \geq t_{2}\right]$ for all $t_{1}$, $t_{2} \geq 0$, which is satisfied if and only if $t$ is exponentially distributed [16].

[16] W. Feller, An Introduction to Probability and Its Applications, 2nd ed. (John Wiley \& Sons, New York, 1971), Vol. II.

[17] K. Neupane, A. P. Manuel, and M. T. Woodside, Protein Folding Trajectories Can Be Described Quantitatively by One-Dimensional Diffusion over Measured Energy Landscapes, Nat. Phys. 12, 700 (2016).

[18] C. Schütte, F. Noé, J. Lu, M. Sarich, and E. VandenEijnden, Markov State Models Based on Milestoning, J. Chem. Phys. 134, 204105 (2011).

[19] G. Hummer, From Transition Paths to Transition States and Rate Coefficients, J. Chem. Phys. 120, 516 (2004).

[20] A. M. Berezhkovskii and D. E. Makarov, SingleMolecule Test for Markovianity of the Dynamics along a Reaction Coordinate, J. Phys. Chem. Lett. 9, 2190 (2018).

[21] D. E. Makarov, Barrier Crossing Dynamics from SingleMolecule Measurements, J. Phys. Chem. B 125, 2467 (2021).

[22] H. S. Chung, K. McHale, J. M. Louis, and W. A. Eaton, Single-Molecule Fluorescence Experiments Determine Protein Folding Transition Path Times, Science 335, 981 (2012).

[23] H. S. Chung and W. A. Eaton, Single-Molecule Fluorescence Probes Dynamics of Barrier Crossing, Nature (London) 502, 685 (2013).

[24] K. Neupane, D. B. Ritchie, H. Yu, D. A. N. Foster, F. Wang, and M. T. Woodside, Transition Path Times for Nucleic Acid Folding Determined from Energy-Landscape Analysis of Single-Molecule Trajectories, Phys. Rev. Lett. 109, 068102 (2012).

[25] D. B. Ritchie and M. T. Woodside, Probing the Structural Dynamics of Proteins and Nucleic Acids with Optical Tweezers, Curr. Opin. Struct. Biol. 34, 43 (2015). 
[26] K. Neupane, D. A. N. Foster, D. R. Dee, H. Yu, F. Wang, and M. T. Woodside, Direct Observation of Transition Paths during the Folding of Proteins and Nucleic Acids, Science 352, 239 (2016).

[27] R. Satija, A. M. Berezhkovskii, and D. E. Makarov, Broad Distributions of Transition-Path Times Are Fingerprints of Multidimensionality of the Underlying Free Energy Landscapes, Proc. Natl. Acad. Sci. U.S.A. 117, 27116 (2020).

[28] J. Gladrow, M. Ribezzi-Crivellari, F. Ritort, and U. F. Keyser, Experimental Evidence of Symmetry Breaking of Transition-Path Times, Nat. Commun. 10, 55 (2019).

[29] J.-Y. Kim and H. S. Chung, Disordered Proteins Follow Diverse Transition Paths as They Fold and Bind to a Partner, Science 368, 1253 (2020).

[30] N. Zijlstra, D. Nettels, R. Satija, D. E. Makarov, and B. Schuler, Transition Path Dynamics of a Dielectric Particle in a Bistable Optical Trap, Phys. Rev. Lett. 125, 146001 (2020).

[31] T. Aquino and M. Dentz, Chemical Continuous Time Random Walks, Phys. Rev. Lett. 119, 230601 (2017).

[32] J. Zhang and T. Zhou, Markovian Approaches to Modeling Intracellular Reaction Processes with Molecular Memory, Proc. Natl. Acad. Sci. U.S.A. 116, 23542 (2019).

[33] R. Zwanzig, Diffusion in a Rough Potential, Proc. Natl. Acad. Sci. U.S.A. 85, 2029 (1988).

[34] W. Thomas, M. Forero, O. Yakovenko, L. Nilsson, P. Vicini, E. Sokurenko, and V. Vogel, Catch-Bond Model Derived from Allostery Explains Force-Activated Bacterial Adhesion, Biophys. J. 90, 753 (2006).

[35] W. E. Thomas, V. Vogel, and E. Sokurenko, Biophysics of Catch Bonds, Annu. Rev. Biophys. 37, 399 (2008).

[36] C. D. Buckley, J. Tan, K. L. Anderson, D. Hanein, N. Volkmann, W. I. Weis, W. J. Nelson, and A. R. Dunn, The Minimal Cadherin-Catenin Complex Binds to Actin Filaments under Force, Science 346, 1254211 (2014).

[37] U. Landman, E. W. Montroll, and M. F. Shlesinger, Random Walks and Generalized Master Equations with Internal Degrees of Freedom, Proc. Natl. Acad. Sci. U.S.A. 74, 430 (1977).

[38] E. W. Montroll and G. H. Weiss, Random Walks on Lattices. II, J. Math. Phys. (N.Y.) 6, 167 (1965).

[39] J. Klafter, A. Blumen, and M. F. Shlesinger, Stochastic Pathway to Anomalous Diffusion, Phys. Rev. A 35, 3081 (1987).

[40] J. Haus and K. Kehr, Diffusion in Regular and Disordered Lattices, Phys. Rep. 150, 263 (1987).

[41] A. K. Faradjian and R. Elber, Computing Time Scales from Reaction Coordinates by Milestoning, J. Chem. Phys. 120, 10880 (2004).

[42] D. Shalloway and A. K. Faradjian, Efficient Computation of the First Passage Time Distribution of the Generalized Master Equation by Steady-State Relaxation, J. Chem. Phys. 124, 054112 (2006).

[43] R. Metzler, J. Klafter, and I. M. Sokolov, Anomalous Transport in External Fields: Continuous Time Random Walks and Fractional Diffusion Equations Extended, Phys. Rev. E 58, 1621 (1998).
[44] E. Barkai, R. Metzler, and J. Klafter, From Continuous Time Random Walks to the Fractional Fokker-Planck Equation, Phys. Rev. E 61, 132 (2000).

[45] I. M. Sokolov and J. Klafter, From Diffusion to Anomalous Diffusion: A Century after Einstein's Brownian Motion, Chaos 15, 026103 (2005).

[46] M. I. Freidlin and A. D. Wentzell, Diffusion Processes on Graphs and the Averaging Principle, Ann. Probab. 21, 2215 (1993).

[47] M. Freidlin and M. Weber, Random Perturbations of Dynamical Systems and Diffusion Processes with Conservation Laws, Probab. Theory Relat. Fields 128, 441 (2004); 137, 595(E) (2006).

[48] M. Hinczewski, Y. von Hansen, J. Dzubiella, and R. R. Netz, How the Diffusivity Profile Reduces the Arbitrariness of Protein Folding Free Energies, J. Chem. Phys. 132, 245103 (2010).

[49] A. Berezhkovskii and A. Szabo, Time Scale Separation Leads to Position-Dependent Diffusion along a Slow Coordinate, J. Chem. Phys. 135, 074108 (2011).

[50] S. Toyabe, T. Watanabe-Nakayama, T. Okamoto, S. Kudo, and E. Muneyuki, Thermodynamic Efficiency and Mechanochemical Coupling of $\mathrm{F}_{1}$-ATPase, Proc. Natl. Acad. Sci. U.S.A. 108, 17951 (2011).

[51] R. Yasuda, H. Noji, M. Yoshida, K. Kinosita, and H. Itoh, Resolution of Distinct Rotational Substeps by Submillisecond Kinetic Analysis of $\mathrm{F}_{1}$-ATPase, Nature (London) 410, 898 (2001).

[52] G. A. Tribello, M. Ceriotti, and M. Parrinello, Using Sketch-Map Coordinates to Analyze and Bias Molecular Dynamics Simulations, Proc. Natl. Acad. Sci. U.S.A. 109, 5196 (2012).

[53] H. Wang and H. Qian, On Detailed Balance and Reversibility of Semi-Markov Processes and SingleMolecule Enzyme Kinetics, J. Math. Phys. (N.Y.) 48, 013303 (2007).

[54] I. A. Martínez, G. Bisker, J. M. Horowitz, and J. M. R. Parrondo, Inferring Broken Detailed Balance in the Absence of Observable Currents, Nat. Commun. 10, 3542 (2019).

[55] A. Puglisi, S. Pigolotti, L. Rondoni, and A. Vupani, Entropy Production and Coarse Graining in Markov Processes, J. Stat. Mech. (2010) P05015.

[56] G. Teza and A. L. Stella, Exact Coarse Graining Preserves Entropy Production Out of Equilibrium, Phys. Rev. Lett. 125, 110601 (2020).

[57] A. M. Berezhkovskii, G. Hummer, and S. M. Bezrukov, Identity of Distributions of Direct Uphill and Downhill Translocation Times for Particles Traversing Membrane Channels, Phys. Rev. Lett. 97, 020601 (2006).

[58] B. W. Zhang, D. Jasnow, and D. M. Zuckerman, Transition-Event Durations in One-Dimensional Activated Processes, J. Chem. Phys. 126, 074504 (2007).

[59] I. Neri, É. Roldán, and F. Jülicher, Statistics of Infima and Stopping Times of Entropy Production and Applications to Active Molecular Processes, Phys. Rev. X 7, 011019 (2017).

[60] I. Neri, É. Roldán, S. Pigolotti, and F. Jülicher, Integral Fluctuation Relations for Entropy Production at Stopping Times, J. Stat. Mech. (2019) 104006. 
[61] I. Neri, Second Law of Thermodynamics at Stopping Times, Phys. Rev. Lett. 124, 040601 (2020).

[62] O. Bénichou and J. Desbois, Exit and Occupation Times for Brownian Motion on Graphs with General Drift and Diffusion Constant, J. Phys. A 42, 015004 (2009).

[63] A. M. Berezhkovskii and A. Szabo, Committors, FirstPassage Times, Fluxes, Markov States, Milestones, and All That, J. Chem. Phys. 150, 054106 (2019).

[64] A. B. Kolomeisky, E. B. Stukalin, and A. A. Popov, Understanding Mechanochemical Coupling in Kinesins Using First-Passage-Time Processes, Phys. Rev. E 71, 031902 (2005).

[65] A. B. Kolomeisky and M. E. Fisher, Molecular Motors: A Theorist's Perspective, Annu. Rev. Phys. Chem. 58, 675 (2007).

[66] H. Kramers, Brownian Motion in a Field of Force and the Diffusion Model of Chemical Reactions, Physica (Amsterdam) 7, 284 (1940).

[67] D. Bartolo, I. Derényi, and A. Ajdari, Dynamic Response of Adhesion Complexes: Beyond the Single-Path Picture, Phys. Rev. E 65, 051910 (2002).

[68] B. T. Marshall, M. Long, J. W. Piper, T. Yago, R. P. McEver, and C. Zhu, Direct Observation of Catch Bonds Involving Cell-Adhesion Molecules, Nature (London) 423, 190 (2003).

[69] P. Cossio, G. Hummer, and A. Szabo, Transition Paths in Single-Molecule Force Spectroscopy, J. Chem. Phys. 148, 123309 (2018).

[70] W. K. Kim and R. R. Netz, The Mean Shape of Transition and First-Passage Paths, J. Chem. Phys. 143, 224108 (2015).

[71] U. Seifert, Stochastic Thermodynamics of Single Enzymes and Molecular Motors, Eur. Phys. J. E 34, 26 (2011).

[72] C. Maes, Local Detailed Balance, SciPost Phys. Lect. Notes 32 (2021).

[73] R. Elber, Milestoning: An Efficient Approach for Atomically Detailed Simulations of Kinetics in Biophysics, Annu. Rev. Biophys. 49, 69 (2020).

[74] A. J. F. Siegert, On the First Passage Time Probability Problem, Phys. Rev. 81, 617 (1951).

[75] See Supplemental Material at http://link.aps.org/ supplemental/10.1103/PhysRevX.11.041047 for the derivation of the main practical result.

[76] P. Hänggi and H. Thomas, Stochastic Processes: Time Evolution, Symmetries and Linear Response, Phys. Rep. 88, 207 (1982).

[77] Y. Klimontovich, Ito, Stratonovich and Kinetic Forms of Stochastic Equations, Physica (Amsterdam) 163A, 515 (1990).

[78] C.-C. Chang, Numerical of Stochastic Differential Equations with Constant Diffusion Coefficients, Math. Comput. 49, 523 (1987).

[79] A. C. Barato and U. Seifert, Thermodynamic Uncertainty Relation for Biomolecular Processes, Phys. Rev. Lett. 114, 158101 (2015).

[80] J. M. Horowitz and T. R. Gingrich, Thermodynamic Uncertainty Relations Constrain Non-Equilibrium Fluctuations, Nat. Phys. 16, 15 (2020).

[81] A. Lapolla, D. Hartich, and A. Godec, Spectral Theory of Fluctuations in Time-Average Statistical Mechanics of
Reversible and Driven Systems, Phys. Rev. Research 2, 043084 (2020).

[82] A. Barato and R. Chetrite, A Formal View on Level 2.5 Large Deviations and Fluctuation Relations, J. Stat. Phys. 160, 1154 (2015).

[83] C. W. Gardiner, Handbook of Stochastic Methods, 3rd ed. (Springer, Berlin, 2004).

[84] Y. He, S. Burov, R. Metzler, and E. Barkai, Random TimeScale Invariant Diffusion and Transport Coefficients, Phys. Rev. Lett. 101, 058101 (2008).

[85] V. Girardin and N. Limnios, On the Entropy for Semi-Markov Processes, J. Appl. Probab. 40, 1060 (2003).

[86] J. Schnakenberg, Network Theory of Microscopic and Macroscopic Behavior of Master Equation Systems, Rev. Mod. Phys. 48, 571 (1976).

[87] C. Jarzynski, Rare Events and the Convergence of Exponentially Averaged Work Values, Phys. Rev. E 73, 046105 (2006).

[88] R. Kawai, J. M. R. Parrondo, and C. Van den Broeck, Dissipation: The Phase-Space Perspective, Phys. Rev. Lett. 98, 080602 (2007).

[89] G. E. Crooks, Nonequilibrium Measurements of Free Energy Differences for Microscopically Reversible Markovian Systems, J. Stat. Phys. 90, 1481 (1998).

[90] U. Seifert, Entropy Production along a Stochastic Trajectory and an Integral Fluctuation Theorem, Phys. Rev. Lett. 95, 040602 (2005).

[91] D. Andrieux, P. Gaspard, S. Ciliberto, N. Garnier, S. Joubaud, and A. Petrosyan, Entropy Production and Time Asymmetry in Nonequilibrium Fluctuations, Phys. Rev. Lett. 98, 150601 (2007).

[92] J. L. Lebowitz and H. Spohn, A Gallavotti-Cohen-Type Symmetry in the Large Deviation Functional for Stochastic Dynamics, J. Stat. Phys. 95, 333 (1999).

[93] P. Gaspard, Fluctuation Theorem for Nonequilibrium Reactions, J. Chem. Phys. 120, 8898 (2004).

[94] M. Esposito and K. Lindenberg, Continuous-Time Random Walk for Open Systems: Fluctuation Theorems and Counting Statistics, Phys. Rev. E 77, 051119 (2008).

[95] C. Maes, K. Netočný, and B. Wynants, Dynamical Fluctuations for Semi-Markov Processes, J. Phys. A 42, 365002 (2009).

[96] D. Andrieux and P. Gaspard, The Fluctuation Theorem for Currents in Semi-Markov Processes, J. Stat. Mech. (2008) P11007.

[97] A. Gomez-Marin, J. M. R. Parrondo, and C. Van den Broeck, Lower Bounds on Dissipation upon Coarse Graining, Phys. Rev. E 78, 011107 (2008).

[98] D.S. Grebenkov and L. Tupikina, Heterogeneous Continuous-Time Random Walks, Phys. Rev. E 97, 012148 (2018).

[99] S.-Z. Yu, Hidden Semi-Markov Models, Artif. Intell. 174, 215 (2010).

[100] M. Esposito, Stochastic Thermodynamics under Coarse Graining, Phys. Rev. E 85, 041125 (2012).

[101] A. T. Hawk and D. E. Makarov, Milestoning with Transition Memory, J. Chem. Phys. 135, 224109 (2011).

[102] A. M. Berezhkovskii and D. E. Makarov, On the Forward/ Backward Symmetry of Transition Path Time Distributions 
in Nonequilibrium Systems, J. Chem. Phys. 151, 065102 (2019).

[103] S. Pigolotti, I. Neri, É. Roldán, and F. Jülicher, Generic Properties of Stochastic Entropy Production, Phys. Rev. Lett. 119, 140604 (2017).

[104] S. Bo, S. H. Lim, and R. Eichhorn, Functionals in Stochastic Thermodynamics: How to Interpret Stochastic Integrals, J. Stat. Mech. (2019) 084005.

[105] P. E. Kloeden, E. Platen, and H. Schurz, Numerical Solution of SDE Through Computer Experiments (Springer, Berlin, 1994).

[106] S. Redner, A Guide to First-Passage Processes (Cambridge University Press, Cambridge, England, 2001).

[107] J. Keilson, A Review of Transient Behavior in Regular Diffusion and Birth-Death Processes, J. Appl. Probab. 1, 247 (1964).

[108] Y. A. Melnikov and M. Y. Melnikov, Green's Functions: Construction and Applications (De Gryter, Berlin, 2012).

[109] D. Hartich and A. Godec, Interlacing Relaxation and First-Passage Phenomena in Reversible Discrete and Continuous Space Markovian Dynamics, J. Stat. Mech. (2019) 024002.

[110] D. Hartich and A. Godec, Duality between Relaxation and First Passage in Reversible Markov Dynamics: Rugged Energy Landscapes Disentangled, New J. Phys. 20, 112002 (2018).
[111] T. R. Gingrich and J. M. Horowitz, Fundamental Bounds on First Passage Time Fluctuations for Currents, Phys. Rev. Lett. 119, 170601 (2017).

[112] J.P. Garrahan, Simple Bounds on Fluctuations and Uncertainty Relations for First-Passage Times of Counting Observables, Phys. Rev. E 95, 032134 (2017).

[113] K. Ptaszyński, First-Passage Times in Renewal and Nonrenewal Systems, Phys. Rev. E 97, 012127 (2018).

[114] K. Proesmans, L. Peliti, and D. Lacoste, in Chemical Kinetics (World Scientific, London, 2019), Chap. 17, pp. 435-453, https://doi.org/10.1142/9781786347015_ 0017.

[115] R. Zwanzig, Diffusion Past an Entropy Barrier, J. Phys. Chem. 96, 3926 (1992).

[116] H. S. Chung, J. M. Louis, and W. A. Eaton, Experimental Determination of Upper Bound for Transition Path Times in Protein Folding from Single-Molecule Photon-by-Photon Trajectories, Proc. Natl. Acad. Sci. U.S.A. 106, 11837 (2009).

[117] D. E. Makarov, Shapes of Dominant Transition Paths from Single-Molecule Force Spectroscopy, J. Chem. Phys. 143, 194103 (2015).

[118] P. Hänggi, P. Talkner, and M. Borkovec, Reaction-Rate Theory: Fifty Years after Kramers, Rev. Mod. Phys. 62, 251 (1990). 